\title{
Flow Structure in a Model of Aircraft Trailing Vortices
}

\author{
Thesis by \\ James M. Faddy \\ In Partial Fulfillment of the Requirements \\ for the Degree of \\ Doctor of Philosophy
}

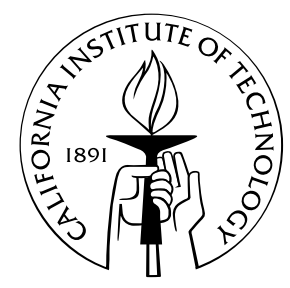

California Institute of Technology

Pasadena, California

2005

(Defended April 29, 2005) 
(c) 2005

James M. Faddy

All Rights Reserved 


\section{Acknowledgements}

First and foremost I would like to thank my friend and supervisor Prof. Dale Pullin. I will never know for sure what you thought when I walked into your office in September 2000 and said that I wanted to learn about vortices. Your enthusiasm, advice, critique

and patience (most of the time) ever since is greatly appreciated and I will truly miss your unique way of thinking.

GALCIT has a fantastic atmosphere, unparalleled anywhere that I have ever been. Thanks for the facilities, the memories and the wine at the Christmas parties! Thanks to Prof. Hornung and the organizers of the Liepmann-Ludwig seminar for allowing me the opportunity to visit Germany as part of this prestigious event.

Much thanks also to my friends and co-workers in the Iris lab, Mike, Phillipe, Ger, Paul, Vincent and Nikoo; without you guys this thesis would surely have taken longer. Special mention to Mike and Phillipe since without me, your theses would surely have been finished sooner!

Finally, Southern California is a truly wonderful place and I must acknowledge some of the greatest friends I will ever make and with whom I shared my time here. Sean, Liz, Dan, Richie, Ilias, Zid and Eimear... I'm sure we'll have a lot to talk about when we're 80 !

This work was supported in part by the National Science Foundation under Grant CTS-0227881. Access to the Hewlett-Packard V2500 computer, located at the California Institute of Technology, was provided by the Center for Advanced Computing Research.

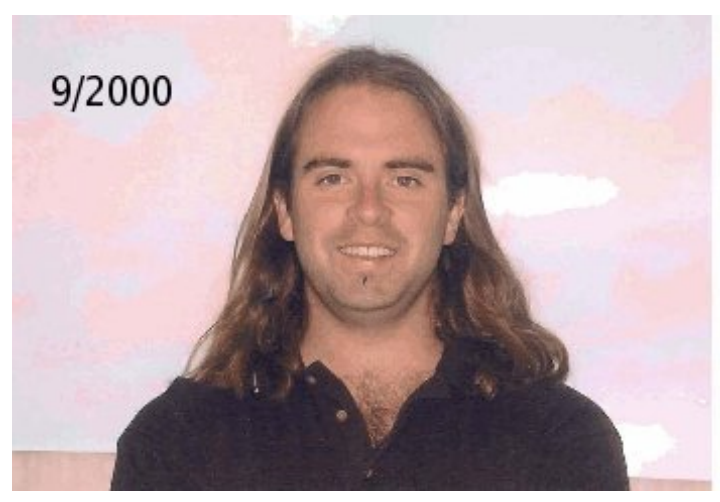




\section{Abstract}

We consider a model of incompressible trailing vortices consisting of an array of counter-rotating structures in a doubly periodic domain, infinite in the vertical direction. The two-dimensional vortex array of Mallier and Maslowe is combined with an axial velocity profile chosen proportional to the initial axial vorticity to provide an initial condition for the vortex wake. This base flow is a weak solution of the three component steady Euler equations in two dimensions thus allowing its linear stability properties to be investigated. These are used to interpret several stages in the development of vortex structure observed in fully three-dimensional DNS at Reynolds numbers $\Gamma /(2 \pi \nu)=\mathcal{O}(1000)$. For sufficiently high axial velocity, its effect can be seen, in that each vortex in the linear array first develops helical structures before undergoing a period of relaminarization. At later times the more slowly growing co-operative elliptical instabilities become apparent; however, the helical structure persists and the observed vortical structures remain coherent for longer periods than in the absence of axial velocity. Using the stretched vortex subgrid model, large-eddy simulation runs are performed at higher Reynolds numbers and a mixing transition identified at about $R e=1-2 \times 10^{4}$. Similar phenomena are observed in these simulations as are seen in the DNS. Next the spatial nature of the true aircraft wake is compared to the temporal approximation commonly employed to simplify the computational complexity of the problem. A model is formulated to acount for the average axial pressure gradients that develops in the spatial wake but is absent from the temporal simulation. The model enables jet- and wake-like axial flows to be distinguished

and the subtle differences in the ensuing turbulent states investigated. Finally, the model is used to investigate co-rotating vortex merger, the new thrust term providing a mechanism to enhance the axial flow further destabilizing the base flow. 


\section{Contents}

Contents $\quad$ iv

List of Figures vii

List of Tables $\quad$ xiv

1 Introduction 1

2 Large Eddy Simulation $\quad 5$

3 Review of the Stretched-Vortex Subgrid Model 11

4 Numerical Method $\quad 17$

4.1 The Pseudospectral Method . . . . . . . . . . . . . . . . 19

4.2 Dealiasing . . . . . . . . . . . . . . . . 20

4.3 Cain's Mapping . . . . . . . . . . . . . . . . . . . . 21

4.4 A Spectral Collocation Method .............. . 23

4.4 .1 Time Integration . . . . . . . . . . . . . . . . . . . . . 24

4.4.2 Poisson/Helmholtz Equations . . . . . . . . . . . . . 24

4.4.3 Parallel Implementation . . . . . . . . . . . . . . . . . . . . . 25

4.5 Test Cases . . . . . . . . . . . . . . . . . . 26

4.5.1 Strained Vortex . . . . . . . . . . . . . . 27

4.5.2 Co-rotating vortex merger . . . . . . . . . . . 28

4.5.3 Axial Velocity in 2D-3C Flows . . . . . . . . . . . . . . . 31 
5 Simulating Trailing Vortices $\quad 37$

5.1 Flow Geometry and Formulation . . . . . . . . . . . . . 37

5.1.1 Initial Condition; $x_{2}^{*} x_{3}^{*}$ plane . . . . . . . . . . . 38

5.1 .2 Axial Velocity . . . . . . . . . . . . . . 39

5.1 .3 Vortex-Induced Strain Rate . . . . . . . . . . . . . . . . . 42

5.1.4 Further Code Verification . . . . . . . . . . . . . . . 43

6 Stability of the M \& M Vortex Array 48

7 3D-3C Direct Numerical Simulation $\quad 54$

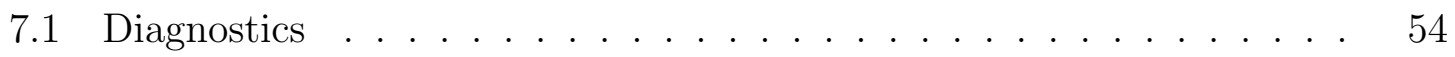

7.2 Direct Numerical Simulation Results . . . . . . . . . . . . . . . 56

7.2.1 Early development ............... 56

7.2 .2 Cooperative instabilities . . . . . . . . . . . . 63

7.2.3 Circulation ...................... 64

7.2 .4 Alignment Statistics _. . . . . . . . . . . . . . . 68

7.2.5 Effect of Resolution and Domain Size . . . . . . . . . . . . . 70

8 Large-Eddy Simulation $\quad 74$

8.1 Flow Evolution Summary . . . . . . . . . . . . . . . . 75

8.2 SGS model performance . . . . . . . . . . . . . . 79

8.3 Early development . . . . . . . . . . . . . . . . . . 80

8.4 Mixing Transition . . . . . . . . . . . . . . . . . . 84

$9 \quad$ Axial Pressure Gradients $\quad 87$

9.1 Modeling Axial Pressure Gradients . . . . . . . . . . . . . . . 91

9.2 Effect on Isolated Vortices . . . . . . . . . . . . . . . . . . 94

9.3 Effect on Co-rotating vortex merger . . . . . . . . . . . . 97

10 Concluding Remarks 103

$\begin{array}{ll}\text { A Point Vortex Induced Strain } & 107\end{array}$ 
B Coefficients for Time Integration 


\section{List of Figures}

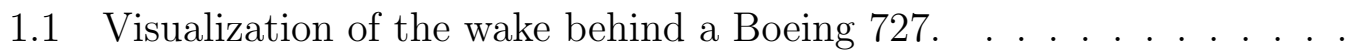

2.1 Idealized energy spectrum showing schematically what portion several common numerical simulation approaches attempt to capture. In green are the years, estimated by Spalart ${ }^{65}$, in which he estimates the simulation of an entire aircraft to be feasible. . . . . . . . . . .

2.2 Turbulent flow velocity component schematic. (a) Physical space: fine scale motions not resolved, their influence is modeled. (b) Spectral space: resolved range, $k<k_{c}$ (cutoff wavenumber $k_{c}$ ), subgrid range

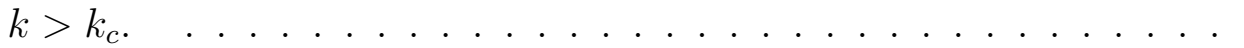

2.3 DNS of 3d Isotropic turbulence performed by Kaneda et al. ${ }^{29}$ on the earth simulator super computer. Visualization is through isosurfaces of vorticity at 3 times the RMS value. . . . . . . . . . . . .

2.4 A typical cell representing the fine scales, showing a single straight, nearly axisymmetric vortex embedded at some orientation. . . . . . 10

3.1 Sketch of the geometry used in the circular average of the structure function relation for the stretched-vortex model. Taken from ${ }^{75}$. . . .

4.1 Contours of vorticity for an initially Gaussian distribution, one transit time later. Top picture is $R e=30$, bottom is $R e=100$.

4.2 Vorticity evolution. Comparison between computation and the theoretical diffusing vortex, $R e=1000$. . . . . . . . . . . . . . . . 29

4.3 Pressure evolution over time for the diffusing vortex. $R e=1000$. . 30 
viii

4.4 Merger of two like signed vortices in an axisymmetric strain rate field. Comparison between the present work(left) and Buntine and Pullin ${ }^{9}$ (right) at $R e=160$. The minimum contour is at $\left|\omega_{s}\right|=1.0$ and the intervals are $\left|\Delta \omega_{s}\right|=9.0$. Times are (from top to bottom) $\mathrm{t}=0,0.05$, $0.1,0.15,0.2 \ldots \ldots \ldots \ldots \ldots \ldots$

4.5 Merger of two like signed vortices in an axisymmetric strain rate field. Comparison between the present work(left) and Buntine and Pullin ${ }^{9}$ (right) at $R e=160$. The minimum contour is at $\left|\omega_{s}\right|=1.0$ and the intervals are $\left|\Delta \omega_{s}\right|=9.0$. Times are (from top to bottom) $\mathrm{t}=0.25,0.3$, $0.35,0.4,0.45$.

4.6 Merger of two like signed vortices in an axisymmetric strain rate field. Comparison between the present work(left) and Buntine and Pullin ${ }^{9}$ (right) at $R e=640$. The minimum contour is at $\left|\omega_{s}\right|=3.0$ and the intervals are $\left|\Delta \omega_{s}\right|=30.0$. Times are (from top to bottom) $\mathrm{t}=0,0.01$, $0.03,0.04,0.05$.

4.7 Merger of two like signed vortices in an axisymmetric strain rate field. Comparison between the present work(left) and Buntine and Pullin ${ }^{9}$ (right) at $R e=640$. The minimum contour is at $\left|\omega_{s}\right|=3.0$ and the intervals are $\left|\Delta \omega_{s}\right|=30.0$. Times are (from top to bottom) $t=0.06$, $0.07,0.08,0.09,0.10$.

4.8 Passive scalar transport during the merger of two like signed vortices at $R e=640$. The initial distribution of the passive scalar is identical to that of vorticity in magnitude however they are of opposite sign. .

4.9 Passive scalar transport during the merger of two like signed vortices at $R e=640$. The initial distribution of the passive scalar is identical to but 90 degrees out of phase with the vorticity. Same sign (top) and opposite sign (bottom). . . . . . . . . . . . . .

5.1 (a) (left) Contours of $\omega_{1}$ and (b) (right) distribution of $u_{3}$ along $x_{3}=0$ for one period of the $\mathrm{M} \& \mathrm{M}$ vortex array with $\mathrm{C}=0.9 . \ldots . .$. 
5.2 Velocity profile through center of vortex array showing definition of the core radius. Left $\mathrm{c}=0.9$ gives $r_{c}=0.506$ and right $\mathrm{c}=0.95$ has $r_{c}=0.33491$.

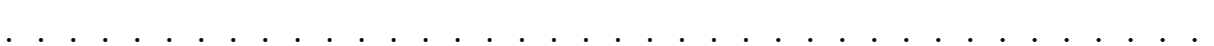

5.3 Profile of axial velocity and $\frac{\partial u}{\partial y}$ through the core of the vortex array for $\mathrm{c}=0.9, \mathrm{q}=1.0 \ldots \ldots \ldots \ldots \ldots \ldots$

5.4 Code validation results of simulations of the Mallier and Maslowe vortex array with superimposed axial velocity. Energy evolution at 3 different $R e_{\Gamma}$ (top left), energy decay rate compared to dissipation (top right), the flow field after one transit time at $R e_{\Gamma}=10^{6}$ (bottom left), and the maximum difference between the flow field after one transit time and the initial condition. . . . . . . . . . . . . .

5.5 Flow visualization of the periodic Crow instability using 1 vorticity isosurface. . . . . . . . . . . . . . . . .

5.6 (a) Effect of grid resolution on energy (top) and enstrophy (bottom). Solid line $=72 \times 256 \times 256$, Squares $=48 \times 128 \times 128$, Circles $=32 \times 96 \times 64$. (b) Perturbation growth rate for the three-dimensional simulation compared with the linear analysis of Julien et al. ${ }^{28}$. . . . . . . .

5.7 Contours of vorticity at the two highest resolutions are layered on top of each other (right) at 3 locations (left) demonstrating grid convergence.

6.1 Growth rate of the anti-symmetric modes (top) with no axial velocity $\left(q_{0}=\infty\right)$, showing the Crow Instability regime at small wavenumbers and the short-wavelength Widnall like modes at high wavenumbers. The symmetric modes (bottom) are similar at high wavenumbers, but are not susceptible to the Crow modes. . . . . . . . . . . . . . 
6.2 Growth rate of the anti-symmetric modes with no axial velocity $\left(q_{0}=\right.$ $\infty)$, showing once again the two regimes. The Crow instability at long wavelengths and the more viscous dependent Widnall instabilities at shorter wavelengths. Filled symbols are taken from the work of Julien et $a l .{ }^{28}$, at $R e=\Gamma / 2 \pi \nu$ of 800 and 10,000 . Open symbols are computed from the present study at $R e=5,000$ and 20,000 . . . . . . . . .

6.3 Growth rate vs axial wavenumber for the anti-symmetric modes of the $\mathrm{M} \& \mathrm{M}$ vortex with $\mathrm{C}=0.9$ for different values of $q_{0} \ldots \ldots \ldots$

7.1 Evolution of volume integrated quantities for the DNS of the $R e=$ $\Gamma / 2 \pi \nu=1000 \mathrm{M} \& \mathrm{M}$ vortex with $C=0.9$ for $q_{0}=1$ (solid lines) and $q_{0}=\infty$ (dashed lines). (a) dissipation and circulation (b) normalized

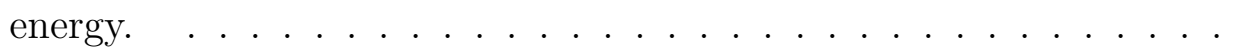

7.2 Flow visualization comparison through an isosurface with $Q=1$ on which contours of dissipation are plotted for the $R e=\Gamma / 2 \pi \nu=1,000$ DNS of the $\mathrm{C}=0.9 \mathrm{M} \& \mathrm{M}$ vortex with $q_{0}=\infty$ left and $q_{0}=1$ right. Evolution is from top to bottom and shown at non-dimensional times (a) 0 (b) 12 (c) 20 (d) 32 (e) 40 (f) 45. . . . . . . . . . .

7.3 Evolution of the maximum axial and azimuthal velocity components as well as the swirl parameter for $R e=\Gamma / 2 \pi \nu=1000$. . . . . . . . 60

7.4 Contours of axial vorticity (left) and azimuthal vorticity (right) colored by axial velocity at time 12 and 20 on slices through one vortex as shown in the top figure. $R e=\Gamma / 2 \pi \nu=1000$. . . . . . . . . . .

7.5 Contours of axial vorticity (left) and azimuthal vorticity (right) colored by axial velocity at time 12 and 20 on slices through one vortex as shown in the top figure. $R e=\Gamma / 2 \pi \nu=3000$. . . . . . . . . . . 62

7.6 Contours of passive scalar for the $R e=\Gamma / 2 \pi \nu=1000, q_{0}=\infty \mathrm{M} \& \mathrm{M}$ vortex at time 45 on slices through the core of the vortex in the xy plane $(\mathrm{a})$ and the yz plane $(\mathrm{b}) . \ldots \ldots \ldots \ldots$ 
7.7 Contours of passive scalar for the $R e=\Gamma / 2 \pi \nu=1000, q_{0}=1 \mathrm{M} \& \mathrm{M}$ vortex at time 45 on slices through the core of the vortex in the xy plane (a) and the yz plane (b). . . . . . . . . . . . . . 66

7.8 Circulation comparison with the experiments of Leweke et al ${ }^{40}$ (Figure 26), with abscissa rescaled to the present non-dimensionalization and shifted to make the onset of decay coincide. Solid circles represent data in a plane where the vortices are brought closer together and open symbols where they are separated. The dashed line is the averaged circulation for the $R e=\Gamma / 2 \pi \nu=1000, q_{0}=\infty$ case. . . . . . . . .

7.9 Joint PDF of the alignment between the vorticity vector and the eigenvectors of $S_{i j}$ for $q_{0}=1$ at (a) $\mathrm{t}=0$, (b) $\mathrm{t}=12$, (c) $\mathrm{t}=20$, (d) $\mathrm{t}=32$, (e) $t=45$ and $(\mathrm{f}) \mathrm{t}=50.20 \times 20$ Bins of equal widths in each direction are used to compute the PDF's. . . . . . . . . . . . . . . 69

$7.10 k_{1}-k_{2}$ spectra in the plane $\mathrm{z}=0$. (a) $q_{0}=1, \mathrm{t}=12$. (b) $q_{0}=0, \mathrm{t}=45 . \quad 71$

7.11 Resolution measurements for the $R e=\Gamma / 2 \pi \nu=1000 \mathrm{M} \& \mathrm{M}$ vortex with $C=0.9$ for $q_{0}=1$ and $q_{0}=\infty$ (a) rate of change of kinetic energy compared to dissipation. (b) relative error between the two. .

8.1 Evolution of dissipation for $R e=\Gamma / 2 \pi \nu=20,000$ with $q_{0}=1$ (solid lines) and $q_{0}=\infty$ (dashed lines). . . . . . . . . . . . . . . 76

8.2 Evolution of (a) energy and (b) circulation for $R e=\Gamma / 2 \pi \nu=20,000$ with $q_{0}=1$ (solid lines) and $q_{0}=\infty$ (dashed lines) . . . . . . . . 77

8.3 Flow visualization comparison through an isosurface with $Q=5$ on which contours of dissipation are plotted for the $R e=\Gamma / 2 \pi \nu=20,000$ LES of the $\mathrm{C}=0.9 \mathrm{M} \& \mathrm{M}$ vortex with $q_{0}=\infty$ left and $q_{0}=1$ right. Evolution is from top to bottom and shown at non-dimensional times (a) 0 (b) 6 (c) 11 (d) 20 (e) 26 (f) 32 .

8.4 Evolution of $\epsilon_{\text {ratio }}$ for $R e=\Gamma / 2 \pi \nu=20,000$ with $q_{0}=1$ (solid lines) and $q_{0}=\infty$ (dashed lines). 
8.5 Effect of $R e$ on the early development of the vortex array with $q_{0}=1$. Dissipation is plotted at Reynolds numbers 1000, 2000, 3000, 4000, 8536, 20,000 (lower curve to upper curve). The first 3 were obtained from DNS and the latter 3 from LES. . . . . . . . . . . . . . .

8.6 Circulation profile for $R e=\Gamma / 2 \pi \nu=20,000$ at $t=10$ (symbols) showing good agreement with the similarity solution of Phillips ${ }^{50}$ (solid line). Also shown are the inner and logarithmic regions predicted by Hoffman and Joubert ${ }^{23}$. . . . . . . . . . . . . . . . .

8.7 Radial distributions of (a) the $\theta$ averaged axial velocity and (b) $\epsilon_{\text {ratio }}$ at several instances in time for $q_{0}=1, R e=\Gamma / 2 \pi \nu=20,000$.

8.8 Dissipation normalized by the maximum swirl velocity and its radial location vs $R e$ at several instances in time.

8.9 Enlarged views of a section of one vortex in the array showing the effect of $R e$ on the flow structure through an isosurface of Q. (a) $R e=$ $\Gamma / 2 \pi \nu=1000, Q=1, t=12$. (b) \& (c) $Q=5, t=6, R e=20,000$ and $R e=200,000$ respectively. (d) \& (e) $Q=5, t=10, R e=20,000$ and $R e=200,000$ respectively. . . . . . . . . . . . . .

9.1 Experimental study performed by de Bruin et al. ${ }^{15}$ on a Fokker airliner model showing additional vortex systems. . . . . . . . . . . . . . . . 88

9.2 Illustration of a device used to generate a counter rotating pair that is essentially 2 dimensional. Figure adapted from $\mathrm{LW}^{40}$. . . . . . . . . 90

9.3 Schematic illustration of the temporal approximation. Axial periodicity is enforced on a small domain with a spatial length scale long enough to capture the turbulent motions. Evolution in time of the periodic vortex on this domain is likened to the spatial evolution of the real wake. . . . . . . . . . . . . . . . . . . . . . . . . . . . . . .

9.4 Comparison of the evolution of the dissipation, between $q=1$ jet(solid lines) and wake-like (dashed lines) axial profiles for the LES of the $R e=8,536 \mathrm{M} \& \mathrm{M}$ vortex with $C=0.9 . \ldots \ldots$ 
xiii

9.5 Radial distributions of the $\theta$ averaged fluctuating components of (a) axial velocity, (b) radial velocity and (c) azimuthal velocity at $\mathrm{t}=10.8$ (squares) and $\mathrm{t}=15.8$ (circles) for the $q=1, R e=8,536 \mathrm{M} \& \mathrm{M}$ initial condition. Solid lines are for a jet like axial flow and dashed lines for

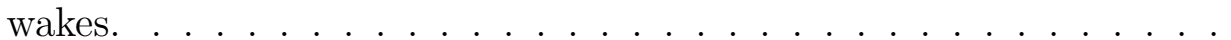

9.6 Evolution of pressure minima for the temporal merger of co-rotating vortices, $R e=1000$. . . . . . . . . . . . . .

9.7 Visualization of co-rotating vortex merger through contours of vorticity on a slice in the plane of the vortex. The initial condition is shown (top) with the visualization plane defined. The flow state at $t=5$ and $t=10$ is shown both with (right) and without (left) the pressure gradient model activated. $R e=100$

9.8 Contours of vorticity on the visualization plane defined in Figure 9.7. The flow state is shown at four times corresponding to $t=15,20,40$ and 80 (top to bottom) both with (right) and without (left) the pressure gradient model activated. $R e=1000 . \quad$. . . . . . . . . . . . . . . 101

9.9 Evolution of the maximum swirl and axial velocity component, as well as the swirl parameter $q$, during the merger of co-rotating vortices both with (bottom) and without (top) the model for axial pressure gradients. $R e=1000$. . . . . . . . . . . . . . . . 102 


\section{List of Tables}

B.1 Coefficients for stiffly-stable schemes . . . . . . . . . . . . . 110 


\section{Chapter 1}

\section{Introduction}

The wake shed by lifting bodies, such as an aircraft wing, rolls up and forms a pair of counter-rotating vortices that can persist back to the original location of the airfoil start-up process (e.g. $\left.{ }^{41}\right)$. In practice, viscous and turbulent diffusion as well as atmospheric turbulence and vortex related instabilities result in decaying strength of wing tip vortices; however, they have been observed to persist tens of miles downstream of large aircraft ${ }^{64}$. These vortices pose a significant risk to following aircraft particularly during take-off and landing where insufficient time may have passed for their decay ${ }^{64}$. An understanding of the underlying physical processes within a turbulent vortex is therefore of pressing practical importance. Studies of coherent structures have shown that many turbulent flows comprise such thin organized vortical structures of various sizes interwined with each other. These observations, coupled with a knowledge of the dynamics of vortical structures and the instabilities arising from their interaction, can aid in the understanding and development of simplified models of the full turbulent flow field.

Kelvin ${ }^{31}$ studied the stability of this flow and showed it to be marginally stable with respect to sinusoidal displacement disturbances; these bending waves simply rotate around the vortex. If the azimuthal component of an imposed external strain rate field balances this self-induced rotation, disturbances will grow exponentially due to the radial component of the strain rate field. When this strain rate is generated by a counter-rotating vortex pair, Crow ${ }^{14}$ showed that the configuration is unstable to 


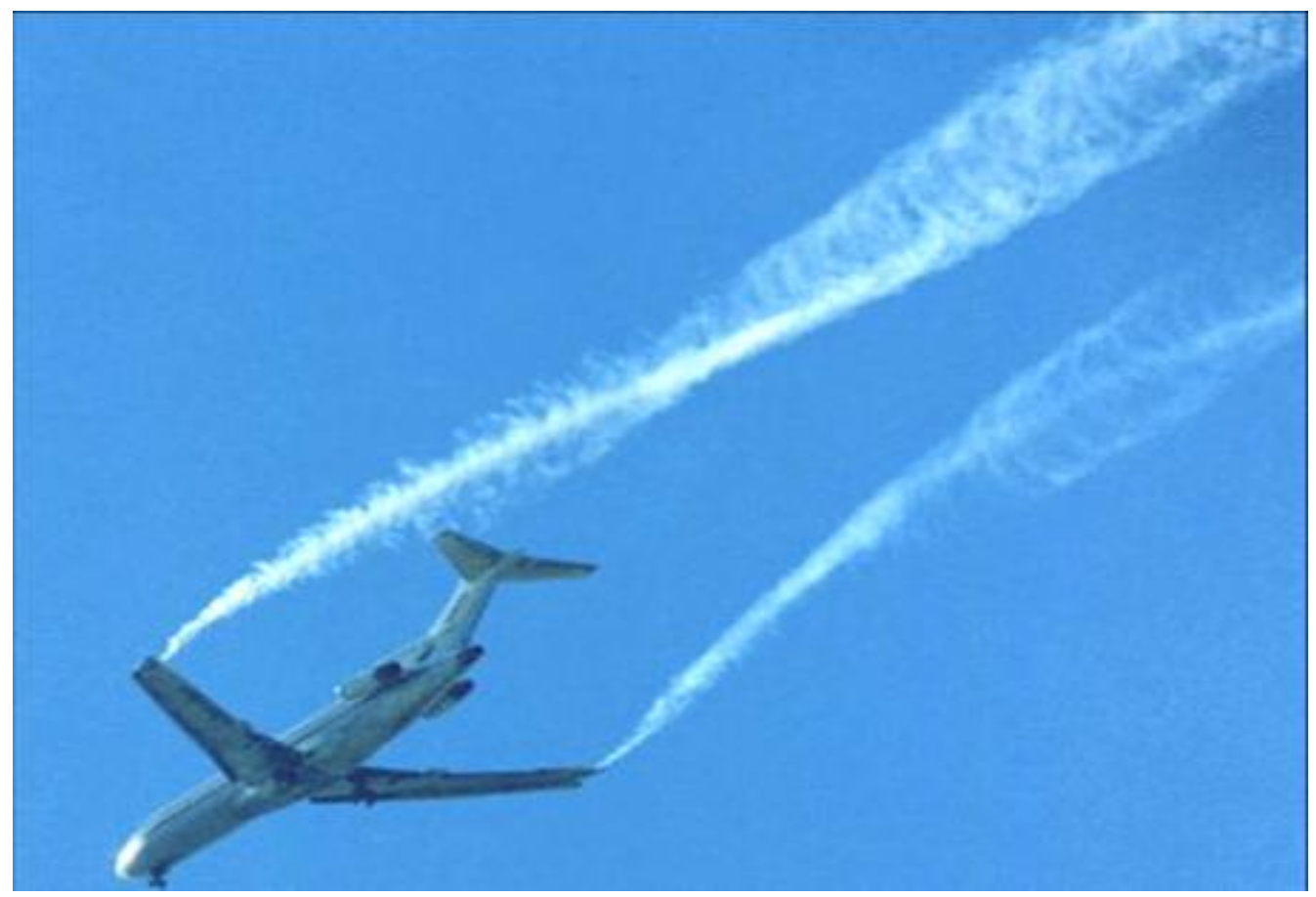

Figure 1.1: Visualization of the wake behind a Boeing 727.

perturbations which are symmetric with respect to the plane separating the vortex; the resulting instability being associated with the lowest Kelvin mode, corresponding to a simple displacement of the entire vortex core. Widnall et al. ${ }^{76}$ identified short wavelength bending instabilities by considering a weakly strained Rankine vortex as a model of the vortex pair. The physical mechanism behind these instability modes is a resonant interaction between the strain rate and Kelvin waves with azimuthal wave numbers $m= \pm 1$ at the same axial frequency. Waves with $m$ of higher order than 1 are axial waves (they do not deflect the vortex axis). Instability modes occurring via an analogous resonant growth of $\mathrm{m}=0$ and $m= \pm 2$ waves have been identified ${ }^{54}$. All of these pair instabilities fall within a more general class of elliptic instabilities that are a result of two dimensional flows with elliptic streamlines being unstable to three-dimensional disturbances ${ }^{52,3}$.

A more realistic vortex has a continuous distribution of vorticity with no clearly defined core. Hoffmann and Joubert ${ }^{23}$ argued that a turbulent vortex is characterized by a central core region of solid-body rotation surrounded by a region in which the 
Reynolds stress dominates and the circulation varies logarithmically with radius. At larger radii, Govindaraju and Saffman ${ }^{22}$ showed that if the vortex grows at a rate faster than that due to molecular diffusion alone, an overshoot in circulation must develop, followed by a long tail of small negative gradient. Utilizing an inviscid theory for the roll-up of a vortex sheet, Moore and Saffman ${ }^{48}$ analyzed the internal structure of a laminar trailing vortex, showing that an axial velocity must be present whose magnitude and direction depend upon the wing tip loading. The effect that jet- and wake-like axial velocity profiles have on the development of a turbulent line vortex was studied experimentally by Phillips and Graham ${ }^{51}$. They found that imposing an axisymmetric jet or a wake resulted in greater turbulence intensities and Reynolds shear stresses within the vortex, the former having the stronger effect.

The Lamb-Oseen vortex (LOV henceforth), defined by a vorticity distribution that decreases exponentially with the square of the radius, represents the flow field that results from a decaying line vortex and is often chosen as a physically realistic base flow for analyzing trailing vortices. In cylindrical coordinates, the LOV is defined by an azimuthal velocity $u_{\theta}^{*}$ that varies with radius $r^{*}$ as

$$
u_{\theta}^{*}\left(r^{*}\right)=\frac{\Gamma^{*}}{2 \pi r^{*}}\left(1-e^{\left(r^{*} / \delta^{*}\right)^{2}}\right)
$$

where $\Gamma^{*}$ is the vortex circulation, and $\delta^{*}$ some length scale $(2 \sqrt{\nu t}$ for the self similarly decaying line vortex). Here and henceforth the '*' superscript indicates dimensional quantities. A convenient Reynolds number is then defined as $R e=\Gamma^{*} / 2 \pi \nu^{*}$. This flow is Rayleigh stable. A strain rate field produced by an axial velocity proportional to the vorticity is sufficient to render the flow linearly unstable ${ }^{36}$ for small values of the swirl parameter (denoted by $q$ ) defined in terms of the relative strength of the swirling velocity to the axial flow

$$
u_{x}^{*}(r)=u_{d}^{*} e^{-\left(r^{*} / \delta^{*}\right)^{2}}, \quad q=\frac{\Gamma^{*}}{2 \pi \delta^{*} u_{d}^{*}} .
$$

Direct numerical simulation (DNS) and Large-eddy simulation (LES) ${ }^{66,67,25}$ of the 
resulting flow, termed the Batchelor vortex (BV henceforth), have shown that while the base flow is linearly unstable, non-linear interactions drive the flow to a stable configuration through a weakening of the axial flow. As $q$ increases, disturbances in the core of the vortex are damped and the rotation of the core ultimately results in a relaminarization. Such simulations typically employ the temporal approximation which aims to represent the spatial evolution of the flow by the transient development of a finite length segment of the domain. Imposing periodicity in the axial direction then results in a realizable simulation. In most implementations this eliminates, from the axial momentum equation, the influence of any mean pressure gradients that may develop in the vortex core. Consequently this decoupling of the pressure gradient prevents the distinction between a jet and a wake. Another shortcoming of simulating an isolated BV is that it neglects the straining influence of the counter-rotating pair and hence the ensuing cooperative instabilities discussed previously.

We consider the interaction of an array of counter-rotating structures as a model for the trailing vortex pair consequent of a lifting body such as an airfoil. In Chapter 4, we detail the formulation and validation of a specialized code for performing simulations on a domain with two periodic directions, unbounded in the third. In Chapter 5 , we define a family of initial conditions that satisfy the 2D-3C Euler equations on this domain, allowing its linear stability properties to be studied. Here and subsequently we use 2D-3C to denote a flow with three velocity components that depend on two spatial dimensions, as distinguished from 3D-3C in which the flow depends on all three spatial dimensions. The linear stability of this base flow is investigated in Chapter 6 and used to interpret in Chapters 7 and 8 the structures that result from full 3D-3C viscous DNS and LES of the evolution of the array. The study of trailing vortices is completed in Chapter 9 by considering the effect of axial pressure gradients that occur in the true spatial development of the wake but which are absent when the temporal approximation is made to simplify the numerics. A model for incorporating the effect is determined and its influence on isolated as well as co-rotating vortices is studied. We now present an introduction to Large Eddy Simulation, before detailing the specifics of the Stretched Spiral Vortex Subgrid-stress model in Chapter 3. 


\section{Chapter 2}

\section{Large Eddy Simulation}

To motivate Large Eddy Simulation (LES) ${ }^{61}$ and the role it plays in modeling turbulent phenomena, consider the energy spectrum schematic shown in Figure 2.1. A Direct Numerical Simulation (DNS), by definition, has to capture all scales of motion right down to the smallest scales. An estimate for what this entails can be made by considering the ratio of the largest eddies in the flow of size $l$, to the smallest motions that are dissipated at the Kolmogorov scale $\eta=\left(\nu^{3} / \epsilon\right)^{1 / 4}$ (cf. Ref. ${ }^{73}$ ). Here, $\nu$ is the viscosity and $\epsilon$ the dissipation which we assume ${ }^{72}$ to occur at the same rate at which energy is supplied from the large scales: $\epsilon \propto u^{3} / l$. The number of grid points in one dimension is thus of the order $l / \eta \propto R e^{3 / 4}$. For a 3D simulation the time step depends on the mesh width, and hence the total computational cost scales with $R e^{3}$. At present such simulations are too computationally intensive for all but the most simple of flows. On the other hand the Reynolds Averaged Navier Stokes technique $(\text { RANS })^{57}$, is widely used in industrial applications where only some averaged effect of the flow is of interest. Both RANS and its unsteady counterpart, URANS ${ }^{6,17}$, capture only a very small fraction of the total energy and rely on phenomenologically-based arguments to model the averaged effect of the remaining motions. Neither of these approaches converge to the DNS as the mesh is refined since the turbulence models have no knowledge of the grid size. The Partially Averaged Navier Stokes (PANS) formulation is a technique that is currently being developed ${ }^{21}$ to bridge this gap by introducing a spatially-varying blending parameter governing the portion of the 
(U)RANS turbulence model that is used; as the parameter approaches zero, a DNS is attempted.

LES, as its name suggests, captures the large energy carrying motions in the flow, while modeling only the small scales; the hope being that these are less flow dependent and allow a general model to be constructed. There are several schools of thought on LES $^{53}$, depending on whether it is viewed as a physical model ${ }^{58}$ or a numerical procedure $^{5}$. In the first of these, the subgrid model is constructed in such a way that DNS is approached as the mesh is refined towards the Kolmogorov length scale, whereas the latter carefully chooses a numerical method such that the numerical error behaves as the model.

Spalart ${ }^{65}$ conducted a survey of these techniques for turbulence modeling and compared their feasibility by considering the simulation of an entire aircraft. He concluded that while RANS and URANS were already deemed achievable for this problem, LES would remain too computationally intensive for another 45 years, DNS lagging by a further 35 years.

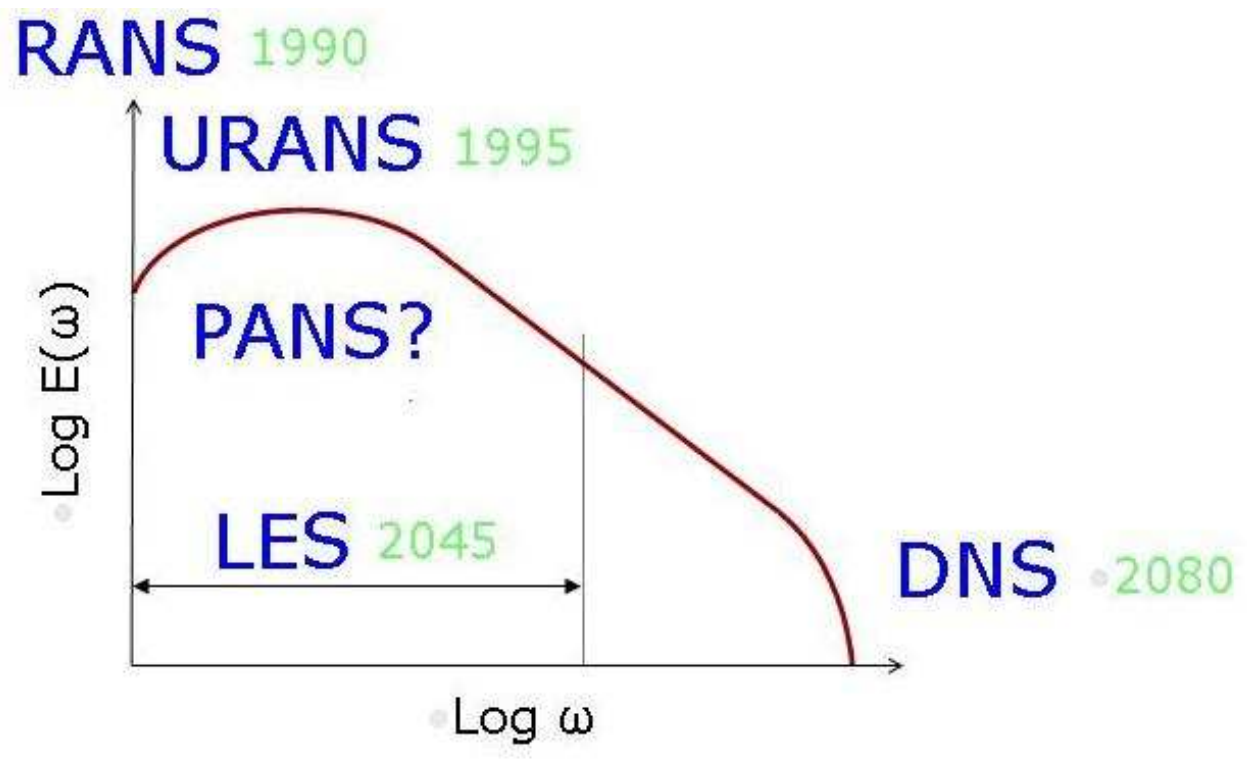

Figure 2.1: Idealized energy spectrum showing schematically what portion several common numerical simulation approaches attempt to capture. In green are the years, estimated by Spalart ${ }^{65}$, in which he estimates the simulation of an entire aircraft to be feasible. 
The starting point for LES is the resolved scale Navier-Stokes equations* (N-S henceforth). In the current work, we consider the case of incompressible, constant density flow and thus

$$
\begin{gathered}
\frac{\partial \tilde{U}_{i}}{\partial x_{i}}=0 \\
\frac{\partial \tilde{U}_{i}}{\partial t}+\frac{\partial}{\partial x_{j}}\left(\tilde{U}_{i} \tilde{U}_{j}\right)=-\frac{1}{\rho} \frac{\partial \tilde{P}}{\partial x_{i}}+\frac{\partial \tau_{i j}}{\partial x_{j}}+\nu \frac{\partial^{2} \tilde{U}_{i}}{\partial x_{j} \partial x_{j}},
\end{gathered}
$$

where $\tilde{U}_{i}$ and $\tilde{P}$ are the filtered scale velocity and pressure fields respectively, and $\tau_{i j}$ is the subgrid-stress (SGS) tensor which represents the effect of the subgrid scales on the resolved flow. These can be derived formally by applying a filter to the full N-S equations ${ }^{37}$ and hence

$$
\tau_{i j}=\tilde{U}_{i} \tilde{U}_{j}-\widetilde{U_{i} U_{j}}
$$

Figure 2.2 shows schematically what the DNS and LES evolution of some flow field might look like. The LES captures the large scale motions while modeling the small scales. It is important to note that the filtering operation cannot be performed explicitly in actual LES, and so while the red line represents what a DNS flow field might resemble, the resolved flow (shown schematically in green) is the result of the numerical integration of 2.2 using a given numerical method on a grid whose smallest scale is much larger than the smallest turbulence scale. Hence, even if the initial condition for a large-eddy simulation is the filtered DNS field, the link between the two at any point later in time can only be statistical ${ }^{53}$.

There are many LES models; however, they can broadly be classified as belonging to one of three groups. The first group uses phenomenological arguments to determine the subgrid contribution. The Smagorinsky model ${ }^{63}$ belongs to this group, and uses the local grid size and the resolved rate of strain tensor to determine an eddy viscosity.

$$
\tau_{i j}-\frac{1}{3} \delta_{i j} \tau_{k k}=-2(C \Delta)^{2}|\tilde{S}| \tilde{S_{i j}}
$$

${ }^{*}$ Here we use the index notation $\left(U_{1}, U_{2}, U_{3}\right)$ for the velocity components and $\left(x_{1}, x_{2}, x_{3}\right)$ for coordinates in space. We will use $(U, V, W)$ and $(x, y, z)$ interchangeably if it simplifies notation. 


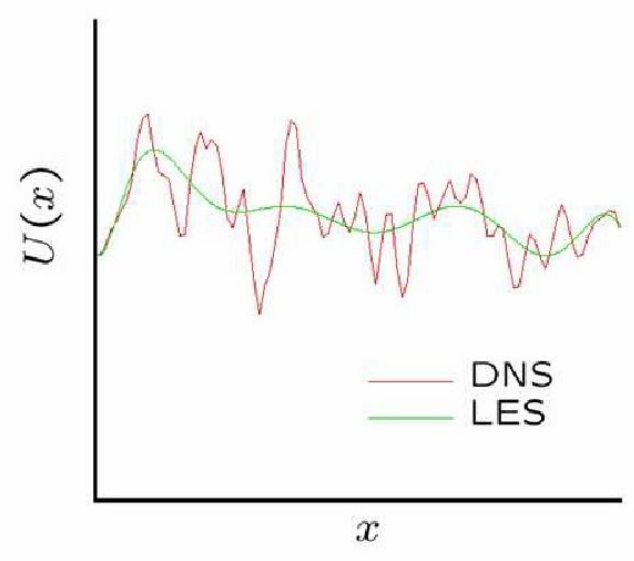

(a)

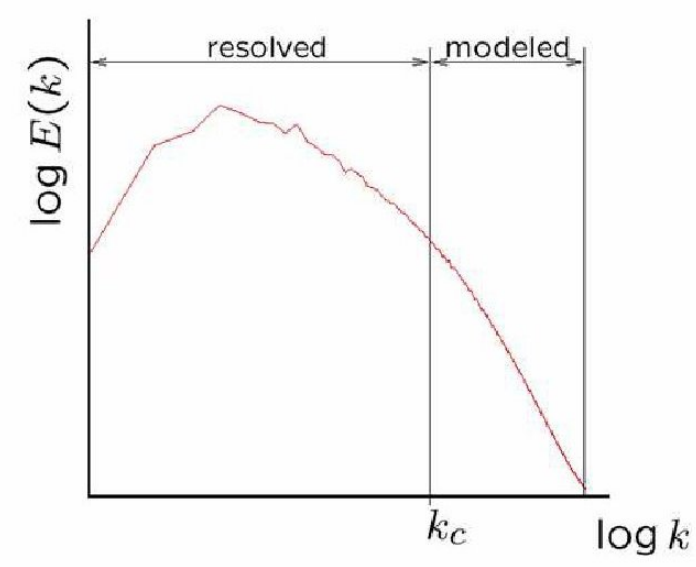

(b)

Figure 2.2: Turbulent flow velocity component schematic. (a) Physical space: fine scale motions not resolved, their influence is modeled. (b) Spectral space: resolved range, $k<k_{c}$ (cutoff wavenumber $k_{c}$ ), subgrid range $k>k_{c}$.

In its dynamic counterpart, an explicit filter along with the Germano Identity is used to determine the Smagorinsky constant $C^{19,20}$. The second group of models, which contains the approximate deconvolution algorithm ${ }^{37}$, attempts to explicitly invert the LES filter. Finally, structure based models, as their name suggests, assume some form for the subgrid structure and compute subgrid contributions based on their properties.

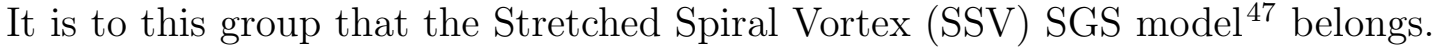

The motivation for structure-based modeling comes from experimental and computational evidence that turbulent flow fields comprise coherent structures ${ }^{62,1,27}$. The simplifying approximation of 'box turbulence' allows numerical simulations to resolve the highest possible Reynolds number. These intense vorticity structures with one dimension significantly longer than the others (worms) have been found to have a strong tendency to point in the direction of the most extensional eigenvector of the rate of strain tensor ${ }^{1}$. Figure 2.3 shows a portion of the flow field from the largest ever such DNS computed to date. Performed by Kaneda et al. ${ }^{29}$ on the Earth Simulator supercomputer, it uses $4096^{3}$ grid points and took 43 hours at 16.4 T-flops to compute one eddy turn-over time. Flow visualization is through an isosurface of vorticity at a magnitude significantly higher than the RMS value, showing the tube like vortex 


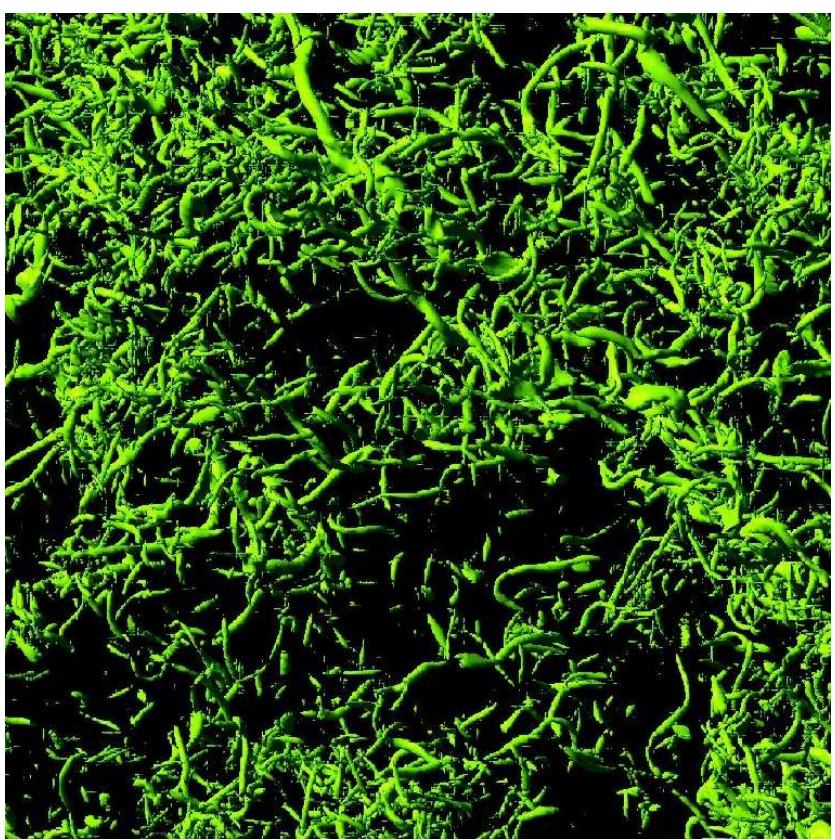

Figure 2.3: DNS of 3d Isotropic turbulence performed by Kaneda et al. ${ }^{29}$ on the earth simulator super computer. Visualization is through isosurfaces of vorticity at 3 times the RMS value.

structures that exist at the fine scales. It is postulated that such highly vortical small scale structures convect the lower vorticity structures into spirals, hence motivating the stretched spiral vortex model.

Figure 2.4 shows that in the SSV model the subgrid at each collocation point is assumed to comprise a single straight, nearly axisymmetric vortex aligned with some orientation determined dynamically by local properties of the resolved flow (these can be viewed as being similar to the tubular vortices seen in DNS). The Lundgren stretched spiral vortex ${ }^{42}$ is chosen to describe the subgrid structure since it is an approximate solution to the Navier Stokes equations exhibiting the attractive property of a $-5 / 3$ inertial range. In its initial form, Misra and Pullin ${ }^{47}$ utilized this idea and estimated model parameters by first assuming a local balance between the total dissipation on one side, and the sum of the resolved-scale dissipation and the subgrid dissipation on the other. The Kolmogorov prefactor was either held constant for the simulation, or else a continuity condition between the resolved and subgrid 
energy spectra used to obtain a global system of equations that could be solved, allowing the SGS tensor to be computed from the resolved scale quantities without specifying a Kolmogorov constant. Later, Voelkl and Pullin ${ }^{75}$ formulated a localized approach, estimating the model parameters using the relationship between the 2D structure functions and the one dimensional energy spectrum tensor to match the resolved field to the subgrid contribution. It should be noted that in the current form, we do not assume explicitly a form of the energy spectrum in computing the subgrid contributions; rather, we use model structures that exhibit the desired form. This allows for anisotropy in the subgrid and for modeling of quantities other than the stress, e.g., passive scalar transport. A detailed derivation of the model is given in Chapter 3; however, at this point we must stress that the model is not of the eddy viscosity type, and no explicit filtering is used.
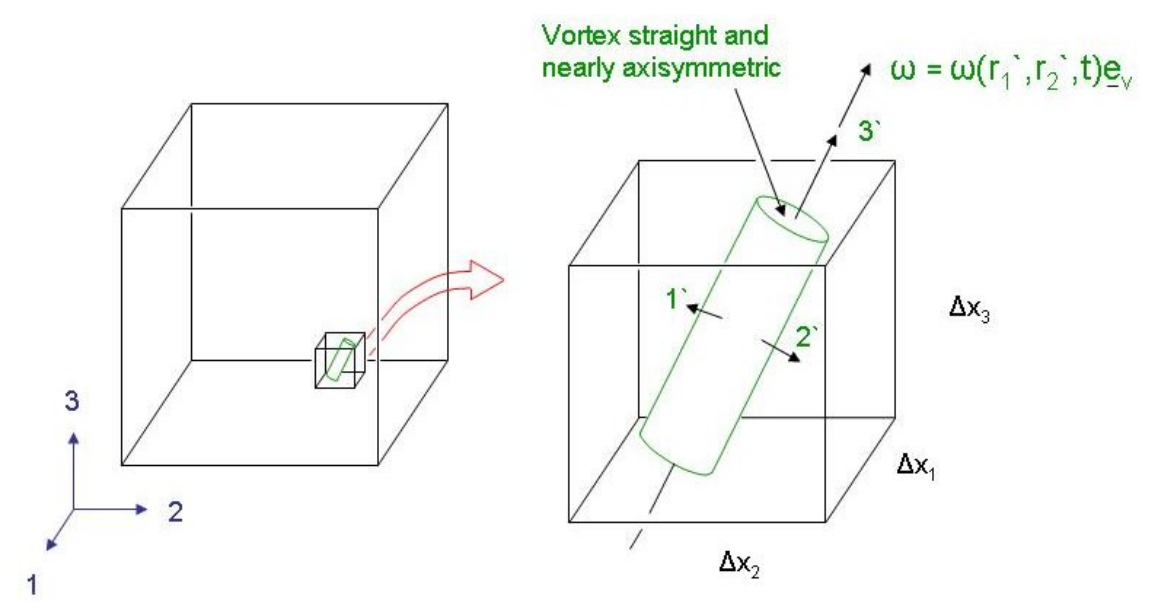

Figure 2.4: A typical cell representing the fine scales, showing a single straight, nearly axisymmetric vortex embedded at some orientation.

The SSV SGS model has enjoyed considerable success, particularly for unbounded flows, such as decaying isotropic turbulence ${ }^{47}$ and compressible isotropic turbulence ${ }^{35}$, but also for the logarithmic and core region of channel flow ${ }^{74}$ (DNS resolution being required in the viscous sublayer). This research focuses on extending the application of the model to another unbounded flow, the trailing vortex. 


\section{Chapter 3}

\section{Review of the Stretched-Vortex Subgrid Model}

Experimental studies of turbulent flow fields suggest that the fine scale structure consists of vortex tubes. This motivates the development of models for turbulence quantities that assume that the small scale vorticity can be described by ensembles of coherent laminar vortex structures. Assuming these elements are straight and nearly axisymmetric, and ignoring finite length effects as well as axial flow, Pullin and Saffman ${ }^{55}$ derived the resulting two point velocity correlation tensor for homogeneous anisotropic turbulence. If the unresolved scales in a LES are assumed to be discrete realizations of such a flow, the subgrid stress tensor can be modeled as

$$
\tau_{i j}=2 \int_{k_{c}}^{\infty} E(k) d k\left\langle E_{p i} Z_{p q} E_{q j}\right\rangle
$$

where $k_{c}$ is the low wavelength cutoff, $E(k)$ the subgrid energy spectrum, $E_{i j}$ the transformation from vortex fixed coordinates, and $Z_{i j}$ a diagonal matrix with diagonal elements $\left(\frac{1}{2}, \frac{1}{2}, 0\right)$. The angle brackets in (3.1) denote the expectation of a function averaged over all possible orientations of the vortex structures. Misra and Pullin ${ }^{47}$ used a probability density function (PDF) governing the orientation over the Euler angles $\alpha$ and $\beta$ (assuming independence of the spin angle) given by a linear 
combination of the product of delta functions

$$
\hat{P}(\alpha, \beta)=4 \pi \sum_{e} \lambda_{e} \delta\left(\beta-\beta_{e}\right) \delta\left(\cos \alpha-\cos \alpha_{e}\right)
$$

where $\lambda$ is a weighting governing the likelihood that the structure is aligned with the Euler angles $\alpha_{c}$ and $\beta_{e}$. Naturally,

$$
\frac{1}{4 \pi} \int_{0}^{2 \pi} \int_{0}^{\pi} \hat{P}(\alpha, \beta) \sin (\alpha) d \alpha d \beta=\sum_{e} \lambda_{e}=1 .
$$

Defining the unit vector describing alignment with $\alpha_{e}, \beta_{e}$ by $e^{e}$, whose components are

$$
e_{1}^{e}=\sin \left(\alpha_{e}\right) \cos \left(\beta_{e}\right), \quad e_{2}^{e}=\sin \left(\alpha_{e}\right) \sin \left(\beta_{e}\right), \quad e_{3}^{e}=\sin \left(\alpha_{e}\right),
$$

Equation 3.1 can be written as

$$
\tau_{i j}=\sum_{e} \lambda_{e} \int_{k_{c}}^{\infty} E\left(k, \alpha_{e}, \beta_{e}\right)\left(\delta_{i j}-e_{i}^{e} e_{j}^{e}\right) d k
$$

To estimate this, Misra and Pullin ${ }^{47}$ assume a Kolmogorov form of $E(k)$ with a sharp viscous cut-off

$$
E(k)=\mathcal{K}_{0} \epsilon^{2 / 3} k^{-5 / 3}, \quad k_{c}<k<J \eta^{-1}=0, k>J \eta^{-1},
$$

where $\mathcal{K}_{0}$ is the Kolmogorov prefactor, $\eta=\left(\nu^{3} / \epsilon\right)^{1 / 4}$ is the local Kolmogorov length associated with the local subgrid realization of homogeneous anisotropic turbulence, and $J$ a cutoff parameter.

In deriving the physical-space version of the Stretched-Vortex model, Pullin and Voelkl ${ }^{74}$ use the relationship between the energy spectrum in Fourier space and the second order structure functions in physical space. Here we follow their analysis in outlining the principal features of the SSV SGS model while retaining the possibility of multiple alignment. The starting point is the trace of the one-dimensional spectrum 
tensor $\Theta$,

$$
\Theta_{i i}=\frac{1}{2 \pi^{2}} \int_{0}^{\pi} \int_{0}^{2 \pi} \int_{|k 3 / \sin \alpha|}^{\infty} E(k, \alpha, \beta)\left(k^{2}-\frac{k_{3}^{2}}{\sin ^{2} \alpha}\right)^{-\frac{1}{2}} \hat{P}(\alpha, \beta) d \alpha d \beta d k
$$

After substitution of the PDF, the integration over the Euler angles can be carried out giving

$$
\Theta_{i i}=\frac{2}{\pi} \sum_{e} \int_{\left|k_{3} / \sin \alpha_{e}\right|}^{\infty} E\left(k, \alpha_{e}, \beta_{e}\right)\left(k^{2}-\frac{k_{3}^{2}}{\sin ^{2} \alpha_{e}}\right)^{-\frac{1}{2}} \frac{\lambda_{e}}{\sin \alpha_{e}} d k
$$

Performing a one-dimensional Fourier transform, an expression for the trace of the velocity correlation tensor for a separation $(0,0, r)$ is obtained

$R_{i i}(0,0, r)=\frac{2}{\pi} \int_{k_{3}=-\infty}^{\infty} \sum_{e} \int_{k=\left|k_{3} / \sin \alpha_{e}\right|}^{\infty} E\left(k, \alpha_{e}, \beta_{e}\right)\left(k^{2}-\frac{k_{3}^{2}}{\sin ^{2} \alpha_{e}}\right)^{-\frac{1}{2}} \frac{\lambda_{e}}{\sin \alpha_{e}} d k e^{i k_{3} r} d k_{3}$,

or, changing the order of integration

$R_{i i}(0,0, r)=\frac{2}{\pi} \sum_{e} \frac{\lambda_{e}}{\sin \alpha_{e}} \int_{k=0}^{\infty} E\left(k, \alpha_{e}, \beta_{e}\right) \int_{k_{3}=-k \sin \alpha_{e}}^{k_{3}=k \sin \alpha_{e}}\left(k^{2}-\frac{k_{3}^{2}}{\sin ^{2} \alpha_{e}}\right)^{-\frac{1}{2}} e^{i k_{3} r} d k_{3} d k$.

By using the substitution $s=k_{3} / k \sin \alpha_{e}$, the expression can be simplified

$$
R_{i i}(0,0, r)=\frac{2}{\pi} \int_{k=0}^{\infty} \sum_{e} \lambda_{e} E\left(k, \alpha_{e}, \beta_{e}\right) \int_{s=-1}^{1}\left(1-s^{2}\right)^{-1 / 2} e^{i k s r \sin \alpha_{e}} d s d k
$$

and the inner integration carried out analytically with the aid of the zeroth-order Bessel function of the first kind $\left(J_{0}\right)$, giving

$$
R_{i i}(0,0, r)=2 \int_{k=0}^{\infty} \sum_{e} \lambda_{e} E\left(k, \alpha_{e}, \beta_{e}\right) J_{0}\left(r k \sin \alpha_{e}\right) d k .
$$

Now $R_{i j}=\overline{U_{i} \hat{U}_{j}}$ where $U_{i}$ and $\hat{U}_{i}$ are velocity components at points with coordinates $\mathrm{x}$ and $\hat{\mathbf{x}}=\mathbf{x}+r \mathbf{e}_{3}$, respectively and where the overline represents a volume average. 
Hence

$R_{i i}=\overline{U_{1}^{2}}+\overline{U_{2}^{2}}+\overline{U_{3}^{2}}-\frac{1}{2}\left[\overline{\left(\hat{U}_{1}-U_{1}\right)^{2}}+\overline{\left(\hat{U}_{2}-U_{2}\right)^{2}}+\overline{\left(\hat{U}_{2}-U_{2}\right)^{2}}\right]=2 \int_{0}^{\infty} E(k) d k-\frac{1}{2} \overline{\overline{|\hat{U}-U|^{2}}}$.

This can be related to the structure function by

$$
R_{i i}=2 \int_{0}^{\infty} \sum_{e} \lambda_{e} E\left(k, \alpha_{e}, \beta_{e}\right) d k-\frac{1}{2} \overline{\overline{|\hat{U}-U|^{2}}}
$$

where $\hat{U}=U\left(\mathbf{x}+r \mathbf{i}_{\mathbf{3}}\right)$. Hence

$$
F_{2}(r ; \mathbf{x})=4 \int_{k=0}^{\infty} \sum_{e} \lambda_{e} E\left(k, \alpha_{e}, \beta_{e}\right)\left[1-J_{0}\left(r k \sin \alpha_{e}\right)\right] d k .
$$

For application to LES the structure function of the true velocity field is split into a resolved-scale contribution, $\hat{F}_{2}(r ; \mathbf{x})=\overline{\overline{|\hat{U}-U|^{2}}}$ and a subgrid component $F_{2, s g s}$, where

$$
F_{2}(r ; \mathbf{x})=\hat{F}_{2}(r ; \mathbf{x})+F_{2, s g s}(r ; \mathbf{x}) .
$$

From this an expression for the resolved scale structure function is obtained

$$
\hat{F}_{2}(r ; \mathbf{x})=4 \sum_{e} \lambda_{e} \int_{k=0}^{k_{c}} E\left(k, \alpha_{e}, \beta_{e}\right)\left[1-J_{0}\left(r k \sin \alpha_{e}\right)\right] d k .
$$

While this expression involves integrating the unknown energy spectrum over the resolved scales, it is important to note that the term involving the Bessel function $1-J_{0}(x)$ tends to $x^{2}$ as $x$ goes to zero, and hence the dominant contribution comes from larger wavenumbers. This, along with the assumption that the grid scale cut-off in LES is in the inertial range of the energy spectrum, away from the viscous cutoff suggests that a Kolmogorov form of $E(k)$ should be adequate for the purpose of evaluating the integral. Hence upon substitution of $E(k)=\kappa_{0} \epsilon^{2 / 3} k^{-5 / 3}$ into the above equation and subsequent manipulation, an equation for the unknown group $\kappa_{0} \epsilon^{2 / 3}$ in 
terms of resolved scale quantities is obtained.

$$
\kappa_{0} \epsilon^{2 / 3}=\frac{\hat{F}_{2}(r ; \mathbf{x})}{4 \sum_{e} \lambda_{e} \int_{k=0}^{k_{c}} k^{-5 / 3}\left[1-J_{0}\left(r k \sin \alpha_{e}\right)\right] d k} .
$$

In order to evaluate the resolved-scale structure function and hence obtain an estimate of subgrid parameters at each grid point a circular average over a radius $r$ is used as shown in Figure 3.1. A circular average was chosen in previous work ${ }^{74}$ as it is equally

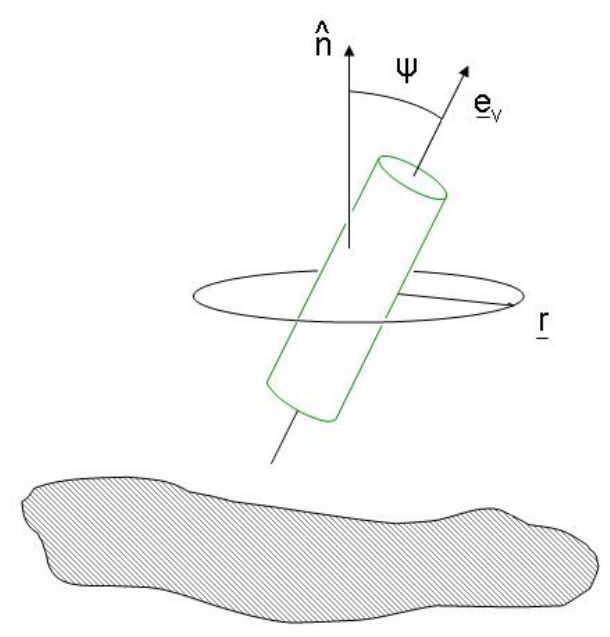

Figure 3.1: Sketch of the geometry used in the circular average of the structure function relation for the stretched-vortex model. Taken from ${ }^{75}$.

applicable to free and wall-bounded flows whereas a spherical average is unsuitable for the latter. Defining the circular averaged structure function by $\hat{F}_{2}^{\odot}$, and using the change of variable $s=k \Delta, \Delta$ being the length scale of the cut-off $\left(k_{c}=\pi / \Delta\right)$, the 
expression becomes

$$
\kappa_{0} \epsilon^{2 / 3}=\frac{\pi \hat{F}_{2}^{\odot}(r ; x)}{2 \Delta^{2 / 3} \sum_{e} \lambda_{e} \int_{\phi=0}^{2 \pi} \int_{s=0}^{\pi} s^{-5 / 3}\left[1-J_{0}\left(s(r / \Delta) \sqrt{1-\sin ^{2} \psi_{e} \cos ^{2} \phi}\right)\right] d s d \phi} .
$$

Here we have used the geometric relation $\cos \alpha_{e}=\sin \psi_{e} \cos \phi$, where $\psi_{e}$ is the angle between $e^{e}$ and the normal to the plane in which the average is taken. In practice, the structure function is approximated using neighboring grid points in physical space, Kolmogorov's inertial range form being used to account for non-uniform grids (cf. $75,39)$.

$$
\begin{aligned}
\tilde{F}_{2}^{\odot}=\frac{1}{4} \sum_{k=1,2}[ & \left\|\tilde{\mathbf{U}}(\mathbf{x})-\tilde{\mathbf{U}}\left(\mathbf{x}+\Delta x_{k}^{+} \hat{\boldsymbol{\imath}}_{k}\right)\right\|^{2}\left(\frac{r}{\Delta x_{k}^{+}}\right)^{2 / 3} \\
& \left.+\left\|\tilde{\mathbf{U}}(\mathbf{x})-\tilde{\mathbf{U}}\left(\mathbf{x}-\Delta x_{k}^{-} \hat{\boldsymbol{\imath}}_{k}\right)\right\|^{2}\left(\frac{r}{\Delta x_{k}^{-}}\right)^{2 / 3}\right],
\end{aligned}
$$

where $\Delta_{k}^{+}$and $\Delta_{k}^{-}$are the grid spacing in the positive and negative $k$-direction and $r$ is the separation length for the structure function.

Finally, the subgrid energy is computed by assuming that the energy spectrum of the subgrid elements takes the form of Lundgren's stretched spiral vortex ${ }^{42}$

$$
E_{\alpha, \beta}(k)=\kappa_{0} \epsilon^{2 / 3} k^{-5 / 3} e^{-2 \nu k^{2} / 3\left|s_{\alpha, \beta}\right|} .
$$

The subgrid stress tensor is then assembled from 3.4, the total subgrid kinetic energy being $K_{\text {sgs }}=1 / 2 \tau_{i i}$. For post-priori model assessment, the dissipation is computed from

$$
\epsilon=2 \nu \hat{S}_{i j} \hat{S}_{i j}+\epsilon_{s g s}, \quad \epsilon_{s g s}=-\hat{S}_{i j} \tau_{i j}=-K \hat{S}_{i j}\left(\delta_{i j}-e_{i}^{v} e_{j}^{v}\right)
$$




\section{Chapter 4}

\section{Numerical Method}

Accurate simulations of an isolated vortex require that the numerical scheme be applied on a domain that extends to infinity in the cross plane. For this reason, a radially stretched cylindrical coordinate system is a natural choice since the boundary condition at infinity need only be applied in one direction. The downside to such a technique is that the coordinate singularity at the origin can decrease the accuracy or computational efficiency of the otherwise spectrally accurate method. Leonard and Wray ${ }^{38}$ dealt with this problem in their pipe flow code by choosing radial basis functions that allow the pole condition at the origin to be satisfied exactly by all azimuthal modes. Matsushima and Marcus ${ }^{44}$ developed a similar set of orthogonal polynomial eigenfunctions of a singular Sturm-Liouville equation to expand analytic functions on the unit disk. Later, they replaced the polynomial set by Rational Legendre Functions mapped onto the interval $0 \leq r<\infty^{45}$. Such basis functions satisfy the pole condition at the origin and remain bounded as $r \rightarrow \infty$, making them an 'appropriate choice for the computation of vortex dynamics in an unbounded domain ${ }^{45}$. Using this technique, the authors ${ }^{45}$ performed a transient simulation of the interaction of two vortex filaments. Their computation exhibits the long wavelength Crow instability as well as a faster growing short wavelength instability; however, vorticity far away from the origin had to be removed periodically to continue the computation stably. Not surprisingly, this resulted in an unphysical loss of circulation and axial momentum. Qin ${ }^{56}$ used B-splines as basis functions in the radial direction 
for their simulations of a strained vortex. These functions have the advantage that only a few modes have non-zero support at the origin; however, the resulting scheme is not spectrally accurate.

The alternative to performing computations in a cylindrical coordinate system is to employ a Cartesian grid. The benefit gained by avoiding the problems associated with a coordinate singularity is offset by the need to deal with two unbounded physical dimensions if image effects are to be eliminated. A further consequence of placing a Cartesian grid over a naturally cylindrical phenomenon is the higher number of wasted grid points outside the core region. Despite these short-comings, Sreedhar and Ragab ${ }^{66,67}$ were able to perform LES and DNS of vortices with and without axial velocity defects using a 6th order compact finite difference scheme in both cross plane directions. By stretching the grid in these directions, they imposed symmetry boundary conditions at a distance from the origin of 30 core radii. Further, they argued that the resulting image flows have a negligible effect. Cain et al. ${ }^{10}$ developed a technique for applying discrete Fourier Series to infinite domains by using a mapping designed to minimize truncation errors. They tested the technique on a vortex array, applying periodic boundary conditions at a theoretically infinite distance, hence eliminating the effect of image flows. Additional stretching in the second dimension was applied by Buell ${ }^{8}$, although the nature of his free-shear problems (i.e. the need to model inflow) was such that a finite-difference technique was used in that direction. In his compressible simulations of an isolated vortex, Qin ${ }^{56}$ applied the Cain's ${ }^{10}$ mapping in both cross plane directions. Unfortunately, severe restrictions are placed on the stretching parameters ${ }^{2}$ if the inversion of an ill-conditioned system is to be avoided during the solution of the Poisson equation inherent to the incompressible formulation.

For reasons of simplicity and computational accuracy, we pursue a numerical technique suited to the simulation of an infinite vortex array similar to that of Cain et al. 10. The strain rate field imposed by counter rotating image flows is justifiable since a single wing tip vortex shed from a lifting body is not completely isolated from the effects of its counter rotating neighbor as well as other albeit weaker vortex systems 
that form from the rear stabilizer and from flap tips. We now discuss the formulation of the numerical method and return to the repercussions associated with image vortices in Chapter 5.

\subsection{The Pseudospectral Method}

Consider the simplified geometry of a periodic box. Let $\hat{\mathbf{u}}$ and $\hat{P}$ be the Fourier coefficients of the velocity field $\mathbf{u}(\mathbf{x}, \mathbf{t})$ and pressure $P(x, t)$ defined on uniformly spaced grid points. The velocity $\mathbf{u}(\mathbf{x}, \mathbf{t})$ can therefore be expanded as

$$
\mathbf{u}(\mathbf{x}, t)=\sum_{\mathbf{k}} \hat{\mathbf{u}}(\mathbf{k}, t) e^{i \mathbf{k} \cdot \mathbf{x}}
$$

If the even number $N_{j}$ is the resolution in the $j^{\text {th }}$ direction then the wave number $k_{j}=\frac{2 \pi m}{L_{j}}$, with $m=\frac{-N_{j}}{2}, \frac{-N_{j}}{2}+1, \ldots, \frac{N_{j}}{2}-1$. Since an even number of grid points is used, the wave number corresponding to $|m|=N / 2$ is represented by only a single waveform and is typically set to 0. Applying this transformation to the Navier-Stokes equations casts them in their wave-space form

$$
\begin{gathered}
\frac{\partial \hat{u}_{i}}{\partial t}+\hat{f}_{i}^{N L}=-k_{i} \hat{P}+\nu k^{2} \hat{u}_{i}, \\
k_{i} \hat{u}_{i}=0,
\end{gathered}
$$

where $k^{2}=\mathbf{k} \cdot \mathbf{k}$, and $\hat{f}_{i}^{N L}$ is the transformed representation of the non-linear term. The spectral method proceeds by projecting the transformed Navier Stokes equations (4.1) onto the wave space $k_{i}$ and imposing the incompressibility constraint (4.2) to obtain the pressure compatibility equation (4.3).

$$
\hat{P}=\frac{k_{j} \hat{f}_{j}^{N L}}{k^{2}} .
$$


This projection makes the scheme very computationally efficient as it eliminates the pressure, allowing the governing equations to be rewritten as

$$
\frac{\partial \hat{u}_{i}}{\partial t}+\hat{f}_{i}^{N L+P}+\nu k^{2} \hat{u}_{i}=0
$$

where

$$
\hat{f}_{i}^{N L+P} \equiv\left(\delta_{i j}-\frac{k_{i} k_{j}}{k^{2}}\right) \hat{f}_{i}^{N L}
$$

Hence, an Integrating factor permits the equations to be written in the form

$$
\frac{\partial}{\partial t}\left(e^{\nu k^{2} t} \hat{u}_{i}\right)=-e^{\nu k^{2} t} \hat{f}_{i}^{N L+P}
$$

from which the evolution in time of $\hat{u}_{i}$ can be computed using a suitable time-marching scheme. Finally for reasons of computational efficiency, the non-linear term is computed in physical space and hence the method is referred to as pseudospectral.

\subsection{Dealiasing}

Computation of the non-linear term in physical space leads to aliasing errors. These result when the highest modes of the non-linear product cannot be resolved on the discrete mesh points and are folded back into the resolved modes. This can be seen by considering, in one dimension, the non-linear product $w(x)$ formed by multiplying two functions $u(x)$ and $v(x)$, which in Fourier space are represented by the discrete coefficients $u_{k}$ and $v_{k}$

$$
\check{w}_{k}=\frac{1}{N} \sum_{j=0}^{N-1} u_{j} v_{j} e^{-i k x_{j}}, \quad k=-\frac{N}{2}, \ldots, \frac{N}{2}-1 .
$$

Substituting the definitions $u_{j}=\sum_{m=-N / 2}^{N / 2-1} \check{u}_{m} e^{i m x_{j}}$ and $v_{j}=\sum_{n=-N / 2}^{N / 2-1} \check{u}_{n} e^{i n x_{j}}$ for $j=0,1, \ldots, N-1$ into the expression for the product and using the orthogonality 
relation

$$
\frac{1}{N} \sum_{j=0}^{N-1} e^{i(n+m-k) x_{j}}= \begin{cases}1 & \text { if }(n+m-k)=N p, p=0, \pm 1, \pm 2, \ldots \\ 0 & \text { otherwise }\end{cases}
$$

the product can be written as

$$
\check{w}_{k}=\sum_{m+n=k} \check{u}_{m} \check{v}_{n}+\sum_{m+n=k \pm N} \check{u}_{m} \check{v}_{n}
$$

where the first term is the discrete representation of the product and the second term the aliasing error. Clearly the error is dominated by the highest wave mode contribution. Hence, while such errors should be small for a DNS, the highest wave modes in an LES contain significant amounts of energy (the smallest scales are well above the dissipative range) and could have adverse effects on the simulation. To overcome this problem, current simulations use the $2 / 3$ rule dealising technique. In this scheme, only $2 / 3$ of the $N$ modes are used for resolving the flow variables, the remaining modes being set to 0 . To see how this affects the aliasing error term, consider the worst case of the highest resolved mode, $k=-2 / 3 N / 2=-N / 3$. The second term in 4.8 could only contribute for $m+n=-N / 3+N=2 N / 3$ which

can only be true if at least one of the factors is a mode outside the resolved range $m, n \geq N / 3$. Hence the aliasing error only affects modes outside the resolved scales (which are re-set to zero after the transform). In three-dimensions, the $2 / 3$ rule is imposed in each direction, and the coefficients are further subjected to spherical truncation (cf. Ref. ${ }^{11}$ ).

\subsection{Cain's Mapping}

Motivated by the need for accurate differentiation and integration schemes for domains where the boundary conditions are imposed far from the region of physical interest, Cain et al. ${ }^{10}$ devised a mapping which yields alias free operators with trun- 
cation error that is generally small. A brief discussion of this scheme is now presented. Consider any function $f(x, y, z, t)$ in physical space which is transformed in the $\mathrm{z}$ direction to the computational coordinate $\eta$ through the mapping $z=h(\eta)$. The derivatives in the two coordinate systems being related by the chain rule:

$$
\frac{\partial f}{\partial z}=\frac{\partial f}{\partial \eta} \frac{d \eta}{d z}=\frac{1}{h^{\prime}} \frac{d f}{d \eta}
$$

The function in computational space can be expressed in terms of a discrete Fourier series involving the $\left(N_{x}-1\right) \times\left(N_{y}-1\right) \times\left(N_{\eta}-1\right)$ equi-spaced computational nodes as well. Any derivative in physical space can then be obtained by multiplying these terms in the representation of the function in computational space by the terms in the Fourier series representation of the metric $1 / h^{\prime}(\eta)$. In general $N_{\eta}$ terms are required and the resulting physical space derivative contains $N+N_{\eta}-1$ waveforms describing the behavior in the $z$ direction. Truncating the results to $N_{\eta}$ terms produces potentially large errors. Cain et al. were able to greatly reduce these truncation errors by restricting the mapping functions to those which contain only a few Fourier modes with small wave numbers. Of particular interest to the current work is the mapping $z=h(\eta)=-b \cot (\eta), \quad 0 \leq z<\infty$ and the associated metric

$$
\frac{1}{h^{\prime}(\eta)}=\frac{1}{b}\left(1-\frac{e^{i \eta}+e^{-i \eta}}{2}\right)
$$

Using this mapping, the physical space derivative is written

$$
\frac{\partial f(x, y, z, t)}{\partial z}=\sum\left(i k_{n_{\eta}} \hat{f}_{n_{\eta}}-\frac{i k_{n_{\eta}-1}}{2} \hat{f}_{n_{\eta}-1}-\frac{i k_{n_{\eta}+1}}{2} \hat{f}_{n_{\eta}+1}\right) e^{i k_{n_{x}} x_{i}+i k_{n_{y}} y_{j}+i k_{n_{\eta}} z_{k}}
$$

where

$$
k_{n_{\eta}}= \begin{cases}\frac{2 \pi n_{\eta}}{N_{\eta}} & \text { if } 0 \leq n_{\eta} \leq N_{\eta} \\ 0 & \text { otherwise }\end{cases}
$$




\subsection{A Spectral Collocation Method}

Spectral collocation methods ${ }^{11}$ are based on the physical space form of the NavierStokes equations evaluated at discrete collocation points. Upon integration over one time step $\delta t$ one obtains, in semi-discrete form

$$
u_{i}^{n+1}-u_{i}^{n}=-\int_{t_{n}}^{t_{n+1}} \frac{\partial P}{\partial x_{i}} d t+\nu \int_{t_{n}}^{t_{n+1}} L\left(u_{i}\right) d t+\int_{t_{n}}^{t_{n+1}} N L\left(u_{i}\right) d t
$$

where, $L\left(u_{i}\right)$ represents the linear term, and $N L\left(u_{i}\right)$ the non-linear term written in skew-symmetric form to reduce aliasing effects. For efficiency reasons, the non-linear term is evaluated using an explicit scheme of order $J_{e}$ while for stability reasons, the non-linear term via an implicit scheme of order $J_{i}$. If the pressure is treated in a fully implicit manner, solution of the semi discrete system can be split into 3 substeps

$$
\begin{gathered}
\frac{\hat{u}_{i}-u_{i}^{n}}{\delta t}=\sum_{q=0}^{J_{e}-1} \beta_{q} N L\left(u_{i}^{n-q}\right), \\
\frac{\hat{\hat{u}}_{i}-\hat{u}_{i}}{\delta t}=-\frac{\partial P^{n+1}}{\partial x_{i}}, \\
\frac{u_{i}^{n+1}-\hat{\hat{u}}_{i}}{\delta t}=\nu \sum_{q=0}^{j_{i}-1} \gamma_{q} L\left(u_{i}^{n+1-q}\right) .
\end{gathered}
$$

By taking the divergence of the pressure equation 4.11 and enforcing continuity on the updated velocity field, a Poisson equation for the pressure is obtained

$$
\frac{\partial^{2} P^{n+1}}{\partial x_{i}^{2}}=\frac{1}{\delta t} \frac{\partial \hat{u}_{i}}{\partial x_{i}}
$$

When this equation is solved in wave space, the strictly diagonal structure results in a decoupled system allowing explicit calculation of the pressure. This step is analogous to the removal of the pressure altogether in the pseudospectral method by the projection of the Navier-Stokes equations onto the wave space $k_{i}$. 


\subsubsection{Time Integration}

Rather than using standard Adams-type explicit schemes for time integration, a stiffly stable scheme is used ${ }^{30}$. This scheme is based on backwards differentiation of the time derivative and results in a larger stability region. Incorporating this scheme, the overall splitting method takes the form ${ }^{30}$

$$
\begin{aligned}
\frac{\gamma_{0} \hat{u}_{i}-\sum_{k=0}^{J-1} \alpha_{k} u_{i}^{n-k}}{\delta t} & =\sum_{q=0}^{J_{e}-1} \beta_{q} N L\left(u_{i}^{n-q}\right), \\
\frac{\hat{\hat{u}}_{i}-\hat{u}_{i}}{\delta t} & =-\frac{\partial P^{n+1}}{\partial x_{i}}, \\
\frac{\gamma_{0} u^{n+1}-\hat{u}_{i}}{\delta t} & =\nu L\left(u_{i}^{n+1}\right) .
\end{aligned}
$$

The coefficients $\alpha, \beta$ and $\gamma$ are presented in Appendix B.

\subsubsection{Poisson/Helmholtz Equations}

Stretching the infinite physical domain onto a uniformly spaced finite computational grid introduces a coupling between wave modes, which generally prevents the explicit inversion of the Poisson equation essential to the efficiency of the pseudospectral method. The Cain's mapping presented in Section 4.3 limits this coupling to just one wave mode above and below for first derivatives and two wave modes above and below for second derivatives. The two periodic directions decouple and hence, inversion of the pressure-Poisson equation (4.11) in the computational wave space reduces to the solution of a Helmholtz equation. The final step in the split method involves advancing the viscous terms, and due to the necessity of an implicit treatment, results also in the solution of a Helmholtz equation. Due to the minimal coupling in the stretched coordinate direction, inversion of the pressure-Poisson equation (4.11) and the viscous Helmholtz equation in the computational wave space amounts to solution of a penta-diagonal system and hence much of the speed benefits associated with the pseudospectral method are retained. 
Consideration of the boundary conditions that must be imposed on the system is a non trivial matter. Cain et al. ${ }^{10}$ imposed zero pressure and velocity at infinity in their simulations by setting the 0 frequency mode in the stretched direction $\left(k_{\eta}=0\right)$ to zero magnitude. This only works for functions that exhibit 'odd' behavior in this direction as they average to zero. In addition, it results in small but never the less non-zero derivatives at infinity and a resulting violation of the divergence free constraint. Buell ${ }^{8}$ placed constraints on the system that force the two highest modes to have zero magnitude as well as satisfying the condition at infinity. We choose to cautiously ignore the truncation error associated with the mapping since for a well resolved simulation the resulting error should be small; for large eddy simulations this may not be the case and is investigated later. The correct boundary condition can be derived by considering the Fourier representation of a general flow variable $\phi$.

$$
\phi(x, y, \eta)=\sum_{k_{x}} \sum_{k_{y}} \sum_{k_{\eta}} \hat{\phi}_{k_{x} k_{y} k_{\eta}} e^{i k_{x} x} e^{i k_{y} y} e^{i k_{\eta} \eta}
$$

The point $\eta=0$ maps to $z=\infty$ and hence the correct boundary condition is derived as a sum over all non-zero stretched modes.

$$
\hat{\phi}_{k_{x} k_{y} 0}=-\sum_{k_{\eta} \neq 0} \hat{\phi}_{k_{x} k_{y} k_{\eta}}
$$

\subsubsection{Parallel Implementation}

Coupling between wave-numbers is introduced predominantly during the non-linear step since the use of Fourier basis functions in two directions decouples the system into a series of Helmholtz equations during the pressure and viscous steps. The stretching function introduces some coupling in the unbounded direction, however, by parallelizing in one of the strictly periodic physical directions, the majority of communication can be restricted to the calculation of the non-linear term and more specifically the associated Fourier Transform. The FFTW package ${ }^{18}$ is used for computing these transforms since it is computationally efficient and parallel. While it only allows par- 
allelization in one dimension, the increase in speed possible from allocating processors across the second direction is small for the modestly sized computational grids used in the current work.

Since the physical space representation of all flow variables is strictly real valued, the complex multi-dimensional Fourier series enjoys a Hermitian symmetry.

$$
\hat{\phi}(i, j, k)=\hat{\phi}\left(N_{i}-i, N_{j}-j, N_{\eta}-\eta\right)^{*}
$$

Storage of complex arrays takes advantage of this by only storing $N_{\eta} / 2+1$ complex values. Strictly speaking, $N_{\eta} / 2$ values are only needed; however, array structure is simplified in the FFTW package by storing the additional modes which are in fact redundant due to the symmetry

$$
\hat{\phi}\left(i, j, N_{\eta / 2+1}\right)=\hat{\phi}\left(N_{i}-i, N_{j}-j, N_{\eta} / 2-1\right)^{*}
$$

This symmetry and hence some inter-processor communication is needed when imposing the boundary condition at infinity.

\subsection{Test Cases}

The simulation code described previously was written specifically for the present work and it is thus necessary to test it rigorously. This phase is typically broken down into two phases, namely verification and validation. The first of these ensures that the code converges to the solution of the original PDE, while the latter involves checking that the governing equations are indeed a good approximation of reality. Verification is typically done by comparison with Direct Numerical Simulation or known analytical solutions. Validation with experimental results for the present code is difficult due to its highly specialized nature arising from the importance of boundary conditions in the incompressible framework. We begin then by verifying correct implementation and consider code validation later, when we examine the simulation of the interaction of trailing vortices. 


\subsubsection{Strained Vortex}

The presence of periodicity, while simplifying the numerics, introduces the issue of image flows. For example, in a two-dimensional domain described in cylindrical coordinates and unbounded in the radial direction, a distribution of vorticity with $\omega \propto e^{-r^{2}}$ will simply diffuse away in a self-similar manner. If, as in the present computational domain, periodicity is imposed in 1 of these unbounded directions, the resulting images have the effect of subjecting the vortex to a straining effect. The equilibrium configuration that results from the imposition of a non-axisymmetric strain rate field has been studied ${ }^{59,9}$. While these studies were able to explicitly impose strain rate fields of various strengths, ours is the implicit result of the boundary conditions; however, qualitative comparison is possible. A more detailed description of the resulting strain rate magnitude is presented in Appendix A; for now we just note that the eigenvectors are at 45 degrees to the direction of periodicity. Figure 4.1 shows

contours of vorticity for an (initial distribution $\propto e^{-r^{2}}$ ) after one transit time defined as $t_{T}=2 \pi r_{0} / v_{0}, r_{0}$ and $v_{0}$ being the location of and magnitude of maximum initial tangential velocity at two Reynolds number $R e=\frac{\Gamma}{2 \pi}=30,100$. Re can be viewed as a measure of the strength of inertial effects relative to those induced by the implicit strain rate field and viscous diffusion. At the lower Re, the vortex is stretched appreciably in the direction of the maximum strain rate, a balance between rotation and this strain rate determining the final angle of the major axis. As Re is increased, Buntine and Pullin ${ }^{9}$ (BP henceforth) found that the vortex becomes stronger, and the directional effects of the strain rate are overcome to the extent where the resulting equilibrium resembles the results for a Burgers vortex in an axisymmetric strain rate field.

By decreasing the ratio of the vortex core size to the length of the periodic domain, the influence of the image flow strain rate can be reduced to the extent where the diffusing vortex can be approximated by the self-similarly decaying line vortex

$$
\omega(r, t)=\frac{\Gamma}{4 \pi \nu t} e^{-r^{2} / 4 \nu t}
$$



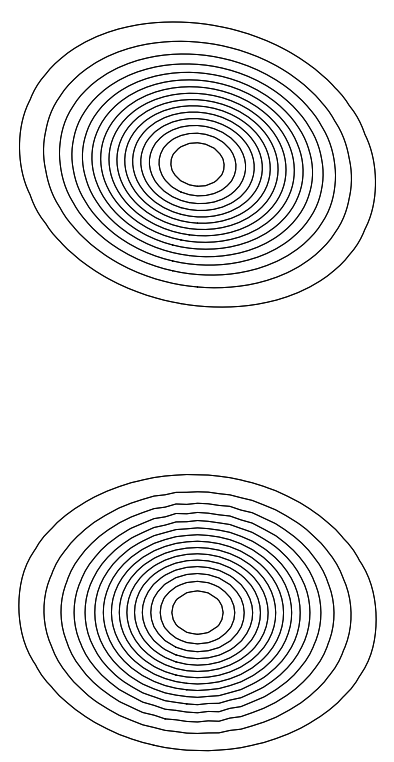

Figure 4.1: Contours of vorticity for an initially Gaussian distribution, one transit time later. Top picture is $R e=30$, bottom is $R e=100$.

Figure (4.2) shows a comparison of vorticity profiles through the core of the vortex at two time instances for the $R e=1000$ simulation and the corresponding axisymmetric solution. Although the peak velocities match very well however, there is a slight discrepancy in the tails which can be attributed to the presence of image flows. This provides a good indication that the viscous terms are being implemented properly. To check the pressure solver, the comparison is explored further by considering in Figure (4.3) the pressure drop in the core of the vortex.

\subsubsection{Co-rotating vortex merger}

We now move on to the more complicated problem of co-rotating vortex merger in a bid to examine the implementation of the non-linear terms. The benchmark for this test case is the computational work of BP who studied the influence of an imposed strain rate field on vortex phenomena. Their strain rate field was of the form

$$
\mathbf{u}_{s}=\beta(t) x_{1} \hat{\mathbf{i}}+[\beta(t)-\gamma(t)] x_{2} \hat{\mathbf{j}}+\gamma(t) x_{3} \hat{\mathbf{k}}
$$




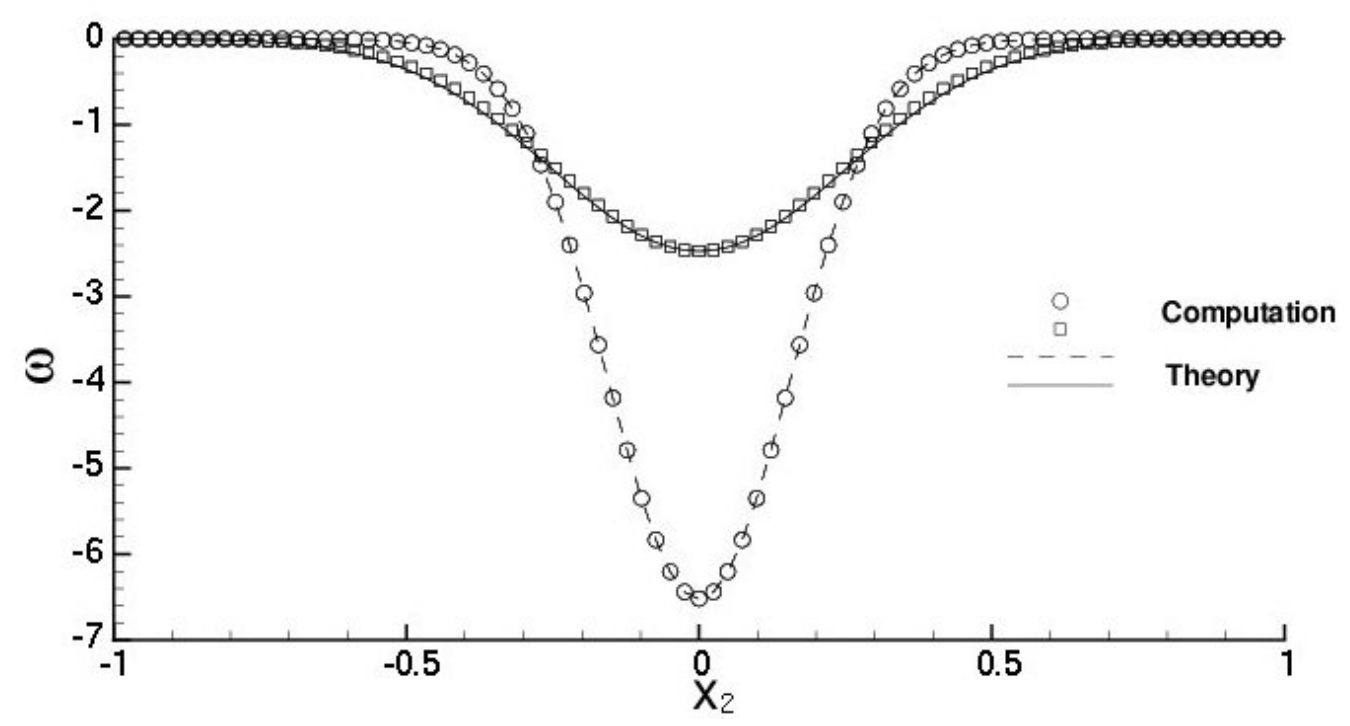

Figure 4.2: Vorticity evolution. Comparison between computation and the theoretical diffusing vortex, $R e=1000$.

where $\beta(t)$ and $\gamma(t)$ are strain rates. When $\beta=1 / 2 \gamma$, the strain rate field is axisymmetric, and, providing the velocity vanishes at infinity, the resulting vorticity distribution, $\omega_{s}\left(x_{1}, x_{2}, t\right)$, can be related to that in the absence of a strain rate field, $\omega(\xi, \eta, \tau)$, by

$$
\omega(\xi, \eta, \tau)=\omega_{s}\left(x_{1}, x_{2}, t\right) e^{-A(t)}
$$

where

$$
\xi=x_{1} e^{1 / 2 A(t)}, \quad \eta=x_{2} e^{1 / 2 A(t)}, \quad \tau=\int_{0}^{t} e^{A\left(t^{\prime}\right)} d t^{\prime}
$$

and

$$
A(t)=\int_{0}^{t} \gamma\left(t^{\prime}\right) d t^{\prime}
$$

The initial condition for the simulation comprises two axisymmetric vorticity distributions of equal circulation $\Gamma / 2$ offset from the origin by a distance $x_{2 i}$.

$$
\omega\left(x_{1}, x_{2}, 0\right)=\frac{\Gamma}{2 \pi}\left(e^{-\left(x_{2}-x_{2 i}\right)^{2}-x_{3}^{2}}+e^{-\left(x_{2}+x_{2 i}\right)^{2}-x_{3}^{2}}\right) .
$$

For comparative purposes $x_{2 i}=2$ was chosen; however, the later stages of merger 


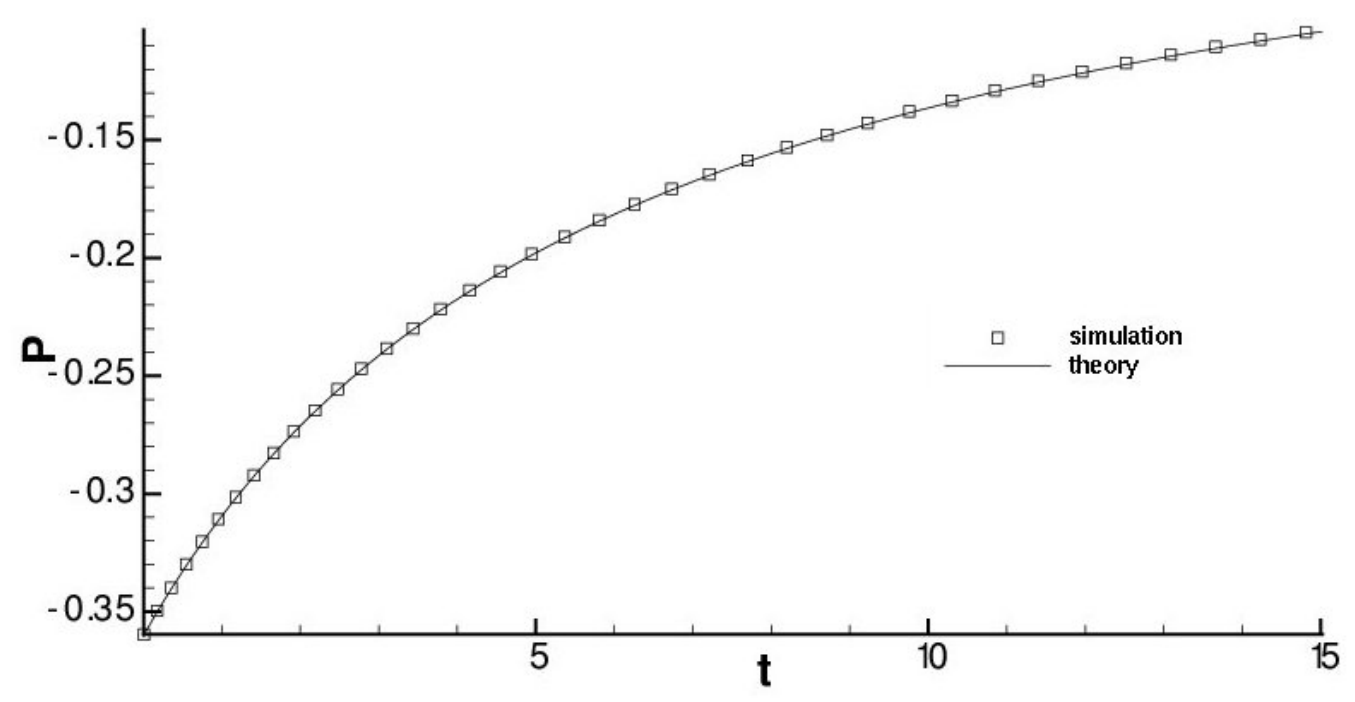

Figure 4.3: Pressure evolution over time for the diffusing vortex. $R e=1000$.

are not strongly dependent on $x_{2 i}$, provided $x_{2 i}>1^{9}$. Simulations were performed at $R e=\Gamma / 2 \pi=160$ and 640 , the transformation defined in 4.17 being used with $\beta=2$, to compute $\omega_{s}$ for comparison with BP. Results are compared qualitatively, noting once again that a non-axisymmetric strain rate field does exist in the present simulations due to the presence of image vortices (the period of the y domain is presently $16 \pi$ to minimize this effect). A comparison is shown with the previous work in Figures 4.4-4.5 and 4.6-4.7 for $R e=160$ and 640 respectively . The early stage of vortex merger is essentially that of two point vortices whereby the two simply rotate around each other. During this process however the cores spiral towards each other, the rotation rate increasing to preserve angular momentum. As the cores merge together into a large vorticity region, the leading edges are stretched considerably in the azimuthal direction, creating large gradients of vorticity. These high gradients are diffused out by viscosity on a very short timescale. At later, times the merger process becomes complete, leaving a single approximately axisymmetric vortex. The effect of $R e$ is clearly seen in that the strong gradient regions as well as the vortex arms lengthen into thinner spiral bands before the relatively weaker viscosity diffuses them. Very good agreement between the two studies is found, slight discrepancies, 
particularly within the cores at later time, being attributed to the additional straining effect of the image flows in the present simulation.

\subsubsection{Axial Velocity in 2D-3C Flows}

Continuing with the merger of like signed vortices, we now consider the evolution of the axial velocity. If no axial gradients exist (i.e. 2D-3C), the axial momentum equation behaves analogous to the transport of a passive scalar, and identical to that governing the evolution of the vorticity,

$$
\frac{\partial u_{1}}{\partial t}+u_{j} \frac{\partial u_{1}}{\partial x_{j}}=\frac{1}{R e} \frac{\partial^{2} u_{1}}{\partial x_{j}^{2}}
$$

Hence, given the same initial conditions, the axial velocity will evolve identical to the vorticity. While this gives further indication that the code is operating as expected, results are presented for the more physically interesting evolution of initial axial velocities differing from the underlying vorticity. We first consider the case in which the profiles are identical except the superposed axial velocity on one vortex is of opposite sign

$$
u_{1}\left(x_{1}, x_{2}, 0\right) \propto e^{-\left(x_{2}-x_{2 i}\right)^{2}-x_{3}^{2}}-e^{-\left(x_{2}+x_{2 i}\right)^{2}-x_{3}^{2}} .
$$

Figure (4.8) shows that initially, the axial velocity winds up similarly to the vorticity, however, due to the opposing sense of the axial velocity, cancellation occurs and a constant state will ultimately prevail. Next, we repeat the simulation for initial distributions of axial velocity once again proportional to the vorticity but rotated 90 degrees about the origin,

$$
u_{1}\left(x_{1}, x_{2}, 0\right) \propto e^{-x_{2}^{2}-\left(x_{3}-x_{3 i}\right)^{2}} \pm e^{-x_{2}^{2}-\left(x_{3}-x_{3 i}\right)^{2}} .
$$

Figure (4.9) shows a comparison between the case where the velocity distributions have equal sense and that where they are of opposite sense. During both simulations, the two distributions appear to merge, however, whereas the former develops a core region similar to the vorticity, the opposing distributions in the latter cause cancel- 

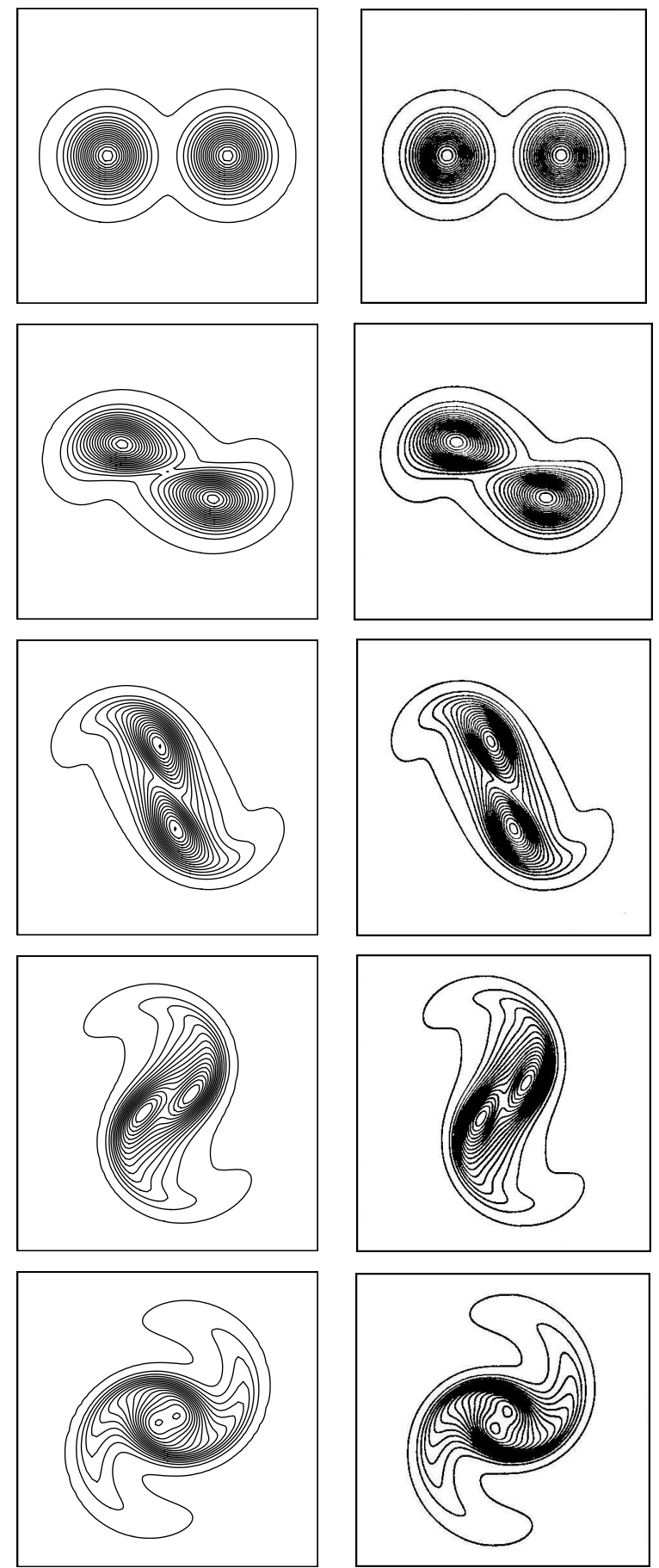

Figure 4.4: Merger of two like signed vortices in an axisymmetric strain rate field. Comparison between the present work(left) and Buntine and Pullin ${ }^{9}$ (right) at Re $=160$. The minimum contour is at $\left|\omega_{s}\right|=1.0$ and the intervals are $\left|\Delta \omega_{s}\right|=9.0$. Times are (from top to bottom) $\mathrm{t}=0,0.05,0.1,0.15,0.2$. 

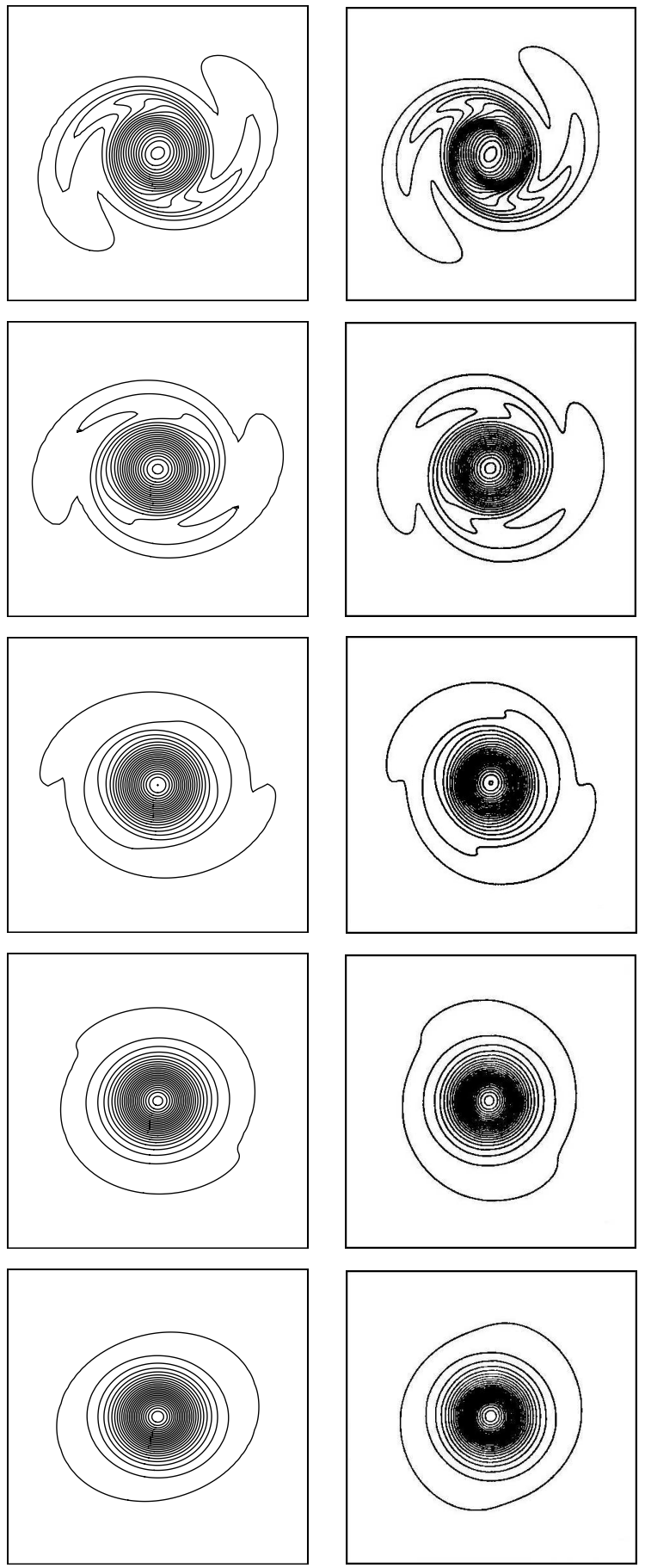

Figure 4.5: Merger of two like signed vortices in an axisymmetric strain rate field. Comparison between the present work(left) and Buntine and Pullin ${ }^{9}$ (right) at Re $=160$. The minimum contour is at $\left|\omega_{s}\right|=1.0$ and the intervals are $\left|\Delta \omega_{s}\right|=9.0$. Times are (from top to bottom) $\mathrm{t}=0.25,0.3,0.35,0.4,0.45$. 

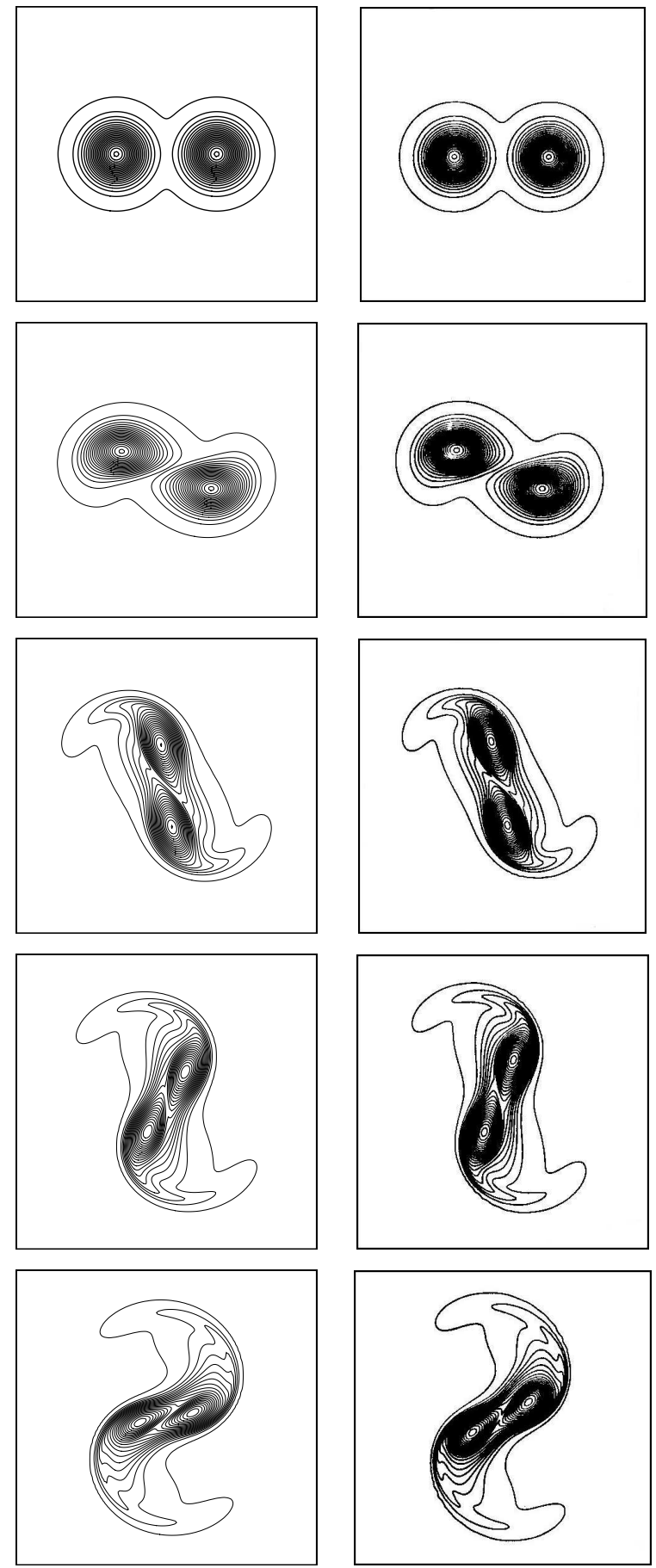

Figure 4.6: Merger of two like signed vortices in an axisymmetric strain rate field. Comparison between the present work(left) and Buntine and Pullin ${ }^{9}$ (right) at $R e=640$. The minimum contour is at $\left|\omega_{s}\right|=3.0$ and the intervals are $\left|\Delta \omega_{s}\right|=30.0$. Times are (from top to bottom) $\mathrm{t}=0,0.01,0.03,0.04,0.05$. 

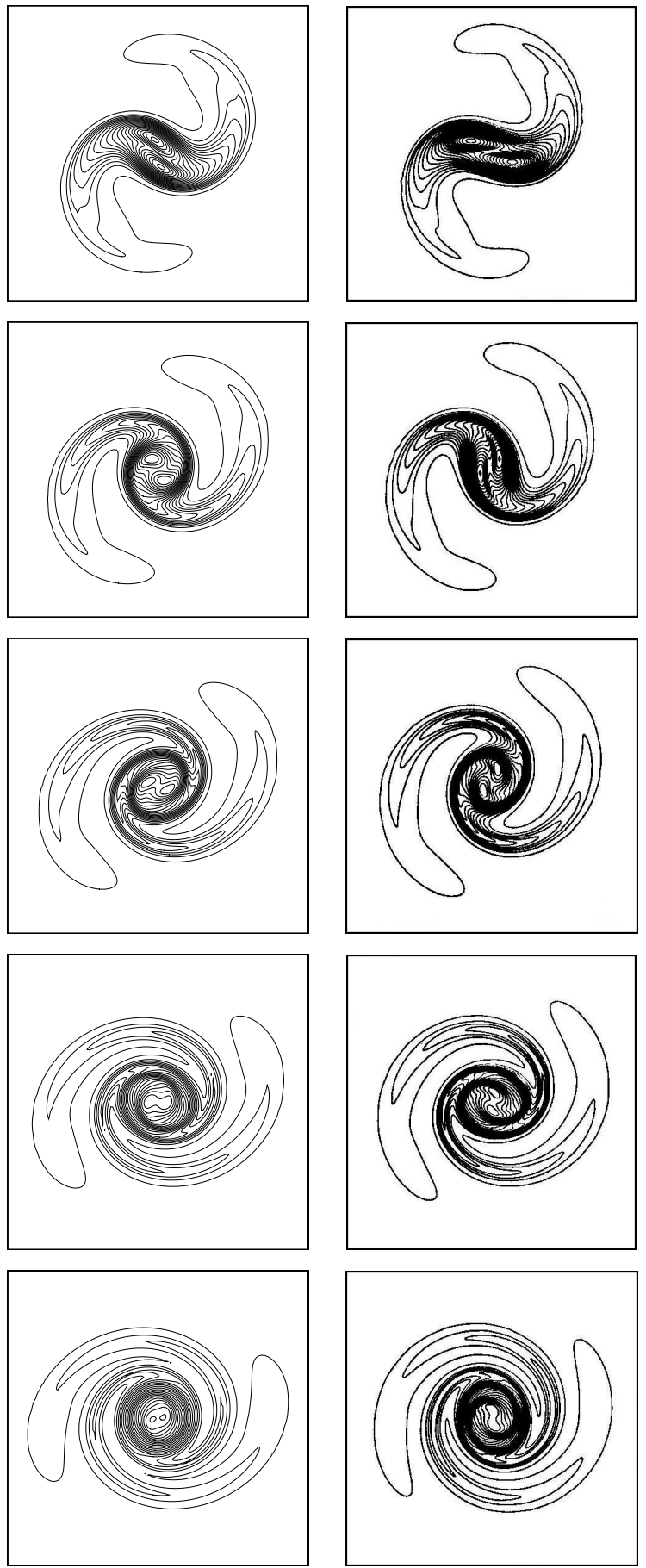

Figure 4.7: Merger of two like signed vortices in an axisymmetric strain rate field. Comparison between the present work(left) and Buntine and Pullin ${ }^{9}$ (right) at $R e=640$. The minimum contour is at $\left|\omega_{s}\right|=3.0$ and the intervals are $\left|\Delta \omega_{s}\right|=30.0$. Times are (from top to bottom) t=0.06, 0.07, 0.08, 0.09, 0.10. 
lation in the core. While no comparison can be made with proven data for these test cases, the results make physical sense.

The test cases presented so far highlight the difficulty associated with imposing boundary conditions in incompressible simulations. In the next section, we develop an initial condition for trailing vortices that is an exact solution to the Euler equations on our computational domain. Using this, we will be able to conduct a more quantitative code validation.
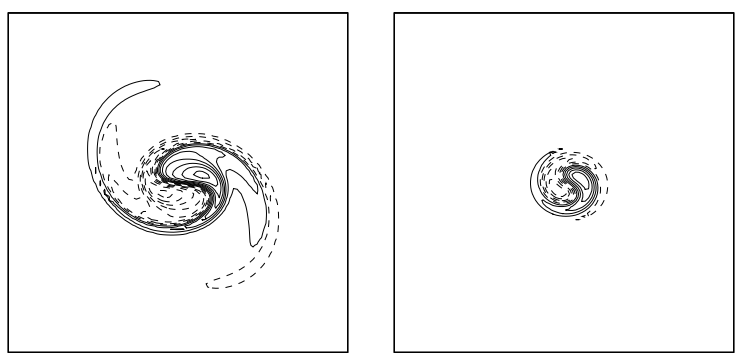

Figure 4.8: Passive scalar transport during the merger of two like signed vortices at $R e=640$. The initial distribution of the passive scalar is identical to that of vorticity in magnitude however they are of opposite sign.
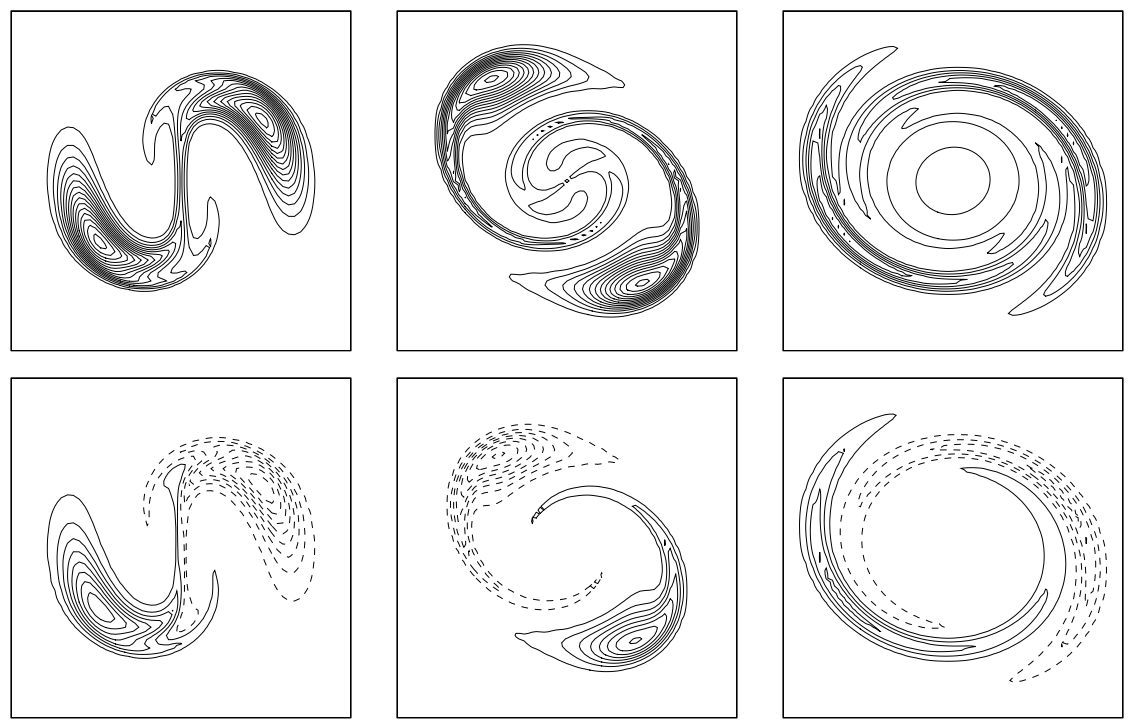

Figure 4.9: Passive scalar transport during the merger of two like signed vortices at $R e=640$. The initial distribution of the passive scalar is identical to but 90 degrees out of phase with the vorticity. Same sign (top) and opposite sign (bottom). 


\section{Chapter 5}

\section{Simulating Trailing Vortices}

Presently, we consider a model for trailing vortices based on an initial condition comprising an array of counter rotating vortices that is an exact solution to the incompressible Euler Equations in 2D. Whereas the BV represents an isolated vortex, the array provides a means of modeling the straining influence of the counter-rotating neighbor present in a true aircraft wake. In Section 5.1, we define the flow geometry and formulate the governing equations for analyzing this flow. An axial velocity that is modeled on vortex roll-up and chosen proportional to the initial axial vorticity is superimposed with a corresponding swirl parameter $q$ chosen analogous to the BV. In Chapter 6, we study the linear stability of the resulting flow for various q. These results are used to interpret the non-linear interactions that result from DNS and LES in Chapters 7 and 8.

\subsection{Flow Geometry and Formulation}

In dimensionless co-ordinates to be defined subsequently, the incompressible NavierStokes equations (N-S) are

$$
\begin{aligned}
\frac{\partial u_{j}}{\partial x_{j}} & =0 \\
\frac{\partial u_{i}}{\partial t}+u_{j} \frac{\partial u_{i}}{\partial x_{j}} & =-\frac{\partial P}{\partial x_{i}}+\frac{1}{R e} \frac{\partial^{2} u_{i}}{\partial x_{j}^{2}}
\end{aligned}
$$


and the convection-diffusion equation for a passive scalar $c$ is

$$
\frac{\partial c}{\partial t}+u_{j} \frac{\partial c}{\partial x_{j}}=\frac{1}{R e S c} \frac{\partial^{2} c}{\partial x_{j}^{2}}
$$

where $R e$ and $S c=\nu / D$ are the Reynolds and Schmidt numbers respectively, D being the scalar diffusivity. Equations (5.1) and (5.2) are applied in Cartesian coordinates on a physical domain that is periodic in the $x_{1}$ and $x_{2}$ directions and extends to infinity in $x_{3}$. In what follows, we will consider an array of vortices nominally aligned with the $x_{1}$ direction whose centers are initially on the $x_{2}$ axis.

\subsubsection{Initial Condition; $x_{2}^{*}-x_{3}^{*}$ plane}

In the present formulation, the vortex axis is chosen along one of the strictly periodic directions ( $x_{1}$ direction); however, the presence of a second periodic direction $\left(x_{2}\right)$ in the plane of the vortex results in an array of image flows. An initial condition can be derived from the Mallier\& Maslowe ${ }^{43}(\mathrm{M} \& \mathrm{M})$ vortex, an exact solution of the inviscid Euler equations which is the counter-rotating analogue of the Stuart vortex array ${ }^{70}$. Making quantities non-dimensional by choice of a length scale $L^{*} / 2 \pi$, where $L^{*}$ is the $x_{2}^{*}$-period of the array and velocity scale $\Gamma^{*} / L^{*}$, where $\Gamma^{*}$ is the circulation of each

member of the array, then the streamfunction for the M\&M vortex can be expressed as

$$
\psi\left(x_{2}, x_{3}\right)=\frac{1}{2} \log \left(\frac{\cosh \left(C x_{3}\right)-C \cos \left(x_{2}\right)}{\cosh \left(C x_{3}\right)+C \cos \left(x_{2}\right)}\right),
$$

where $C \in[-1 ; 1]$ is the concentration parameter. When $C=0, \psi=0$, while for $C \rightarrow \pm 1$ the streamlines become more circular until the counter-rotating point-vortex array is recovered at $C= \pm 1$. The Reynolds number in (5.1-5.2) is then $R e=\Gamma^{*} / 2 \pi \nu^{*}$ and the dimensionless vortex circulation is $\Gamma=2 \pi$. The dimensionless vorticity $\omega_{i}$ is

$$
\omega_{1}\left(x_{2}, x_{3}\right)=-\Delta^{2} \psi=\frac{\left(1-C^{2}\right)}{4} \sinh (4 \psi), \quad \omega_{2}=\omega_{3}=0
$$


while the velocity components are

$$
\begin{aligned}
& u_{2}\left(x_{2}, x_{3}\right)=\frac{C^{2} \sinh \left(C x_{3}\right) \cos \left(x_{2}\right)}{\cosh ^{2}\left(C x_{3}\right)-C^{2} \cos ^{2}\left(x_{2}\right)}, \\
& u_{3}\left(x_{2}, x_{3}\right)=-\frac{C \cosh \left(C x_{3}\right) \sin \left(x_{2}\right)}{\cosh ^{2}\left(C x_{3}\right)-C^{2} \cos ^{2}\left(x_{2}\right)} .
\end{aligned}
$$

Figure 5.1(a) shows the vorticity distribution for the M\&M vortex array with $\mathrm{C}=0.9$. In order to characterize the vortex separation in this array, one must first define a length scale indicative of the vortex size. Studies of the LOV typically choose the radius at which the azimuthal velocity takes its maximum value $\left(r_{c} / \delta \approx 1.12091\right)$ 66,67 , the distance at which boundaries or other vortices are present being described in terms of this core size. For the $\mathrm{M} \& \mathrm{M}$ vortex however, the streamlines are only cylindrical in the limit $C \rightarrow \pm 1$; the straining influence of the array introduces ellipticity. We consider the plane defined by $x_{3}=0$ and define the core size as the distance $r_{c}$ from the core of each vortex $x_{2_{c}}=n \pi$ ( $n$ integer) to the location $x_{2_{\max }}$ at which $u_{3}$ takes its maximum value. Thus

$$
\begin{aligned}
& u_{3_{\max }} \equiv u_{3}\left(x_{2_{\max }}\right)= \pm \frac{1}{2 \sqrt{1-C^{2}}} \\
& r_{c}=\left|x_{2_{\max }}-x_{2_{c}}\right|= \pm \frac{1}{2} \cos ^{-1}\left(\frac{3 C^{2}-2}{C^{2}}\right) .
\end{aligned}
$$

For $C=0.9$ (Figure 5.1(b)) $r_{c} \approx 0.506$. Increasing $C$ decreases $r_{c}$ and hence increases the separation size, while providing an initial condition that always satisfies the unsteady Euler equations. For the present work, all simulations are performed with $C=0.9$ which corresponds to a separation distance $\approx 6.2 r_{c}$.

\subsubsection{Axial Velocity}

As mentioned earlier, the Lamb-Oseen vortex is stable to linear perturbations unless

an axial velocity of the form $u^{*}=u_{d}^{*} e^{-\left(r^{*} / \delta^{*}\right)^{2}}$ is added. The resulting vortex, termed 
(a)

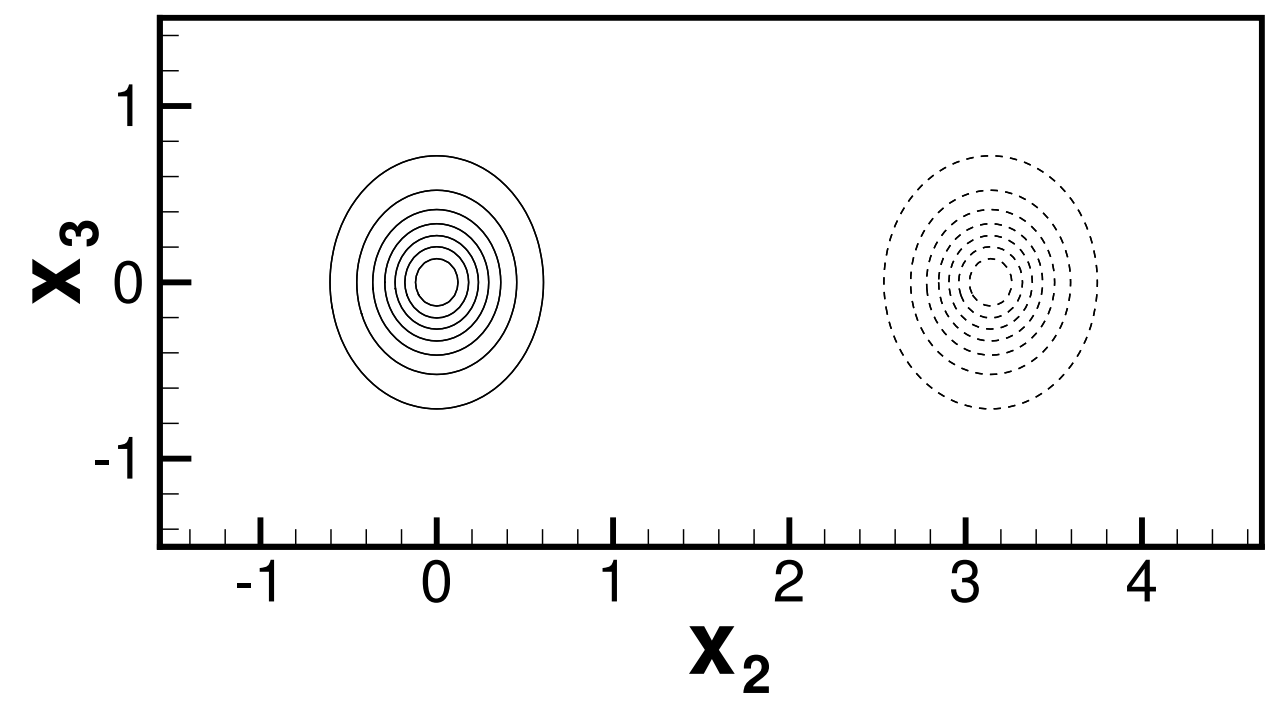

(b)

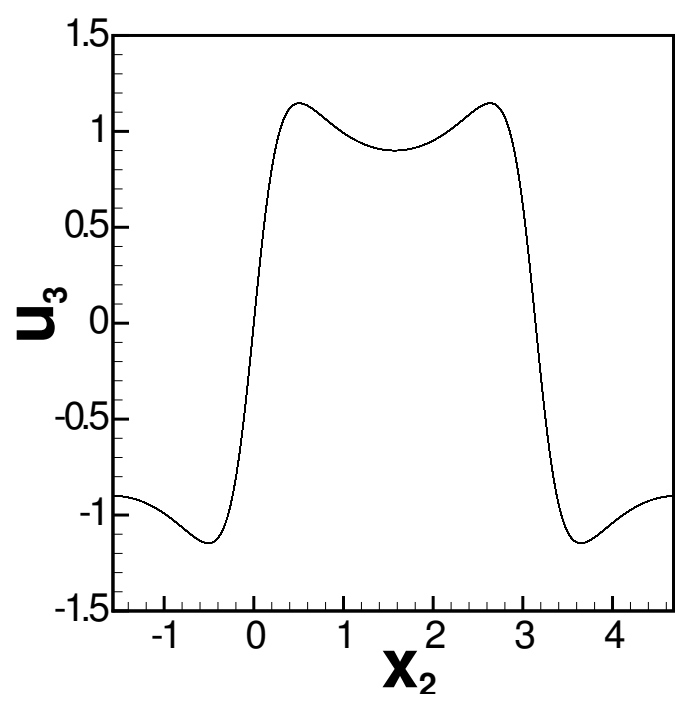

Figure 5.1: (a) (left) Contours of $\omega_{1}$ and (b) (right) distribution of $u_{3}$ along $x_{3}=0$ for one period of the $\mathrm{M} \& \mathrm{M}$ vortex array with $\mathrm{C}=0.9$. 

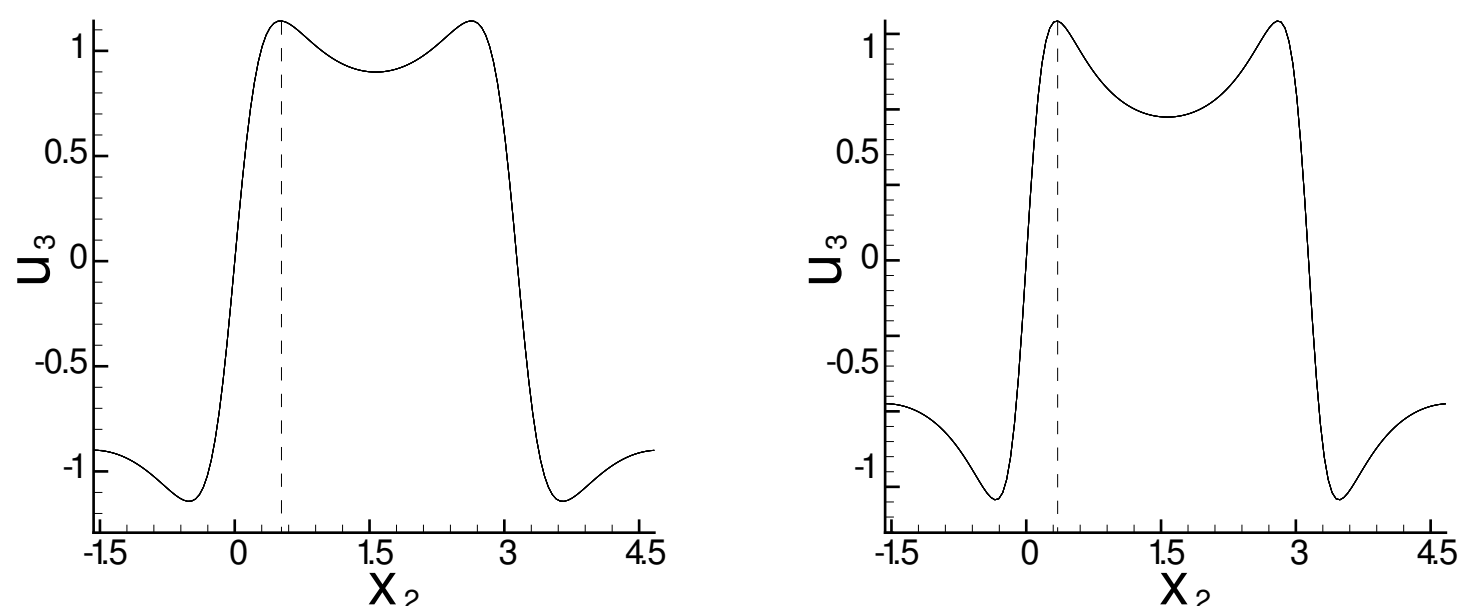

Figure 5.2: Velocity profile through center of vortex array showing definition of the core radius. Left $\mathrm{c}=0.9$ gives $r_{c}=0.506$ and right $\mathrm{c}=0.95$ has $r_{c}=0.33491$.

the Batchelor or q vortex is characterized by the swirl parameter q defined as

$$
q=\frac{\Gamma}{2 \pi \delta^{*} U_{d}^{*}}
$$

Once again, the resulting vortex is only an exact solution to the Euler equations on a radially infinite domain. Using $(1.1),(1.2)$ can be rewritten in terms of the ratio of the swirling velocity $u_{\theta_{\max }}^{*}$ to the maximum axial velocity $u_{d}^{*}$

$$
q \approx 1.56 \frac{u_{\theta_{\max }^{*}}^{*}}{u_{d}^{*}}
$$

We retain this definition of $q$, replacing $u_{\theta_{\max }}^{*}$ with $u_{3_{\max }}$ and $u_{d}^{*}$ with its non-dimensional equivalent. An exact solution to the 2D-3C Euler equations can be constructed with any axial velocity distribution that is a function only of $\psi$. Presently we choose

$$
u_{1}\left(x_{2}, x_{3}\right)=\frac{1.56 u_{3_{\max }}}{q_{0}}\left|\frac{\omega_{1}\left(x_{2}, x_{3}\right)}{\omega_{1}(0,0)}\right| .
$$

Where the subscript in $q_{0}$ distinguishes the chosen initial swirl parameter from its transiently varying counterpart (equation 5.7). The velocity field defined by (5.5) and (5.8) is only a weak solution of the steady $2 \mathrm{D}-3 \mathrm{C}$ Euler equations as the absolute value 
function introduces a discontinuity in the axial velocity derivative at $x_{2}=\frac{\pi}{2}+n \pi$, for integer $n$. The presence of viscosity in the full NS simulation will act to smooth out this discontinuity, however, even in the absence of viscosity, the jump in the velocity derivative turns out to be less than $1 \%$ of the value at the vortex core when a separation parameter $\mathrm{C}=0.9$ is used.
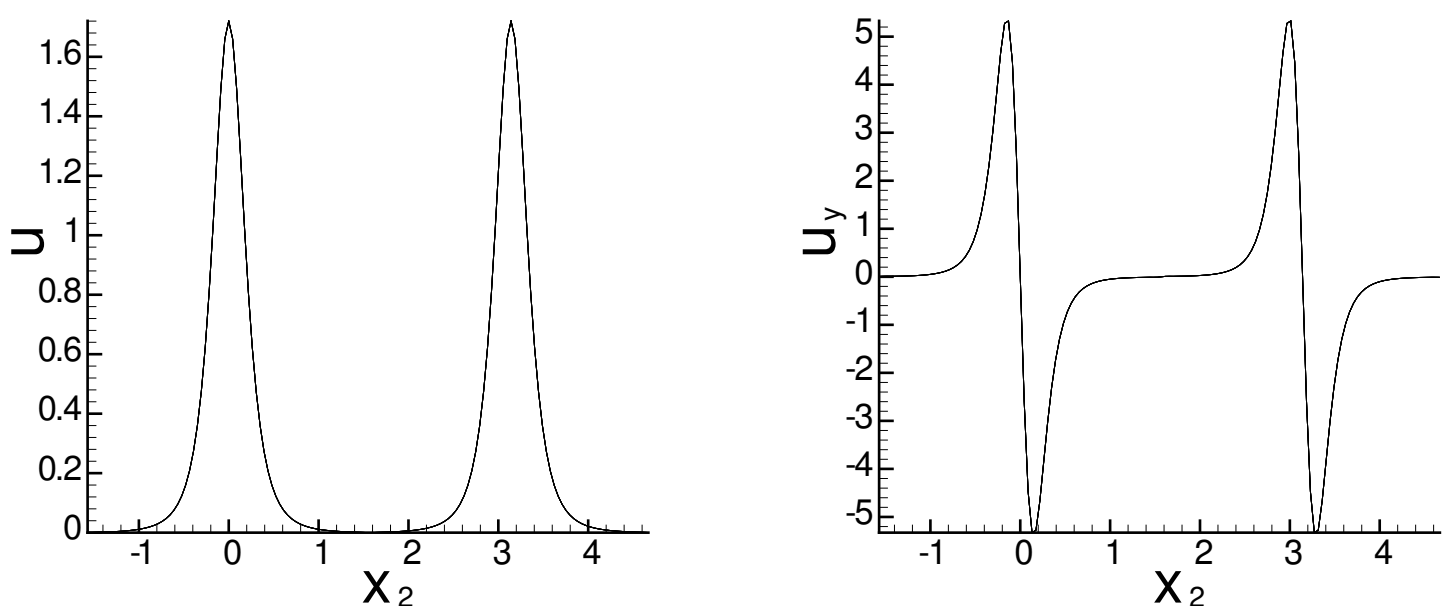

Figure 5.3: Profile of axial velocity and $\frac{\partial u}{\partial y}$ through the core of the vortex array for $\mathrm{c}=0.9$, $\mathrm{q}=1.0$.

\subsubsection{Vortex-Induced Strain Rate}

For an array of point vortices, the strain rate at the center of each vortex is that induced by the array at the vortex position if it were not present. With the separation distance between counter-rotating pairs denoted by $a$, the strain rate can be shown to be $\gamma=\pi \Gamma / 12 a^{2}$. For two isolated point vortices, the strain rate is lower with $\gamma=\Gamma / 2 \pi a^{2}$. Moore and Saffman ${ }^{49}$ calculated that an exterior imposed strain rate distorts the Rankine vortex into an ellipse. When the resulting self-induced strain rate is included ${ }^{40}$ for the counter-rotating pair, this results in a total strain rate at the center of the vortex

$$
\gamma=\Gamma / \pi a^{2}
$$


For the $\mathrm{M} \& \mathrm{M}$ initial condition with no axial flow, the equivalent strain rate at $\left(x_{1}, x_{2}\right)=(0,0)$

$$
\gamma=\pi C \Gamma / 4 a^{2}
$$

Detailed derivations of these results are given in Appendix A. They will be useful later for enabling comparison with isolated vortex pair experiments; however, for now it is interesting to note that for the value $C=0.9$, the strain rate resulting from the M\&M vortex array is approximately twice that felt for the isolated pair.

\subsubsection{Further Code Verification}

The above mentioned initial condition can be used as an albeit crude test for the code by comparing its evolution over one transit time at various Reynolds numbers. In the limit of infinite Reynolds number, the vortices should just rotate and remain identical to the initial condition. The top two plots in Figure 5.4 show the energy vs time at three Reynolds numbers and a comparison between the energy decay rate and the dissipation at the lowest Reynolds number; since it can be shown that on the domain of interest the latter two should be identical for a fully resolved simulation, this can be taken as evidence that the code is performing as expected. The lower two plots show the final flow state at the highest Reynolds number and the maximum difference between the initial and final flow states respectively. As expected the difference decreases with Reynolds number, with the $R e_{\Gamma}=10^{6}$ case being a good approximation to the infinite Reynolds number limit.

In addition to the two-dimensional restriction, the preceding results do not ensure correct implementation of the non-linear terms since they only test the relative magnitudes of the non-linear terms as can be seen from the two-dimensional inviscid vorticity equation,

$$
\frac{\partial \psi}{\partial y} \frac{\partial \omega}{\partial x}-\frac{\partial \psi}{\partial x} \frac{\partial \omega}{\partial y}=0
$$

The absolute magnitude of these terms were tested by comparing term by term with the two-dimensional analytic solution before moving on to more comprehensive three- 

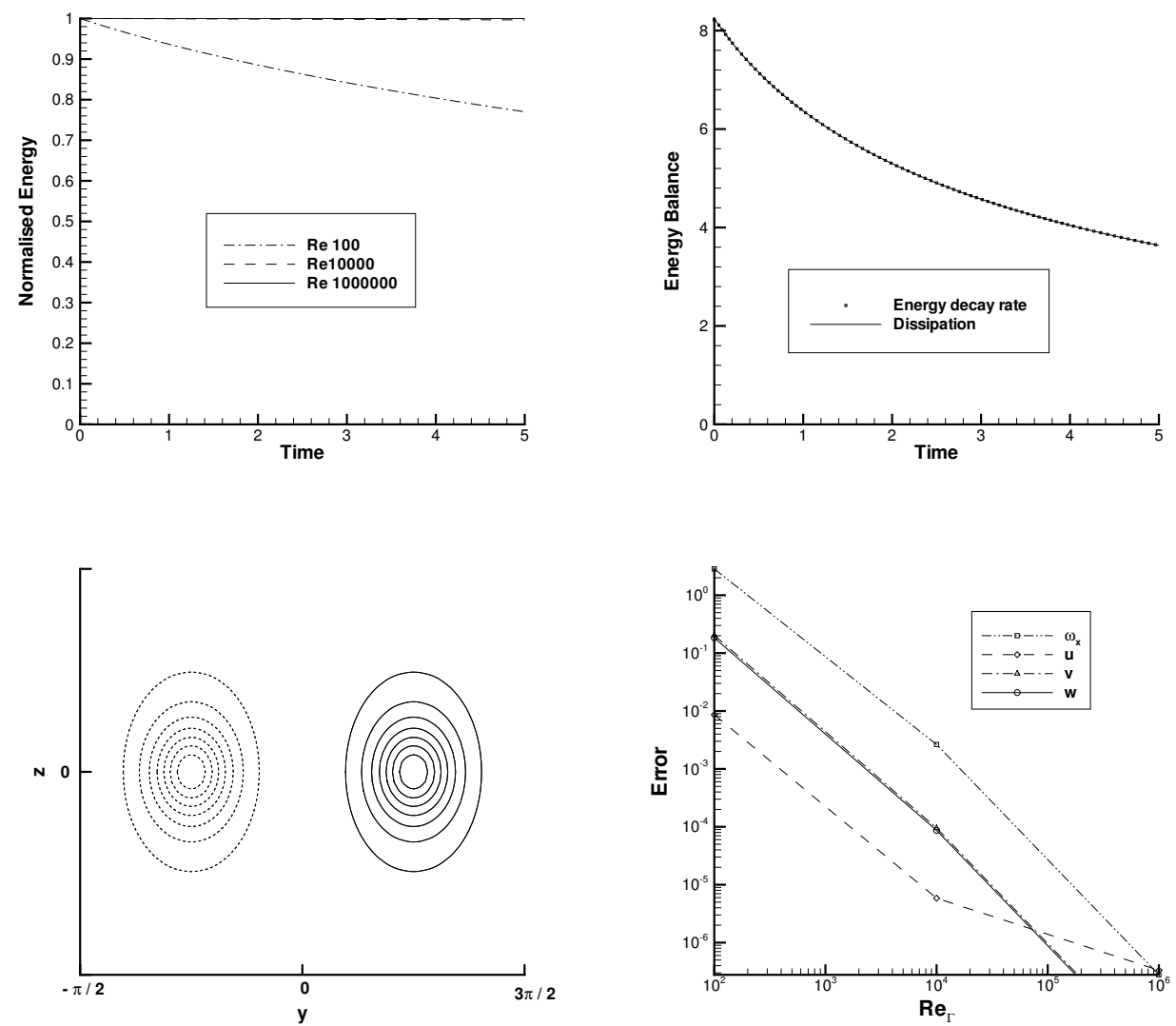

Figure 5.4: Code validation results of simulations of the Mallier and Maslowe vortex array with superimposed axial velocity. Energy evolution at 3 different $R e_{\Gamma}$ (top left), energy decay rate compared to dissipation (top right), the flow field after one transit time at $R e_{\Gamma}=10^{6}$ (bottom left), and the maximum difference between the flow field after one transit time and the initial condition.

dimensional simulations.

Julien et al. ${ }^{28}$ studied the temporally developing three-dimensional stability of the M\&M vortex array and investigated the growth rates for a range of unstable modes. They found a peak at low wave numbers corresponding to the periodic analogy to the long wavelength Crow instability associated with a counter-rotating vortex pair ${ }^{14}$, as well as a range of unstable modes at higher wave numbers, associated with the elliptic instability of the strained base flow. For $\mathrm{C}=0.9$ the most unstable axial wave number resulting in the Crow type instability mechanism is 0.4 and that perturbations grow in a plane inclined at $\phi=47$ degrees to the $\mathrm{z}$ axis. To replicate this, a simulation 
was performed on a domain comprising one axial wavelength (wave number $\mathrm{k}$ ) with the base $\mathrm{M} \& \mathrm{M}$ vorticity distributions perturbed with amplitude $\mathrm{A}$ according to

$$
\omega_{x}(x, y, z)=\omega_{m \& m}\left(y^{\prime}, z^{\prime}\right)
$$

where

$$
\begin{aligned}
& y^{\prime}=y-A \cos (\phi) \cos (k x), \\
& z^{\prime}=z-A \sin (\phi) \cos (k x) .
\end{aligned}
$$

In order to preserve a divergence free initial vorticity distribution, the remaining components are specified as

$$
\begin{aligned}
& \omega_{y}=-A k \cos (\phi) \sin (k x) \omega_{m \& m}\left(y^{\prime}, z^{\prime}\right), \\
& \omega_{z}=-A k \sin (\phi) \sin (k x) \omega_{m \& m}\left(y^{\prime}, z^{\prime}\right) .
\end{aligned}
$$

Simulations were performed at grid resolutions of 32x96x64, 48x128x128 and $72 \times 256 \times 256$ for $R e_{\Gamma}=100$ over a period of between 4 and 6 vortex transit times. It is believed that the highest resolution simulation captures all scales of the resulting motion.

Flow visualization through an isosurface of vorticity in Figure 5.5 shows the development of the periodic analogy to the Crow instability. Figure 5.6 shows that grid convergence is obtained in the decay of both energy and enstrophy and that the perturbation energy decay rate is in good agreement with linear theory. Grid convergence is once again demonstrated in Figure 5.7 whereby contours of vorticity after four transit times are plotted on top of each other at three locations. Finally, Fourier coefficients were compared directly to ensure that grid convergence was obtained. 

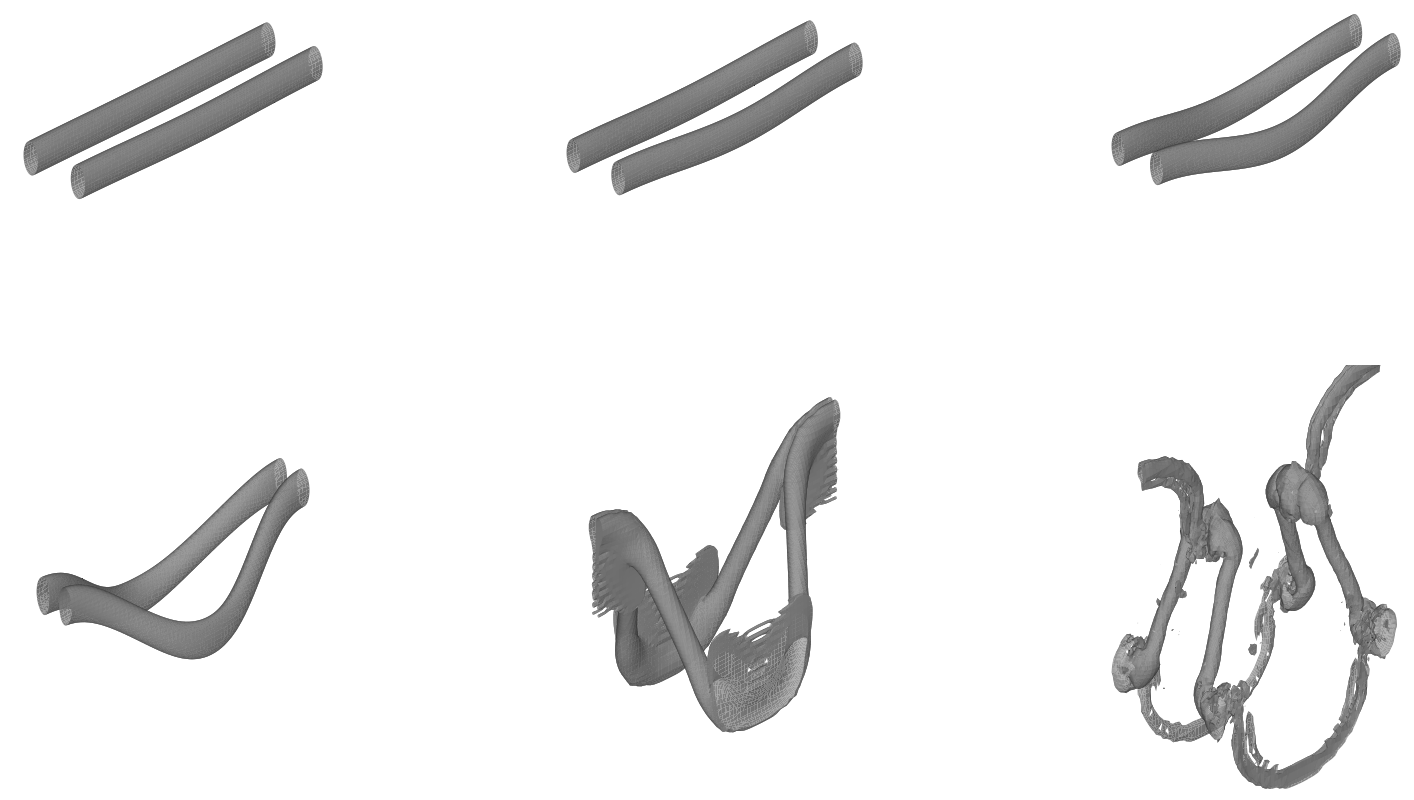

Figure 5.5: Flow visualization of the periodic Crow instability using 1 vorticity isosurface.
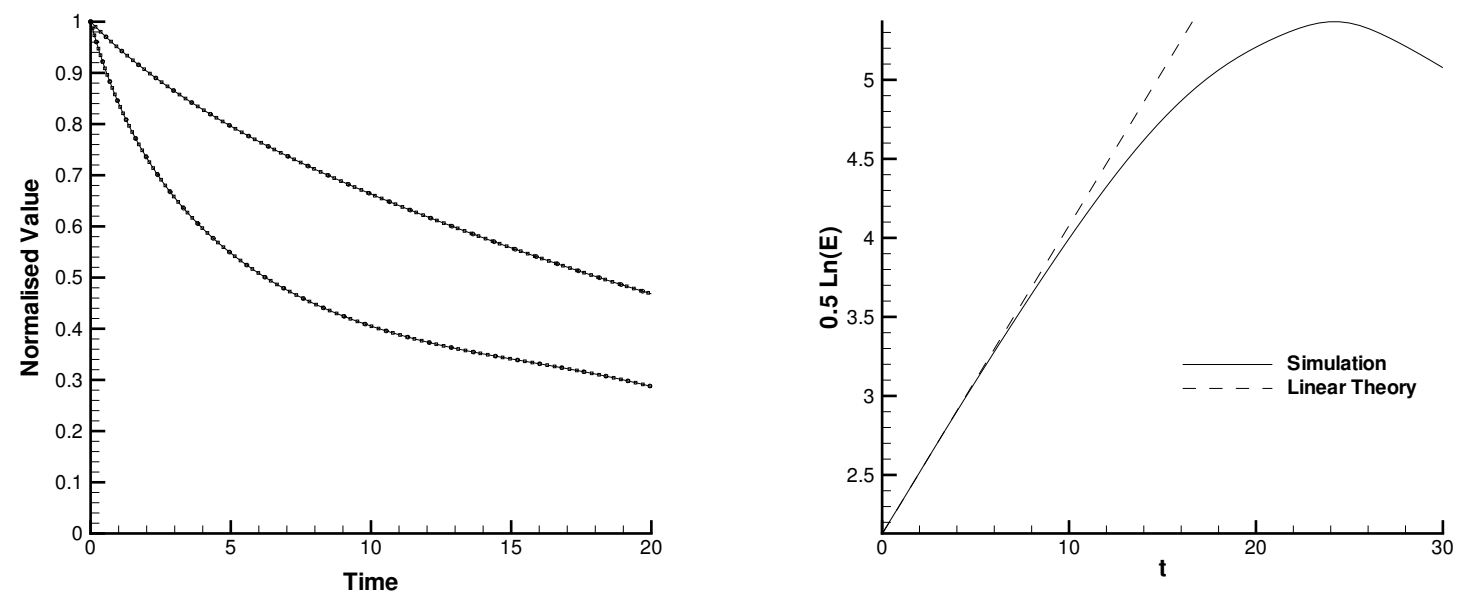

Figure 5.6: (a) Effect of grid resolution on energy (top) and enstrophy (bottom). Solid line $=72 \times 256 \times 256$, Squares $=48 \times 128 \times 128$, Circles $=32 \times 96 \times 64$. (b) Perturbation growth rate for the three-dimensional simulation compared with the linear analysis of Julien et al. 28 . 

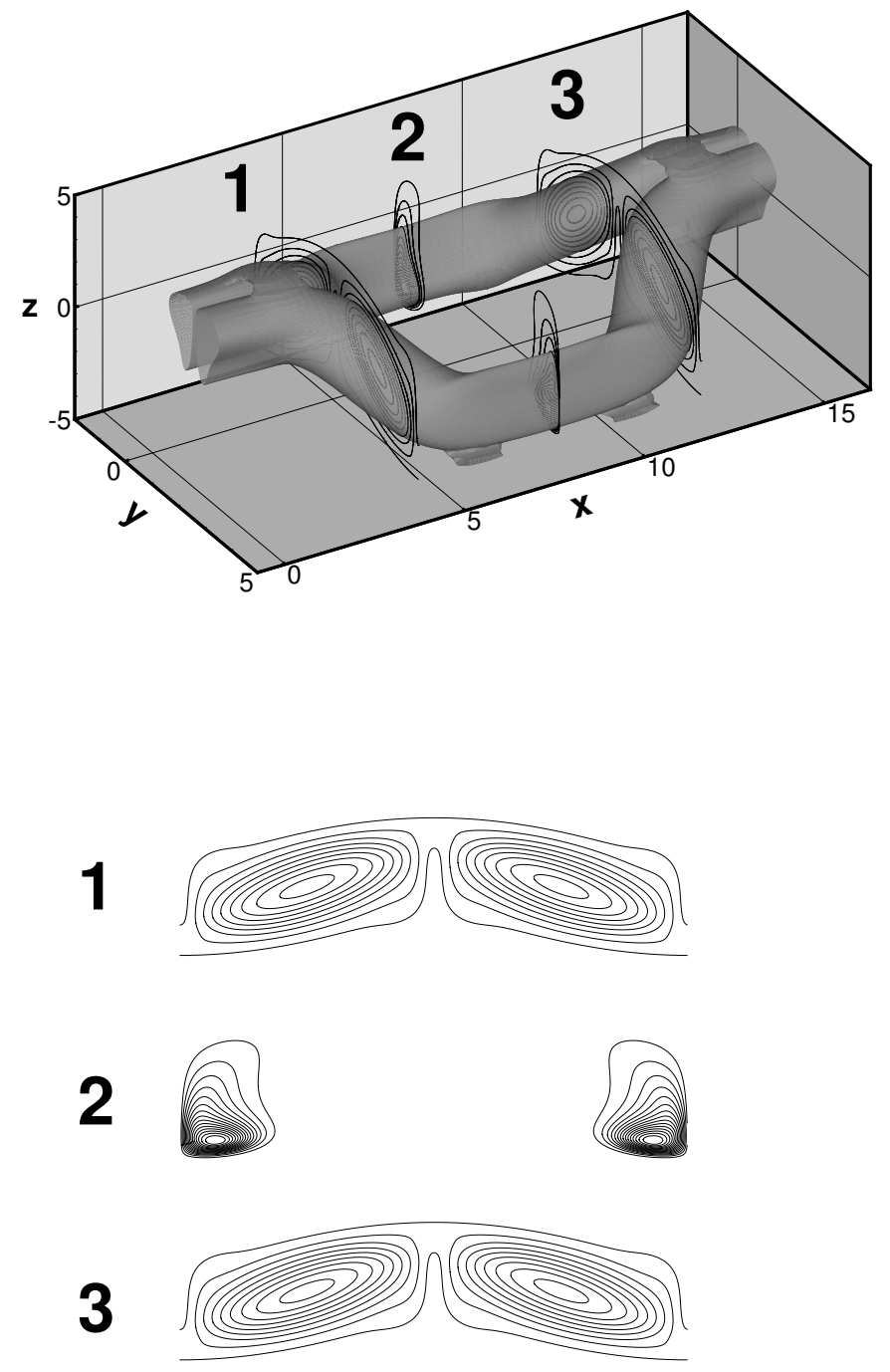

Figure 5.7: Contours of vorticity at the two highest resolutions are layered on top of each other (right) at 3 locations (left) demonstrating grid convergence. 


\section{Chapter 6}

\section{Stability of the M \& M Vortex Array}

Julien et al. ${ }^{28}$ (JC henceforth) studied the linear stability of the M\&M vortex without axial flow, finding periodic array analogies to the long and short-wavelength modes that Crow ${ }^{14}$ and Widnall et al. ${ }^{76}$, respectively found for the two vortex system. They found that, while the long wavelength Crow instability is almost independent of $R e$, viscous action damps the shorter-wavelength modes. Stability analysis of the $\mathrm{BV}^{36}$ shows that the presence of axial flow introduces helical mode instabilities for sufficiently low $q$. To study the effect of axial flow on the stability of the vortex array, we

utilize the NS equations linearized with respect to perturbations in velocity $u_{i}^{\prime}$ and pressure $P^{\prime}$ about the base flow $u_{i}$ defined by (5.5) and (5.8).

$$
\begin{aligned}
\frac{\partial u_{j}^{\prime}}{\partial x_{j}} & =0 \\
\frac{\partial u_{i}^{\prime}}{\partial t}+u_{j}^{\prime} \frac{\partial u_{i}}{\partial x_{j}}+u_{j} \frac{\partial u_{i}^{\prime}}{\partial x_{j}} & =-\frac{\partial P^{\prime}}{\partial x_{i}}+\frac{1}{R e} \frac{\partial^{2} u_{i}^{\prime}}{\partial x_{j}^{2}}
\end{aligned}
$$

The perturbation field can be written in the form

$$
\left[u_{i}^{\prime}, P^{\prime}\right]\left(x_{1}, x_{2}, x_{3}, t\right)=\sum_{k_{1}}\left[\hat{u}_{i}{ }^{\prime}, \hat{P}^{\prime}\right]\left(k_{1}, x_{2}, x_{3}, t\right) e^{i k_{1} x_{1}}
$$

Since the base flow is uniform in the $x_{1}$ direction, (6.2) causes (6.1) to de-couple in axial wave space, allowing the stability problem to be solved independently at each $k_{1}$ simultaneously. Despite this simplification, however, the non-uniform nature of the 
base flow in the $x_{2}-x_{3}$ plane makes a full modal analysis difficult to perform. For this reason, we follow a similar numerical procedure to JC which finds only the most unstable wave-mode at each axial wavelength. Equation (6.1) was integrated as an initial-value problem using the same parallelization, mapping and splitting methods used for the non-linear code. The instability modes are separated into two families, namely the sinuous mode (anti-symmetric) defined by

$$
\left[u_{1}^{\prime}, u_{2}^{\prime}, u_{3}^{\prime}\right]\left(x_{2}, x_{3}, t\right)=\left[-u_{1},-u_{2}, u_{3}\right]\left(x_{1}, x_{2}+\pi,-x_{3}, t\right)
$$

and the varicose mode (symmetric) by

$$
\left[u_{1}^{\prime}, u_{2}^{\prime}, u_{3}^{\prime}\right]\left(x_{2}, x_{3}, t\right)=\left[u_{1}, u_{2},-u_{3}\right]\left(x_{1}, x_{2}+\pi,-x_{3}, t\right)
$$

The initial condition for the divergence free perturbation velocity field comprises white noise exhibiting each of these symmetry properties, modulated by an exponential decay in $x_{3}$.

At each time step the kinetic energy at each axial wavelength $k_{1}$ is computed and the perturbation energy E determined by integrating over the $x_{2}-x_{3}$ plane. The integration is carried on in time sufficiently long for the most unstable eigenmode to dominate, as evidenced by convergence of the growth rate defined as ${ }^{28}$

$$
\sigma=\lim _{t \rightarrow \infty} \frac{1}{2} \frac{d \ln E}{d t}
$$

Figure 6.1 shows the maximum growth rates that result in the absence of axial flow at $R e=\Gamma / 2 \pi \nu=20,000$. For sinuous modes two regimes are identified, separated by a region of negligibly small growth rate. The first of these occurs for $k_{1}<1$ and corresponds to the periodic analogy to the long-wavelength Crow instability mode $(\mathrm{CM})$ with a peak at $k_{1} \approx 0.4$. The second, for $k_{1}>2$, is associated with the shortwavelength Widnall-like instability modes (WM) and shows two distinct peaks. In contrast, the case of the varicose mode differs from the sinuous in that the imposed symmetries don't allow the Crow instability to develop. The corresponding growth 


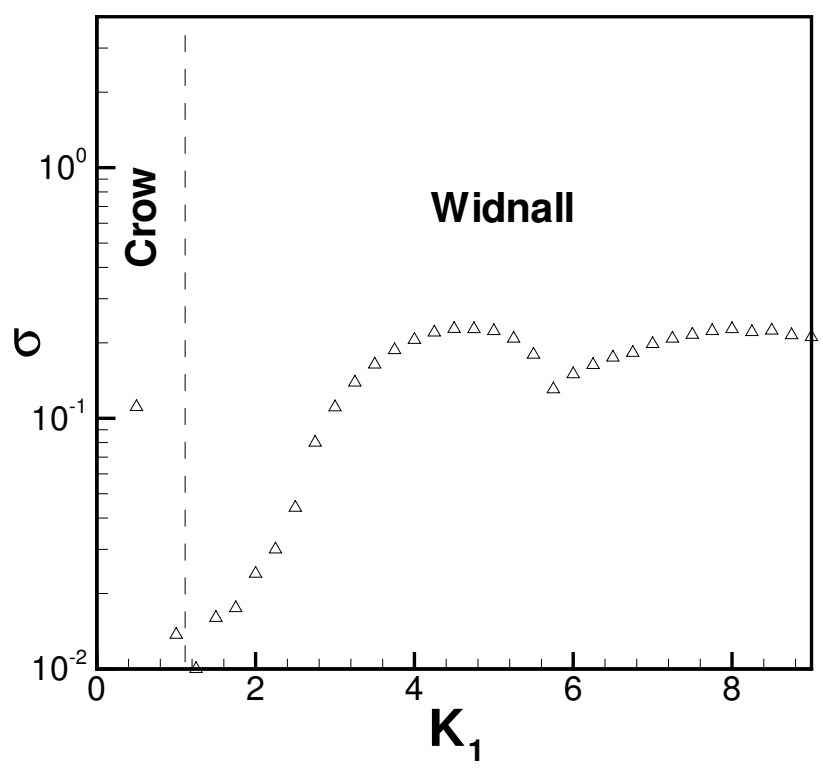

(a)

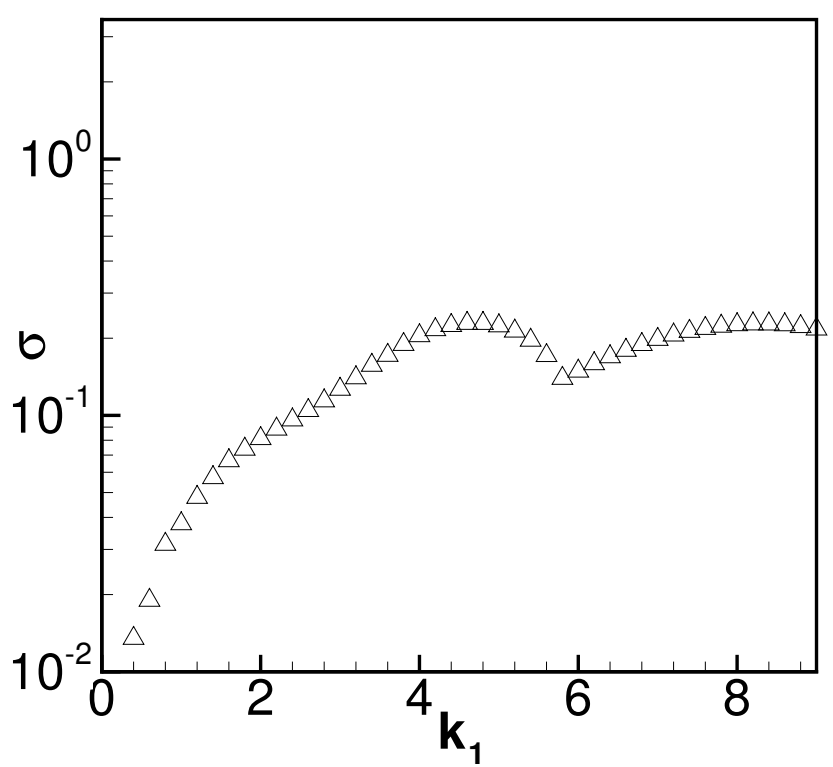

(b)

Figure 6.1: Growth rate of the anti-symmetric modes (top) with no axial velocity $\left(q_{0}=\right.$ $\infty)$, showing the Crow Instability regime at small wavenumbers and the short-wavelength Widnall like modes at high wavenumbers. The symmetric modes (bottom) are similar at high wavenumbers, but are not susceptible to the Crow modes. 
rates in this region are very low. At toward shorter wavelengths, they approach those for the previous case; moderate growth rates being observed in the region considered stable to sinuous modes.

The effect of Reynolds number is investigated in Figure 6.2 showing the maximum growth rates for sinuous modes at discrete axial wave numbers for $q=\infty$, which corresponds to no axial flow. Simulations at $R e=20,000$ and 5,000 are shown from

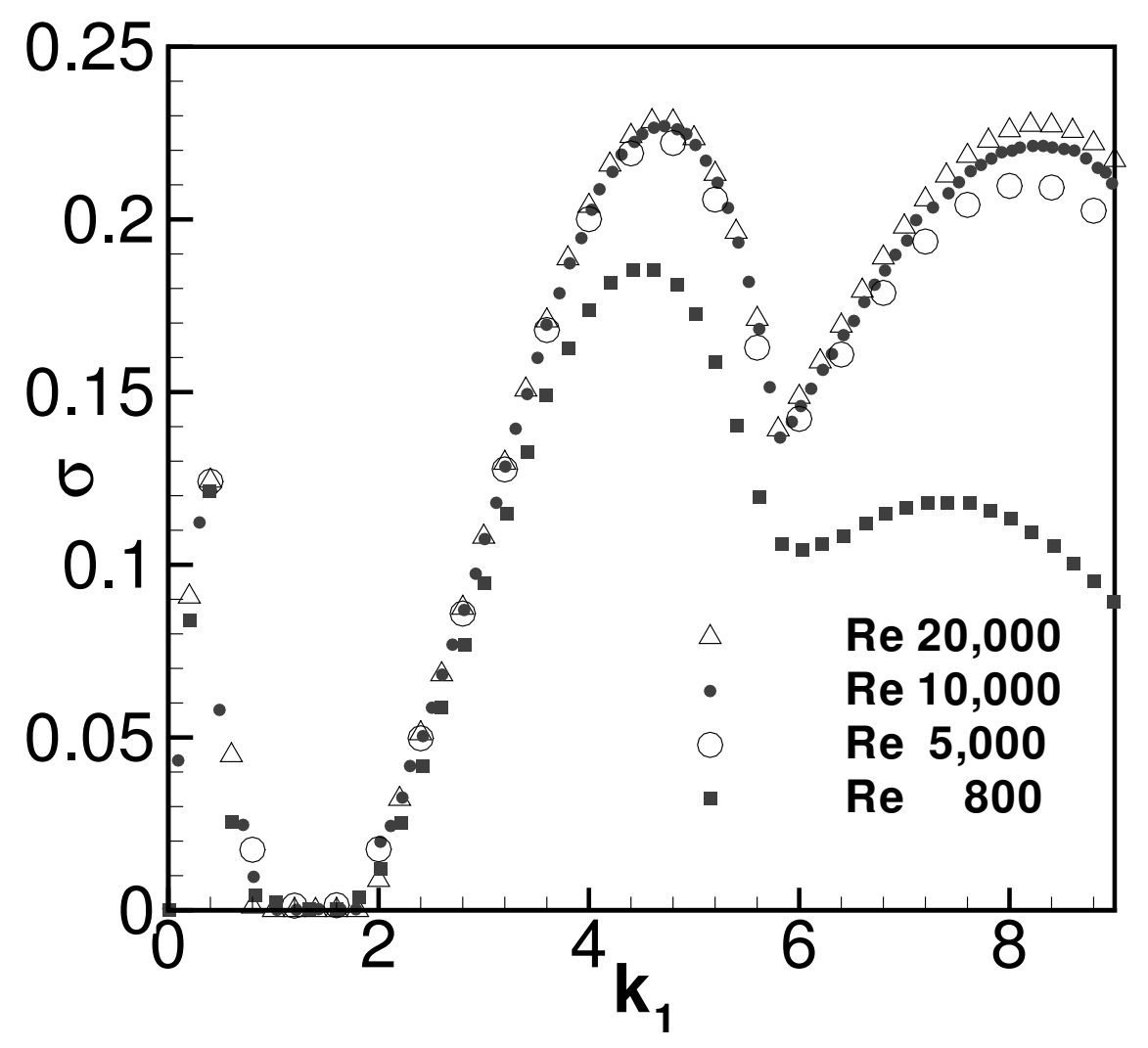

Figure 6.2: Growth rate of the anti-symmetric modes with no axial velocity $\left(q_{0}=\infty\right)$, showing once again the two regimes. The Crow instability at long wavelengths and the more viscous dependent Widnall instabilities at shorter wavelengths. Filled symbols are taken from the work of Julien et al. ${ }^{28}$, at $R e=\Gamma / 2 \pi \nu$ of 800 and 10,000. Open symbols are computed from the present study at $R e=5,000$ and 20,000.

the present study with data points at 800 and 10,000 being taken from the work of JC. Our results can be seen to support the findings of JC that the peak CM 
appears to be viscosity independent, while the WM becomes increasingly effected at higher wavenumber. Additionally, the good agreement at the higher Reynolds number provides further evidence that the (linearized) code is performing as expected.

We can therefore hypothesize that since viscosity effects are confined to high wave numbers, Re will influence this family of instabilities in the same manner. Hence, we conclude that, since little difference is found between the three highest Reynolds numbers, the $R e=20,000$ case is a good approximation to the limit $R e \rightarrow \infty$. This $R e$ is also of interest as it is near that estimated for the mixing transition to occur ${ }^{16}$.

Figure 6.3 shows the stability properties when an axial flow, $q_{0} \neq \infty$, is added to the base flow. It can be seen that the resulting vortex array exhibits larger growth

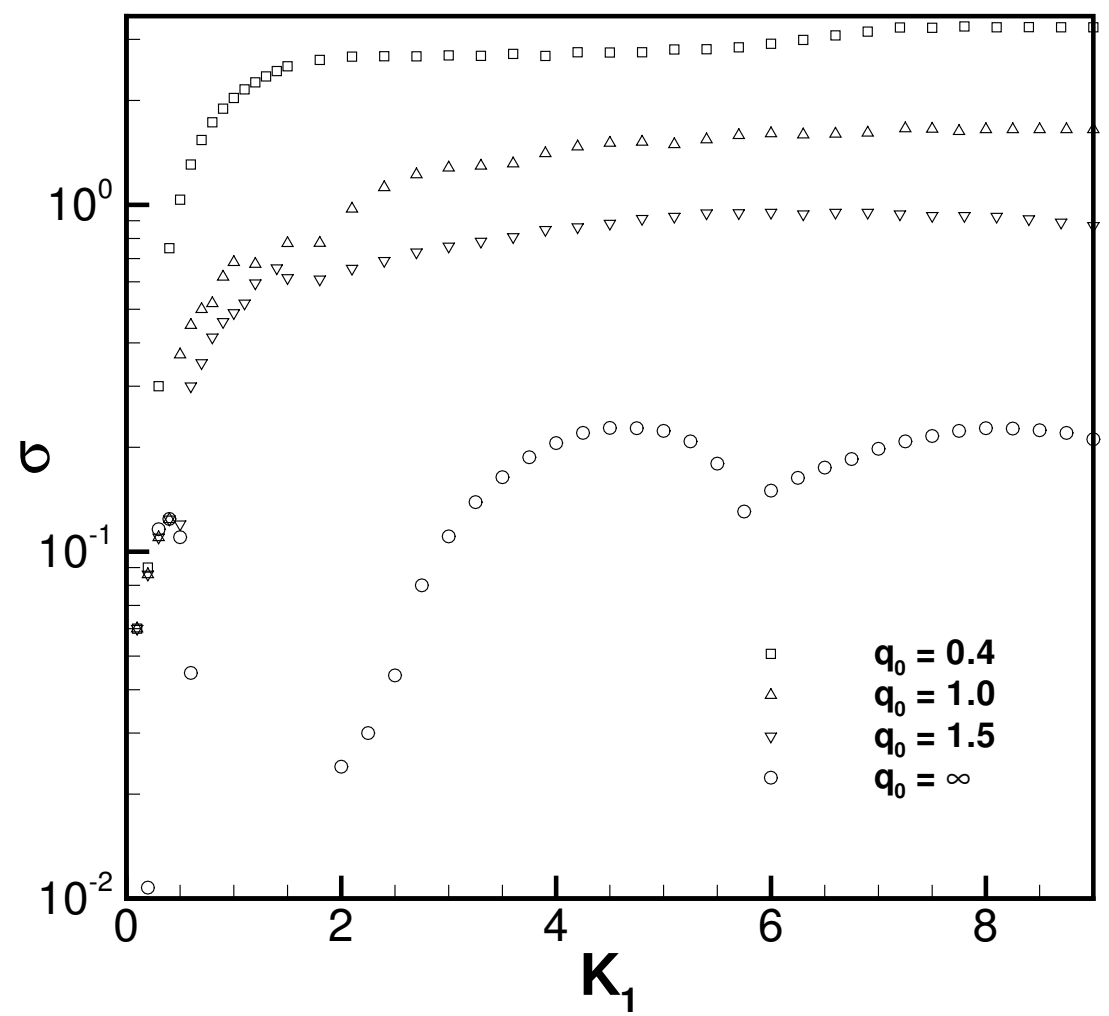

Figure 6.3: Growth rate vs axial wavenumber for the anti-symmetric modes of the M\&M vortex with $\mathrm{C}=0.9$ for different values of $q_{0}$. 
rates due to the additional instability mechanism resulting from the axial flow. As the magnitude of the axial velocity is increased (corresponding to a decrease in $q_{0}$ ) the maximum growth rates increase significantly. Of particular note for the sinuous mode is the point $k_{1} \approx 0.4, \sigma \approx 0.1$, which is in the vicinity of the peak growth rate of the Crow Instability. Of the four cases considered, only for the highest axial velocity does the most unstable mode cease to be associated with the Crow instability. Conversely, at higher wave numbers the largest growth rates increase with decreasing q and are presumably the periodic analog of the helical instability modes observed for the BV. Finally, it should be noted that the axial velocity related modes exhibit high growth in the region $1<k_{1}<2$ where insignificant growth of the sinuous modes was observed in the absence of axial flow.

We note finally that the most unstable linear eigen-mode associated with each axial wavelength could be determined from the numerical procedure. One of the main purposes of this stability study, however, was to determine the axial domain size necessary to capture in a Direct Numerical Simulation, the most unstable long wavelength mode. We now move on to the DNS, and defer a discussion of these mode shapes until the more general analysis of the non-linear evolution of the base state. 


\section{Chapter 7}

\section{D-3C Direct Numerical Simulation}

\subsection{Diagnostics}

The study of the vortex array is now extended beyond the linear analysis, by considering the evolution of the 3D-3C Navier Stokes equations. To aid in the analysis of the DNS and LES presented subsequently, we develop diagnostics for analyzing the flow. We begin by defining by $\langle\phi\rangle$ the integral of the quantity $\phi$ over the domain

$$
\langle\phi\rangle=\int_{0}^{L_{x_{1}}} \int_{0}^{2 \pi} \int_{-\infty}^{\infty} \phi\left(x_{1}, x_{2}, x_{3}\right) d x_{1} d x_{2} d x_{3} .
$$

For all $\phi$ considered presently, this integral exists. Taking the dot product of (5.1) with $u_{i}$, and integrating over the domain using $x_{1}, x_{2}$ periodicity as well as quiescent flow at infinity, a conservation equation for the volume averaged kinetic energy $\langle E\rangle$ is obtained

$$
\frac{d}{d t}\left\langle\frac{1}{2} u_{i}^{2}\right\rangle=-\frac{2}{R e}\left\langle S_{i j} S_{i j}\right\rangle,
$$

where $S_{i j}$ is the rate of strain tensor and the term on the right represents the viscous dissipation $\left\langle\epsilon_{\nu}\right\rangle$ which can be written

$$
<\epsilon_{\nu}>=\frac{1}{R e}\left\langle\left(\frac{\partial u_{i}}{\partial x_{j}}\right)^{2}\right\rangle .
$$


The enstrophy defined as $\omega^{2}$ is a measure of the turbulence intensity and can be shown to be equal to

$$
\omega_{i} \omega_{i}=\left(\frac{\partial u_{k}}{\partial x_{j}}\right)^{2}-\frac{\partial u_{k}}{\partial x_{j}} \frac{\partial u_{j}}{\partial x_{k}} .
$$

Applying (7.1) to (7.4) it can be seen that the volume averaged enstrophy is proportional to the dissipation and hence also, a measure of the turbulence intensity.

$$
<w_{i} w_{i}>=<\left(\frac{\partial u_{k}}{\partial x_{j}}\right)^{2}>=R e<\epsilon_{\nu}>.
$$

This is useful when considering LES, where only the resolved-scale enstrophy integral can be calculated. Both resolved and subgrid dissipation integrals can be combined, however, providing a means to estimate the total enstrophy or alternatively be interpreted directly as a measure of the turbulence intensity of the flow. Finally, as a measure of the intensity of each vortex, we define by $\bar{\Gamma}$ the circulation around one positively signed vortex in the array, averaged in the axial direction

$$
\bar{\Gamma}=\frac{1}{L_{x_{1}}} \int_{0}^{L_{x_{1}}} \int_{-\pi / 2}^{\pi / 2} \int_{-\infty}^{\infty} \omega\left(x_{1}, x_{2}, x_{3}\right) d x_{3} d x_{2} d x_{1} .
$$

Two techniques are used for flow visualization, the first of which is based on the local velocity field around a critical point ${ }^{12}$, which to linear order is governed by the velocity gradient tensor $\partial u_{i} / \partial x_{j}$. For an incompressible flow, the eigenvalues $\lambda$ of $\partial u_{i} / \partial x_{j}$ are calculated from

$$
\lambda^{3}+Q \lambda+R=0
$$

where

$$
Q=-\frac{1}{2}\left[S_{i j} S_{j i}+\Omega_{i j} \Omega_{j i}\right]
$$

and

$$
R=-\frac{1}{3}\left[S_{i j} S_{j k} S_{k i}+3 \Omega_{i j} \Omega_{j k} S_{k i}\right]
$$

are the second and third invariants of $\partial u_{i} / \partial x_{j}, S_{i j}$ and $\Omega_{i j}$ being the symmetric and antisymmetric parts of the velocity gradient tensor respectively. $Q>0$ implies a pointwise dominance of rotation over strain magnitude and hence provides a means 
to identify vortical structures ${ }^{12,24}$. The present base flow has strong background rotation in vortices, and hence is characterized by mostly positive values of Q. For this reason iso-surfaces at discrete $Q>0$ are chosen to provide visualization of the resulting structures. Further, this strong base rotation is the reason more sophisticated techniques incorporating pressure minima (due to our base flow the pressure should be lower than ambient everywhere), which have been successfully used for detecting structures in box turbulence ${ }^{26,33}$, were not needed. The second means of visualizing the flow is through the passive scalar concentration $c$. The initial condition for $c$ takes the same form as the vorticity in (5.4), and is intended to be representative of the diffusion of smoke or die injected down the core of each vortex. We choose $S c=1$ so that no extra resolution requirements are imposed on the DNS.

\subsection{Direct Numerical Simulation Results}

A Direct Numerical Simulation (DNS) of the M\&M vortex both with and without axial flow was performed at $R e \equiv \Gamma^{*} / 2 \pi \nu=1000$. The axial extent of the domain is set to contain a single wavelength of the most unstable Crow mode. Each vortex in the array (i.e. the half domain) is resolved with $64 \times 64 \times 128$ points, the initial core being described by 24 points in the diameter. The base flow is perturbed with divergence-free random noise exponentially decaying with $x_{3}$, and the NS equations integrated in time. The time step is chosen such that the initial CFL number is 0.1. The various flow regimes resulting throughout the development of both the $q_{0}=\infty$ and $q_{0}=1$ initial conditions are analyzed by considering the evolution of the integrated quantities $\langle E\rangle,\langle\epsilon\rangle$ and $\bar{\Gamma}$ in Figures 7.1(a-b) as well as through snapshots

of the flow at several stages in Figure 7.2. Visualization is through an isosurface of $Q=1$ shaded by local dissipation $\epsilon$.

\subsubsection{Early development}

Consistent with the linear stability study, Figure 7.2(b) shows that the destabilizing effect of axial flow causes the initial perturbations to grow at a faster rate than in 


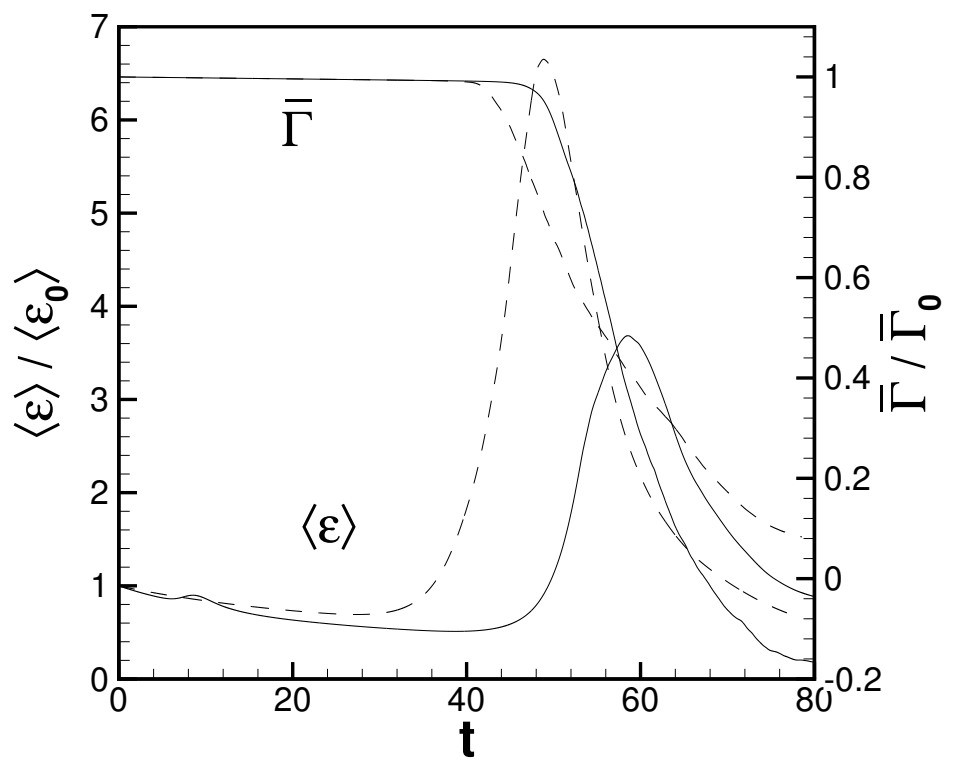

(a)

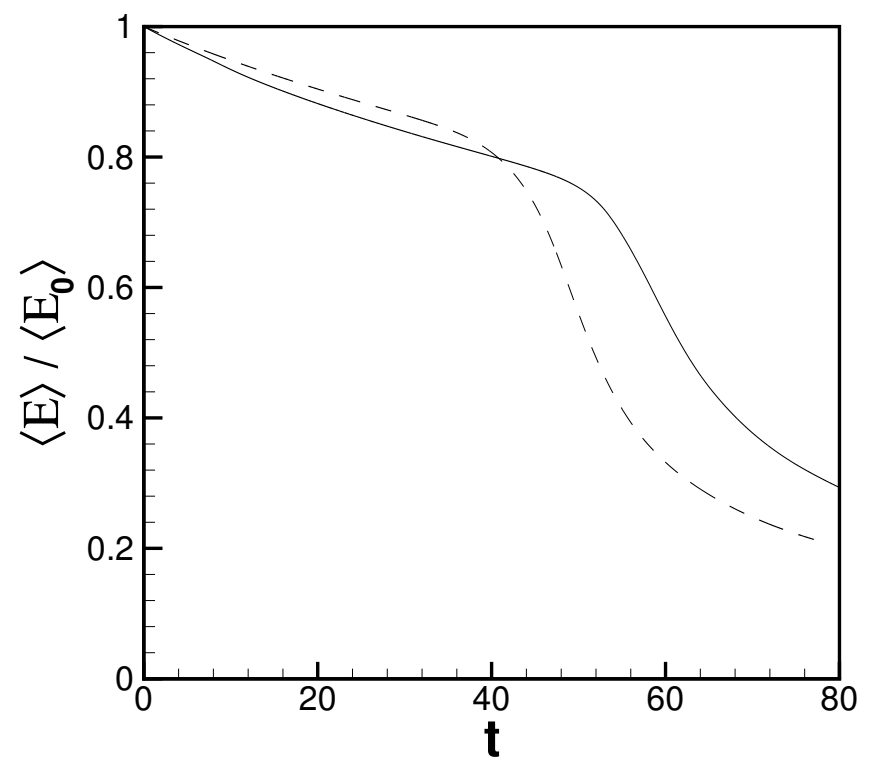

(b)

Figure 7.1: Evolution of volume integrated quantities for the DNS of the $R e=\Gamma / 2 \pi \nu=$ 1000 M\&M vortex with $C=0.9$ for $q_{0}=1$ (solid lines) and $q_{0}=\infty$ (dashed lines). (a) dissipation and circulation (b) normalized energy. 
its absence; helical structures with wavenumber $k_{x} \approx 2$ accompanied by regions of increased dissipation are observed to develop on each vortex in the array. The linear stability study found a local maximum in the growth-rate vs axial wave number curve (Figure 6.3) at around this wavelength; Viscosity most likely damps the higher growth rate, shorter wavelength modes in the viscous DNS. In Figure 7.1(a) it can be seen that globally, this growth of the initial perturbations leads to a slight increase in the volume averaged dissipation and a corresponding faster decay of kinetic energy. As expected, the averaged circulation is unaffected by the axial velocity since its destabilizing effect acts on each vortex independently and does not lead to enhanced inter-vortex vorticity convection. During this initial phase, the evolution of each vortex is qualitatively similar to that of an isolated vortex, and is best described in cylindrical components. Figure 7.3 shows that, similarly to the isolated case, the maximum axial velocity shows a sharp decrease initially while the azimuthal component maintains a constant gradual decay. Presumably, the mechanism is the same as for the Batchelor vortex studied by Jacquin and Pantano ${ }^{25}$, whereby the energy of the axial flow is extracted by turbulence within the core. The perturbations generated are transported outward toward regions of local stability where they don't modify the mainly tangential flow; thus the axial velocity reduces while the angular momentum is maintained. Consequently, $q$ increases and in keeping with the linear analysis the vortex evolves through regimes of progressively weaker instability rates. Ultimately, the core stabilizes with respect to the helical modes and the vortex relaminarizes before the co-operative modes have shown appreciable growth. These phases can clearly be seen in Figure 7.4, which shows contours of vorticity on slices through one vortex in the array.

The early stage was investigated further by performing a DNS at $R e=3,000$ up to time $\mathrm{t}=20$. Using a mesh comprising $128 \times 128 \times 256$ grid points, the simulation represents the highest Reynolds number possible with the available computational resources. Figure 7.5 show axial and azimuthal vorticity contours on slices through one vortex in the array. As expected significantly more small scale motions are generated in the core of the vortex as compared to the lower Reynolds number study. Small 

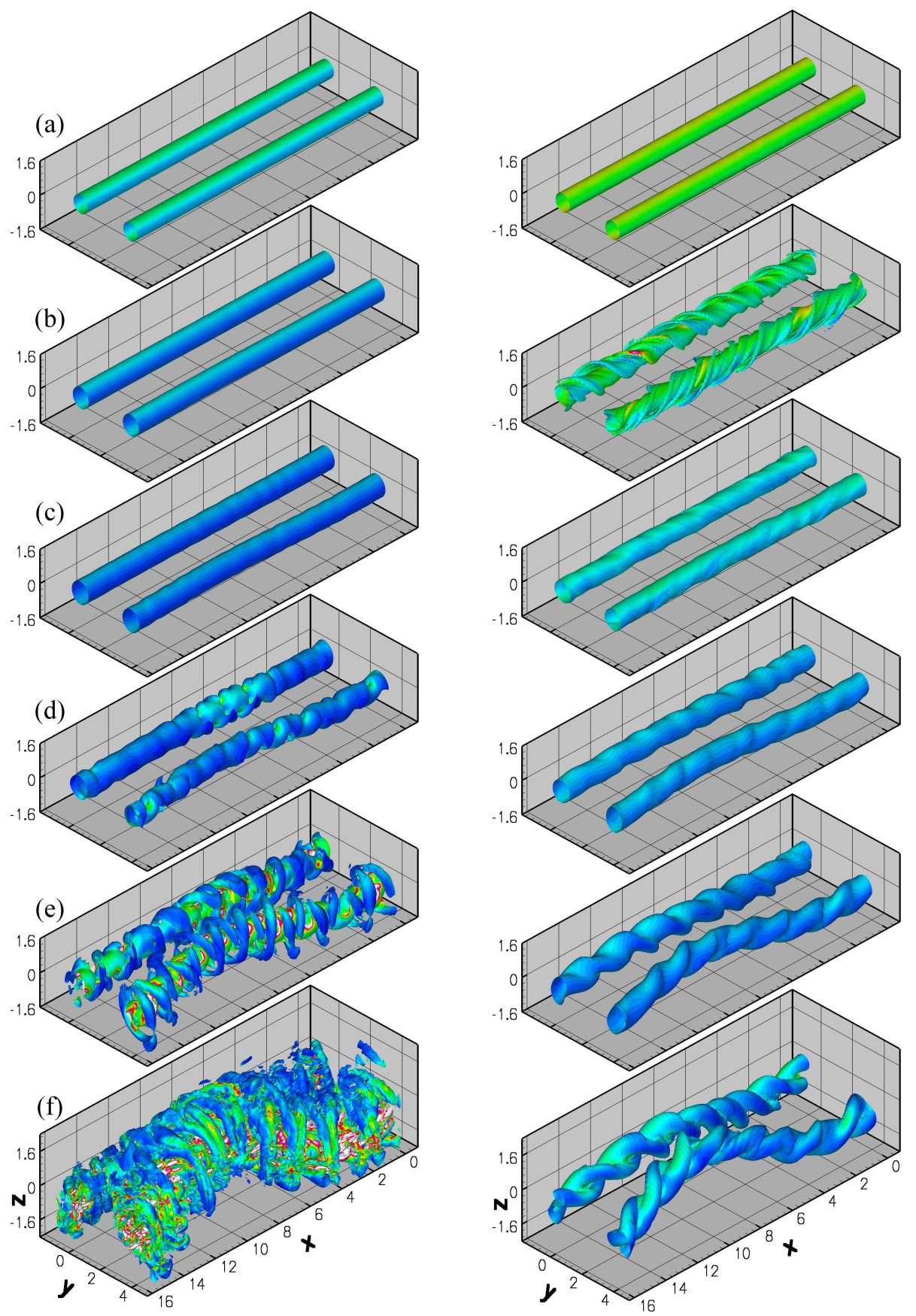

Figure 7.2: Flow visualization comparison through an isosurface with $Q=1$ on which contours of dissipation are plotted for the $R e=\Gamma / 2 \pi \nu=1,000$ DNS of the $\mathrm{C}=0.9 \mathrm{M} \& \mathrm{M}$ vortex with $q_{0}=\infty$ left and $q_{0}=1$ right. Evolution is from top to bottom and shown at non-dimensional times (a) 0 (b) 12 (c) 20 (d) 32 (e) 40 (f) 45. 


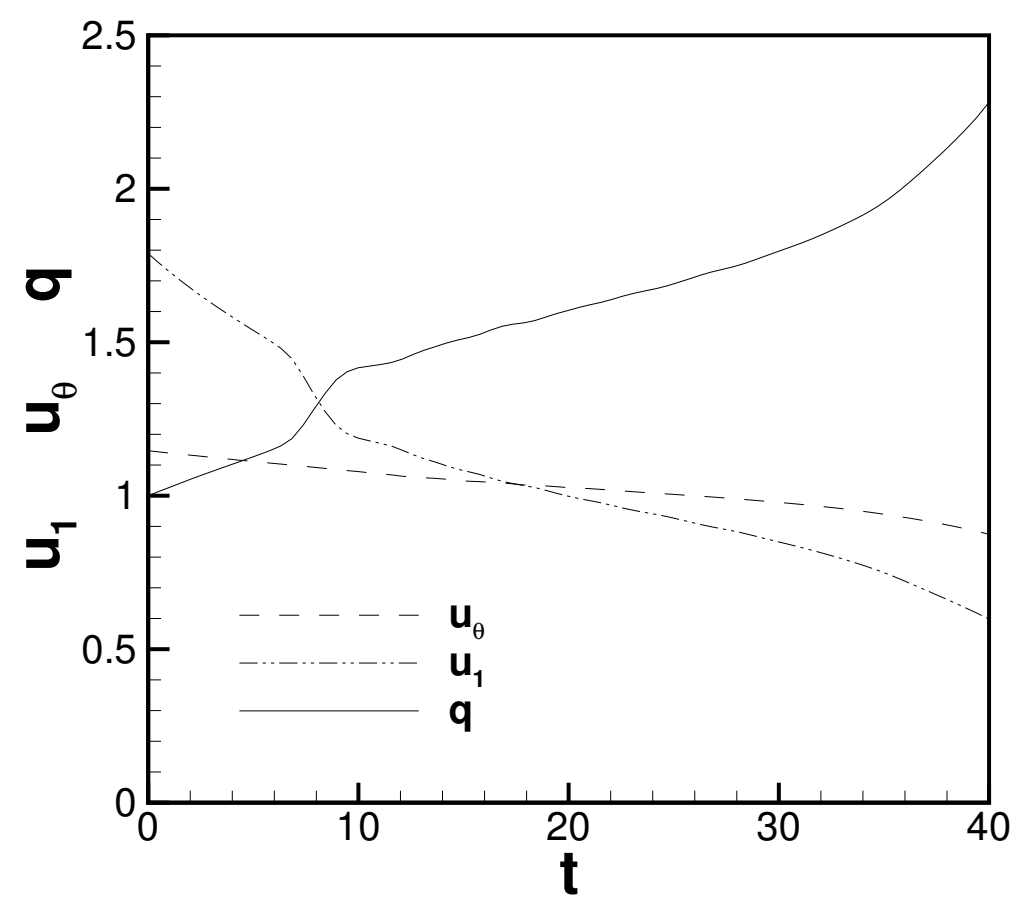

Figure 7.3: Evolution of the maximum axial and azimuthal velocity components as well as the swirl parameter for $\operatorname{Re}=\Gamma / 2 \pi \nu=1000$.

patches of opposite signed vorticity can also be seen embedded in the vortex. These small scale motions are once again seen to branch out from the core into more stable regions where they are damped. By time $\mathrm{t}=20$, the small scale motions have decreased significantly and the axial vorticity contours in the core of the vortex appear to be returning to a cylindrical state. While complete relaminarization by this time was observed in the lower Reynolds number case, the turbulence intensities are still quite high for this simulation. It can be hypothesized that complete relaminarization would eventually occur if this was an isolated vortex, however, the presence of counter rotating pairs create co-operative instabilities, the non-linear effect of which is now considered in detail for the $R e=1000$ simulation. 


\section{1}

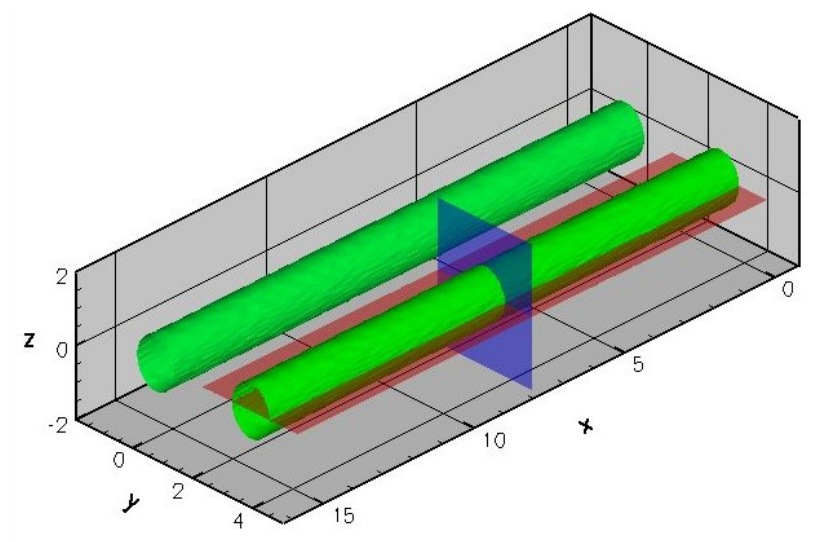

$$
\mathrm{t}=0
$$
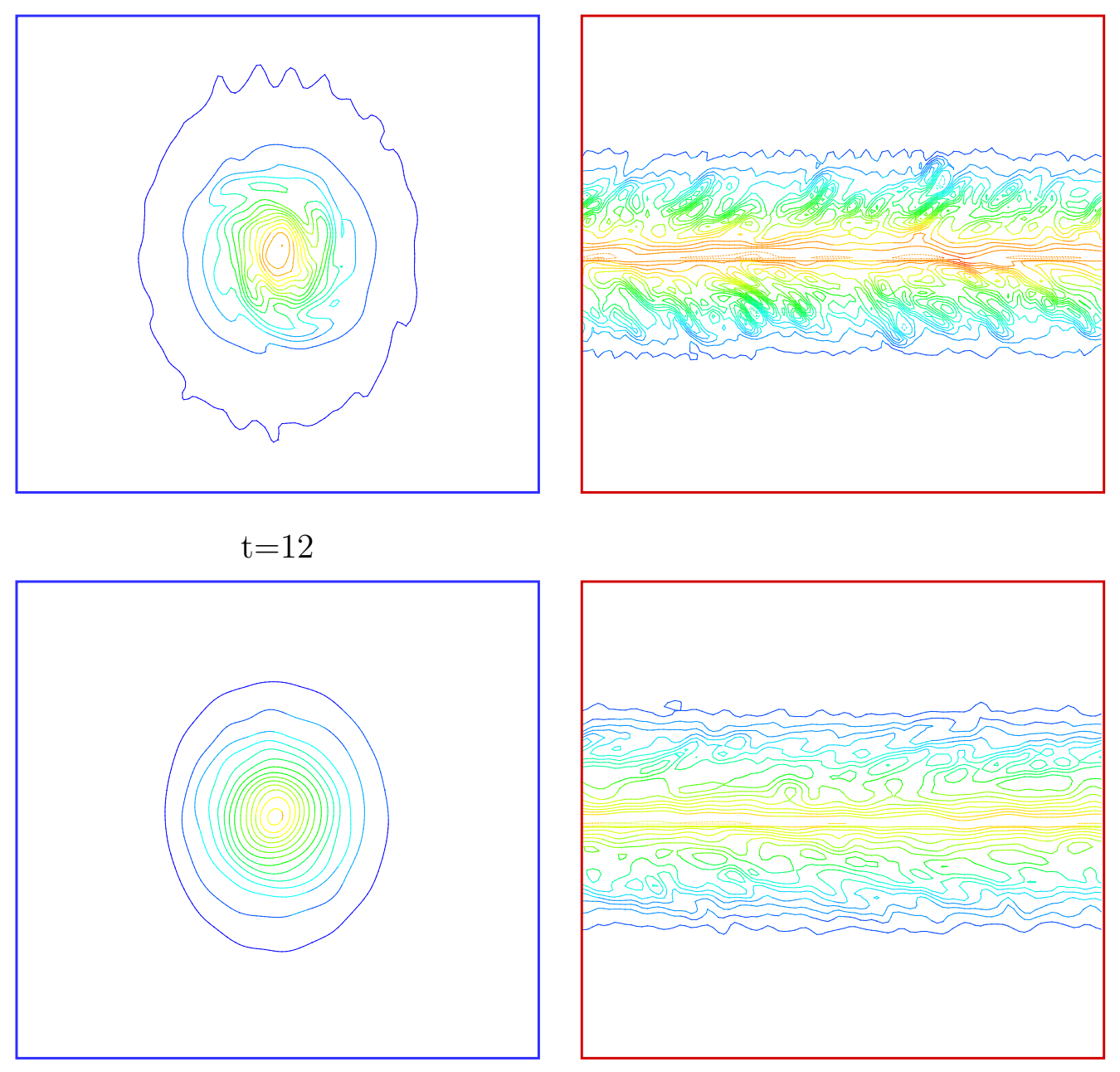

$$
t=20
$$

Figure 7.4: Contours of axial vorticity (left) and azimuthal vorticity (right) colored by axial velocity at time 12 and 20 on slices through one vortex as shown in the top figure. $R e=\Gamma / 2 \pi \nu=1000$. 


\section{2}

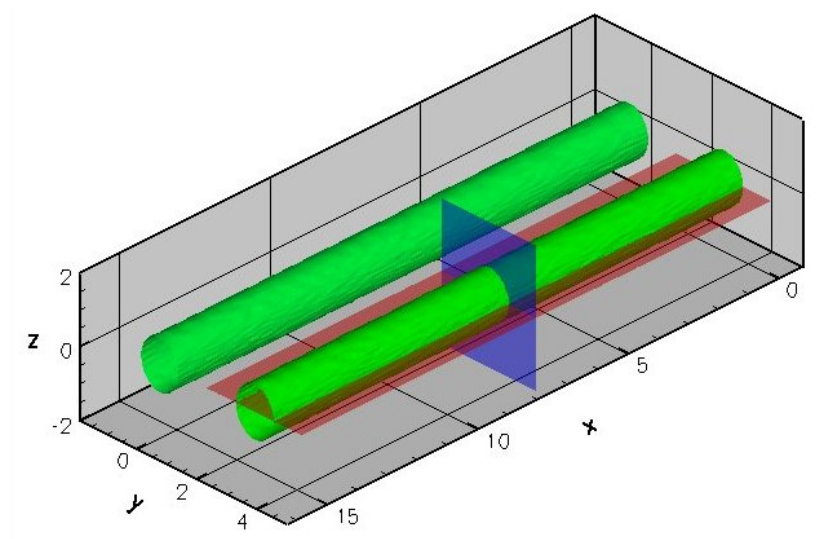

$$
\mathrm{t}=0
$$
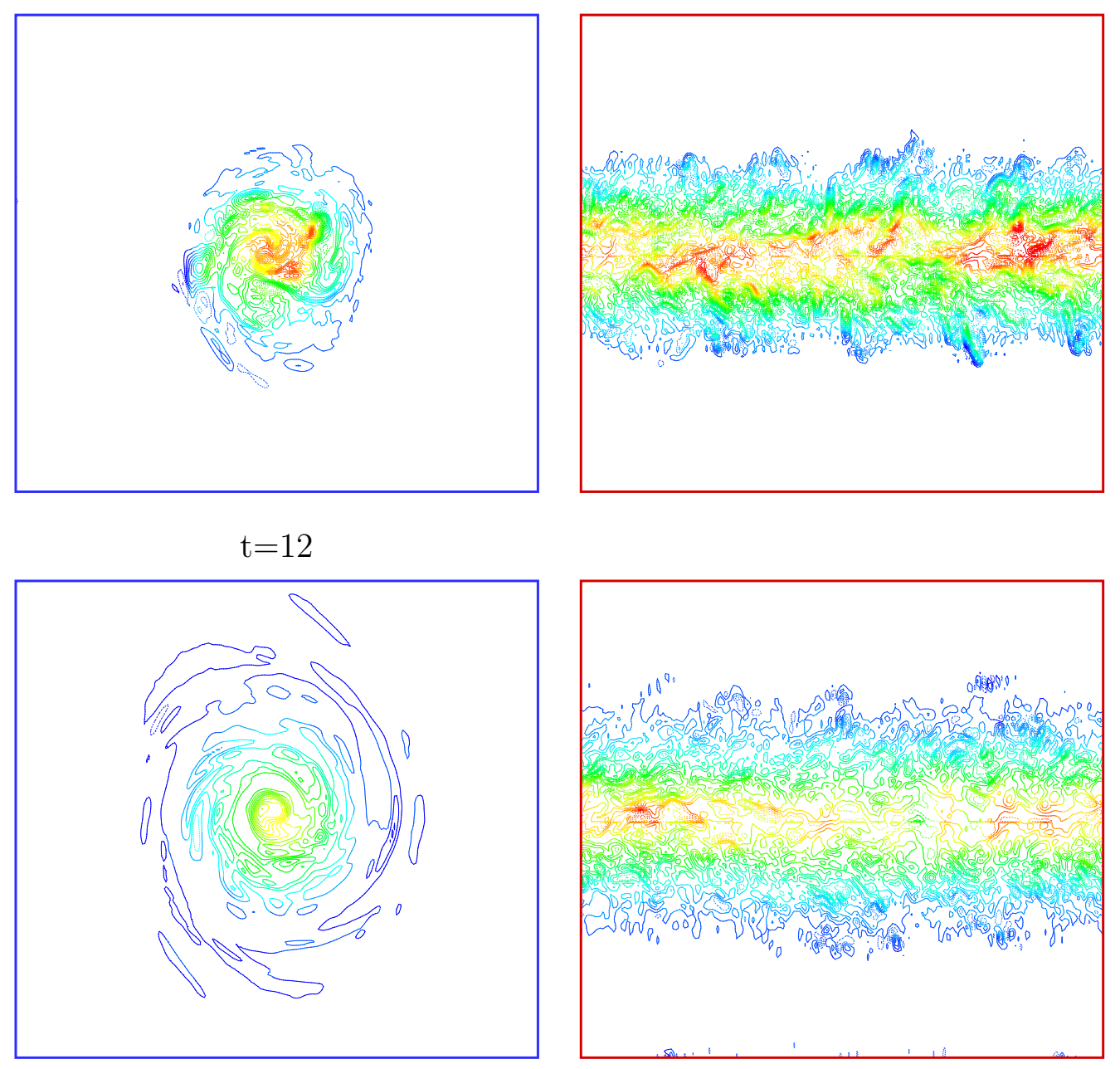

$$
t=20
$$

Figure 7.5: Contours of axial vorticity (left) and azimuthal vorticity (right) colored by axial velocity at time 12 and 20 on slices through one vortex as shown in the top figure. $R e=\Gamma / 2 \pi \nu=3000$. 


\subsubsection{Cooperative instabilities}

At slightly later times, Figure 7.2(d) shows that small amplitude kinks begin to become apparent in both the $q_{0}=\infty$ and $q_{0}=1$ cases. These are a result of the more slowly growing higher-order Kelvin modes predicted by linear stability. As these modes grow, the periodic analogue of the long-wavelength Crow instability appears, bending the vortices such that sections move alternately toward and away from each neighbor. By Figure 7.2(e), which corresponds to $t=40$, a striking difference between the two flows has become apparent. In the absence of axial velocity, the WM has distorted each vortex to the extent that separate tubular structures can be seen surrounding a highly dissipative core region. The next snapshot captured in Figure 7.2(f) shows that analogously to the vortex pair experiments of Leweke et al ${ }^{40}$ (LW henceforth), the fluid in these structures is drawn closer to the neighboring vortices where it is quickly wrapped around by the mean circulation; the effect being magnified in regions where the CM pulls pairs of vortices closer together, increasing the local strain rate and hence the WM growth rate. Ultimately this exchange of fluid between counter-rotating pairs results in a rapid decrease in circulation and explosive growth in dissipation. In contrast, the helical structure that results from the axial velocity is observed to persist and while the WM is still present, the flow appears to be more resistant to the aforementioned phenomenon. This is seen from the volume averaged quantities in Figure 7.1 where the onset of both the sharp decrease in circulation and growth in dissipation is delayed.

At later times the dissipation peaks in both cases before undergoing a rapid reduction in accordance with the significant cancellation of opposite signed vorticity. This maxima is significantly higher for the $q_{0}=\infty$ case, providing further evidence of the increased mixing that occurs in the absence of axial flow. As pointed out by LW, the final state is different from the organized array of vortex rings that would result from the reconnection of the primary structures if only the CM were present. The presence of the WM increases mixing and hence the redistribution of energy from the large scale structures to the small scales where it is dissipated. In the case of the vortex 
pair, Figure 23(d) of LW shows that the flow evolves to a state of periodic regions of small scale motion separated by thin vortical structures. The authors suggest that this is a result of a pressure gradient between regions where the primary vortex is intact and where it has broken up due to the $\mathrm{CM}$ bringing the pair closer together resulting in the pressure relaxing towards the ambient as discussed by Saffman ${ }^{60}$; the axial velocity that results transports fluid away from the latter region leaving "skeletons of the initial vortex pair". For the periodic case one would expect similar behavior, over a shorter wavelength however as the CM pulls each vortex alternately closer to both neighbors. Plots of passive scalar concentration in Figures 7.6 and 7.7 show this phenomenon beginning to occur where regions of higher concentration can be seen at the points of zero amplitude displacement of the CM. Visual comparison with the experiments at later times was not possible as the high Schmidt number of dye could not be simulated due to computational resolution constraints; at $S c=1$, higher diffusion makes visualization difficult.

\subsubsection{Circulation}

LW compute the circulation evolution around sections of one vortex in planes where the pairs are brought closer together and where they are separated. The important time scale for this phenomenon is the reciprocal of the strain rate felt at the center of each vortex due to all neighbors since this determines the growth rate of the WM instabilities. As discussed in Section 5.1.3 this strain rate takes the value given by (5.9) and (5.10) for the case of the vortex pair, and the M\&M vortex array respectively.

Figure 7.8 shows $\bar{\Gamma}$ for $q_{0}=\infty$ with the experimental points of LW compared to our results, each plotted against $\gamma t$ and with abscissa shifted such that the onset of the circulation decay occurs at about the same time. Good agreement is observed for the initial period of decay where the rate is approximately the same in both experimental planes. At later times, the circulation flattens off when the skeleton vortices appear where the $\mathrm{CM}$ brings the pair closer together, and continues to decrease in regions identified as consisting of small scale motion. The decay rate of $\bar{\Gamma}$ decreases at this 


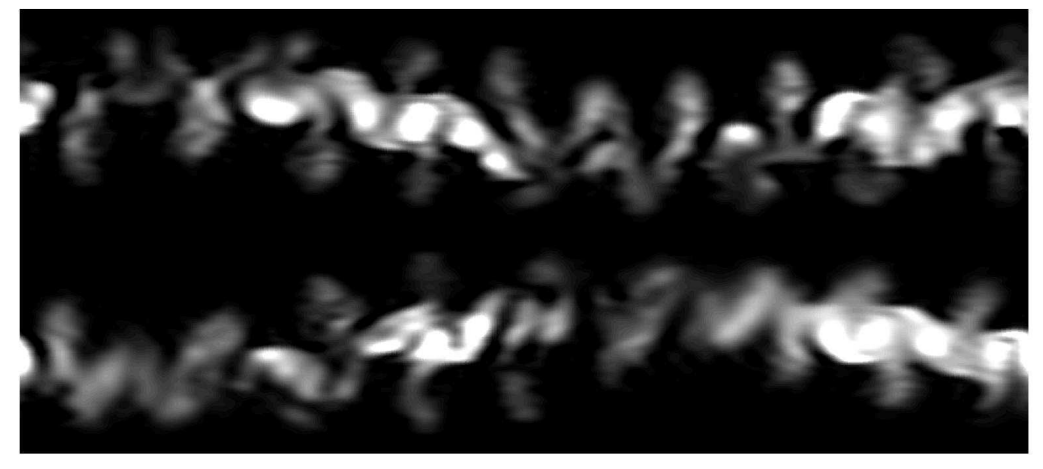

(a)

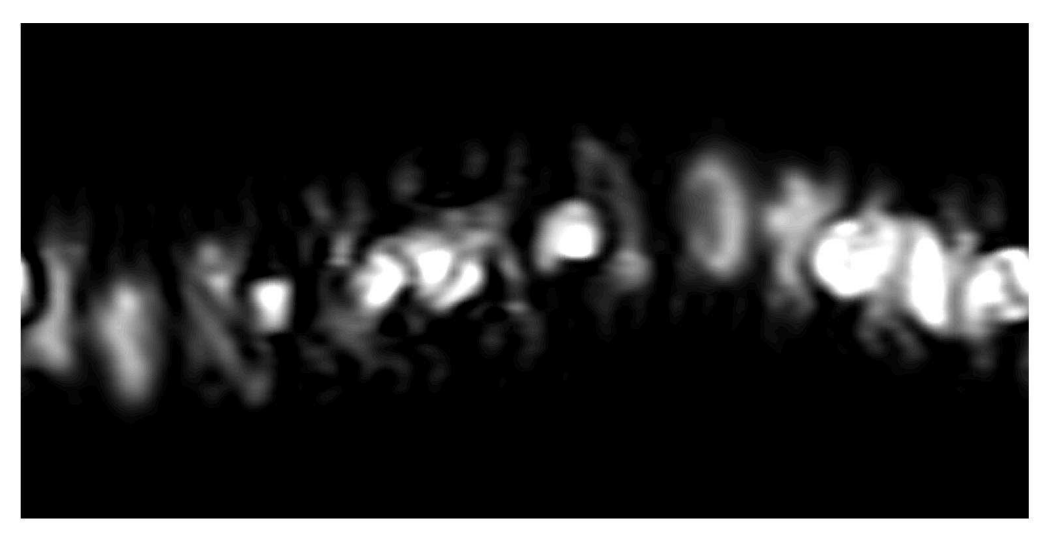

(b)

Figure 7.6: Contours of passive scalar for the $R e=\Gamma / 2 \pi \nu=1000, q_{0}=\infty \mathrm{M} \& \mathrm{M}$ vortex at time 45 on slices through the core of the vortex in the xy plane (a) and the yz plane (b). 


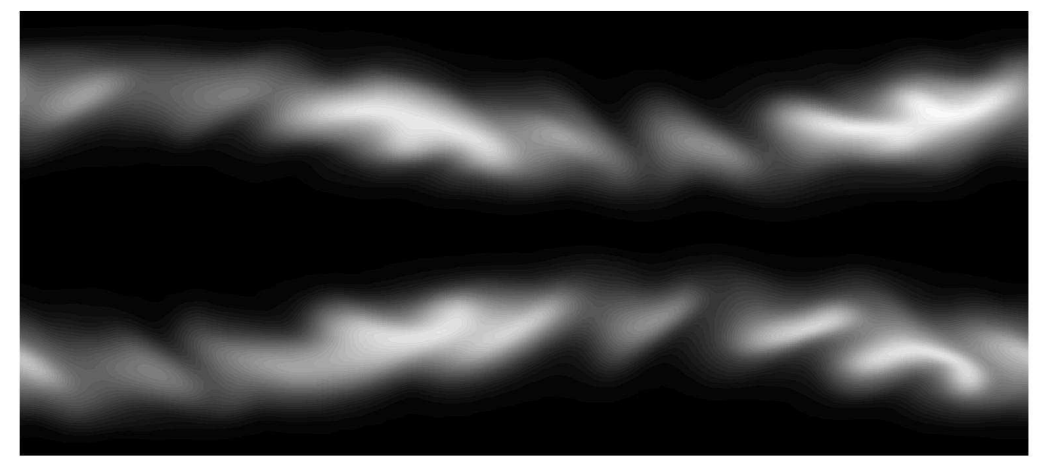

(a)

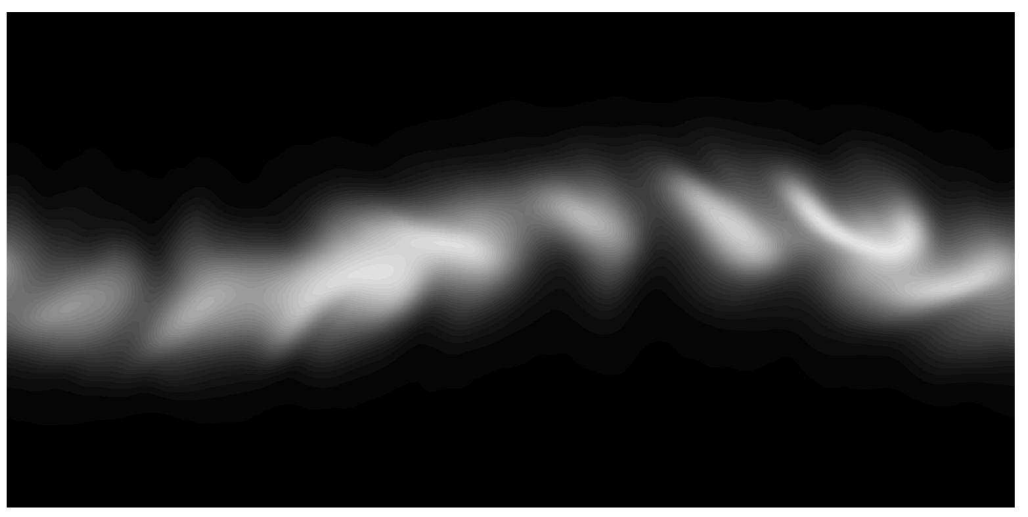

(b)

Figure 7.7: Contours of passive scalar for the $R e=\Gamma / 2 \pi \nu=1000, q_{0}=1 \mathrm{M} \& \mathrm{M}$ vortex at time 45 on slices through the core of the vortex in the xy plane (a) and the yz plane (b). 


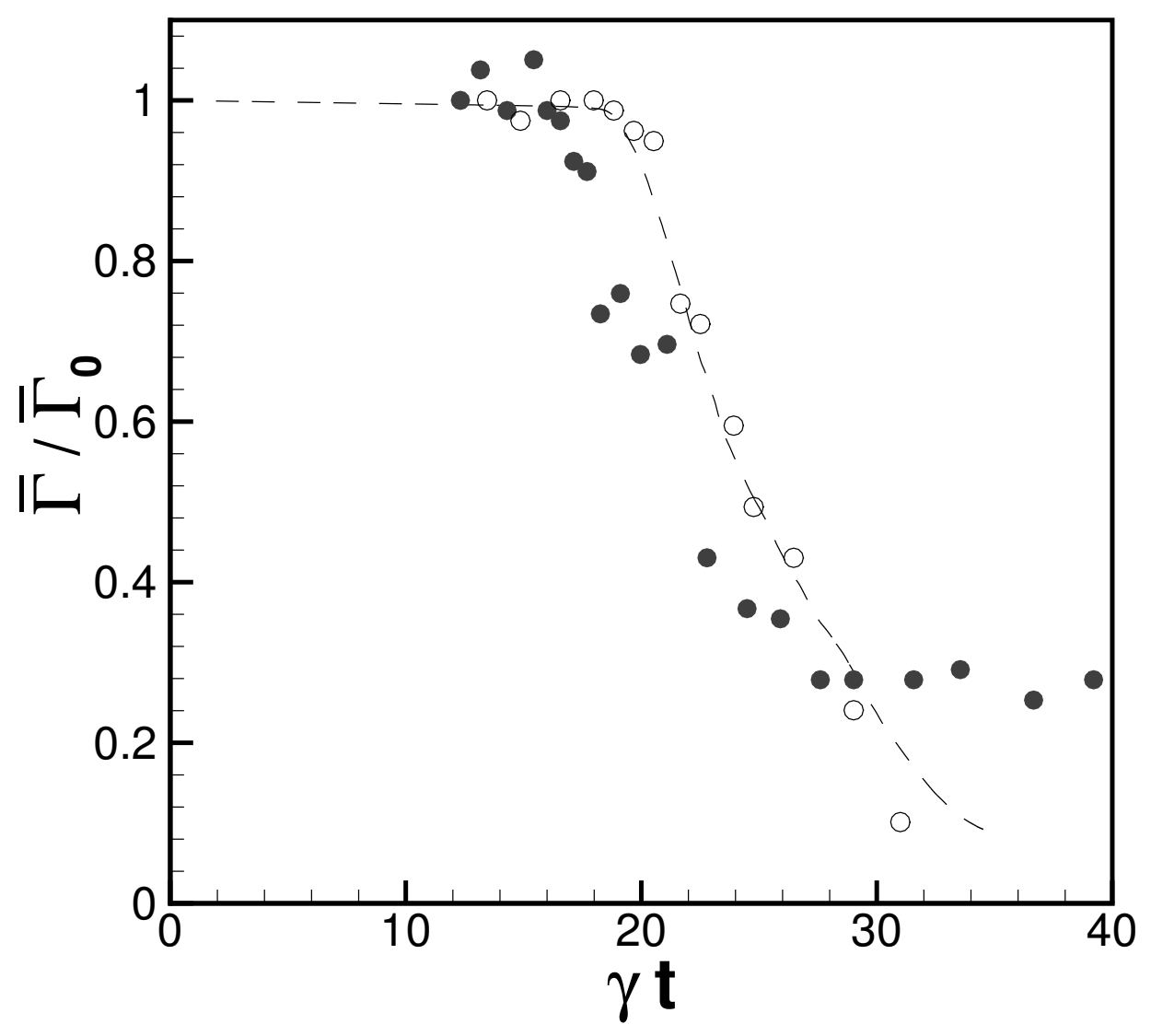

Figure 7.8: Circulation comparison with the experiments of Leweke et al ${ }^{40}$ (Figure 26), with abscissa rescaled to the present non-dimensionalization and shifted to make the onset of decay coincide. Solid circles represent data in a plane where the vortices are brought closer together and open symbols where they are separated. The dashed line is the averaged circulation for the $R e=\Gamma / 2 \pi \nu=1000, q_{0}=\infty$ case. 
time which is consistent when viewed as an average of these two phenomena. By this late time, the enstrophy level has returned almost to initial levels and the energy has decreased by a factor of five.

\subsubsection{Alignment Statistics}

We now consider the statistics of alignment between the eigenvectors of $S_{i j}$ and $\omega_{i}$, the former being denoted by $\left(\hat{e}_{i}^{1}, \hat{e}_{i}^{2}, \hat{e}_{i}^{3}\right)$ ordered according to the corresponding eigenvalues $\left(\lambda_{1}, \lambda_{2}, \lambda_{3}\right)$, with $\lambda_{3}>\lambda_{2}>\lambda_{1}$ and $\lambda_{1}+\lambda_{2}+\lambda_{3}=0$. We determine the probability density function (PDF) of alignment between a vector and a symmetric tensor in a three-dimensional coordinate system ${ }^{71}$. Local coordinates are used, defined by the orthogonal eigenvectors of $S_{i j}, \omega_{i}$ being described through its polar angle $\theta$ and azimuthal angle $\phi$ relative to $\hat{e}_{i}^{3}$ and $\hat{e}_{i}^{2}$ respectively. There is no distinction between angles in the range $(0, \pi / 2)$ and $(\pi / 2, \pi)$. Joint PDF's of $\phi$ and $\cos (\theta)$ are computed. Finally, since the present study is conducted on a domain of infinite physical extent, on which the volume coverage of turbulent flow is zero, the PDF's are presented thresholded on dissipation exceeding $10 \%$ of its maximum.

Results obtained at six time instances (corresponding to those in the visualization in Figure 7.2) are shown in Figure 7.9 for the case $q_{0}=1$. While the $q_{0}=\infty$ initial condition corresponds to a delta function PDF at $\phi=0, \cos (\theta)=0$, the presence of axial velocity leads to a more widely distributed band of alignment as shown in Figure 7.9(a). As the flow develops through the previously mentioned stages, Figures 7.9(b-e) show that the preferred alignment shifts toward smaller values of $\phi$ and $\cos (\theta)$. By time $t=45$, it can be seen from Figure $7.9(\mathrm{f})$, that the flow has evolved to a state in which the vorticity vector aligns itself preferentially with the eigen direction corresponding to the intermediate eigenvalue of the rate of strain tensor. This tendency is similar to that observed in previous studies of isotropic turbulence 32,71 . 
(a)

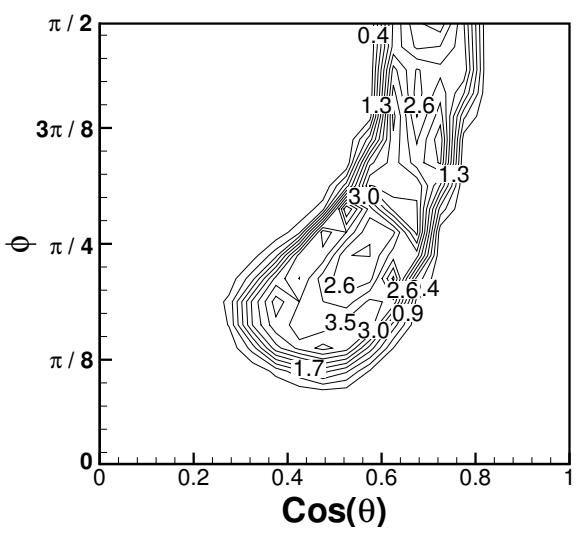

(c)

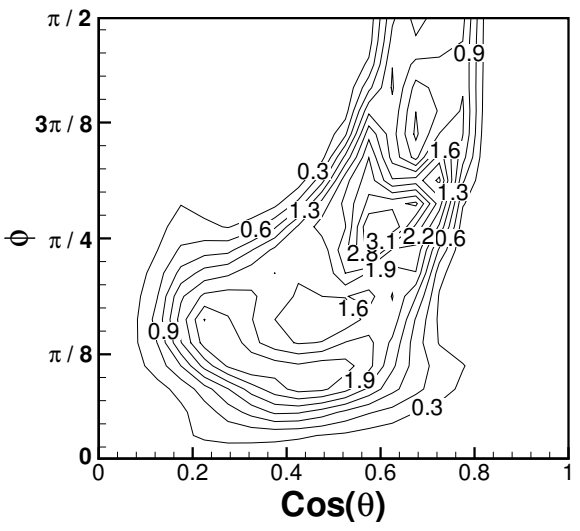

(e)

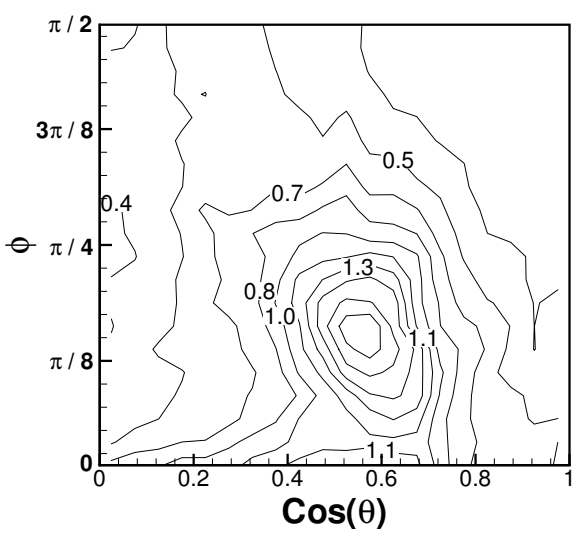

(b)
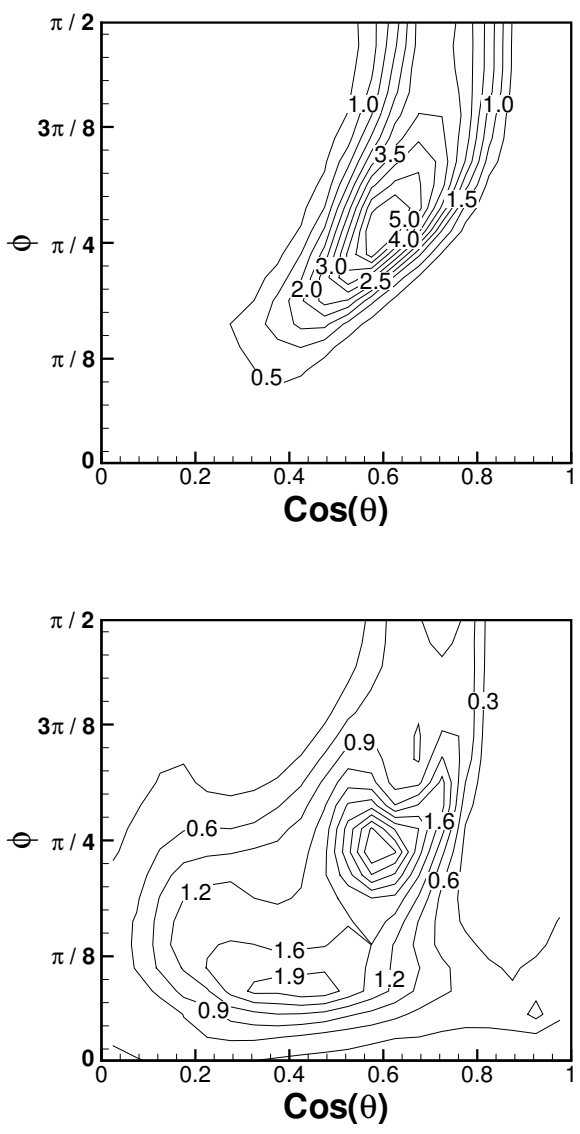

(d)

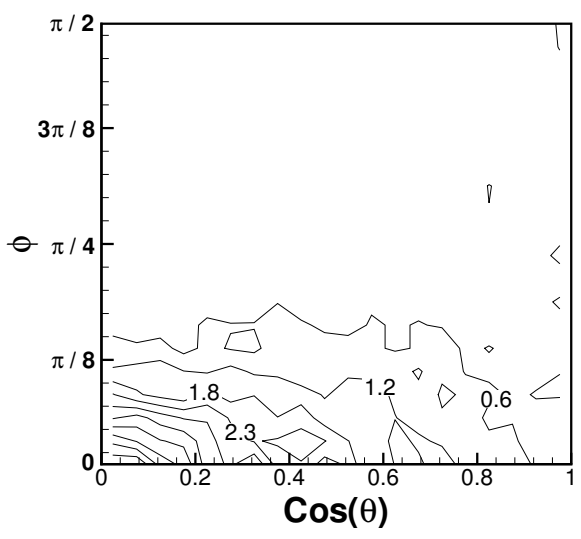

Figure 7.9: Joint PDF of the alignment between the vorticity vector and the eigenvectors of $S_{i j}$ for $q_{0}=1$ at (a) $\mathrm{t}=0$, (b) $\mathrm{t}=12$, (c) $\mathrm{t}=20$, (d) $\mathrm{t}=32$, (e) $\mathrm{t}=45$ and (f) $\mathrm{t}=50.20 \times 20$ Bins of equal widths in each direction are used to compute the PDF's. 


\subsubsection{Effect of Resolution and Domain Size}

Since the computational realization of the flow exhibits no true homogeneous directions, meaningful spectra are difficult to interpret; the domain comprises only one axial wavelength of the most unstable Crow mode and only one period of the vortex array.* Two-dimensional spectra in the $k_{1}-k_{2}$ plane at $\mathrm{z}=0$, are shown at two time instants in Figure 7.10. It can be seen that the energy falls off several orders of magnitude by the highest wave-numbers. More smooth spectra could be computed by simulating many axial wavelengths, or alternatively by performing an ensemble average over simulations with different initial perturbations. Such a computationally intensive task is beyond the scope of this work, and so a different means of testing the resolution is pursued.

We consider (7.2) which is satisfied exactly if the DNS is fully resolved. Figures 7.11 (a) \& (b) show a comparison between the terms in this equation and the resulting relative error as the simulation progresses. For most of the simulation, the error is very small; however, at later times it increases to the order of $1 \%$. This is most likely due to the $\mathrm{CM}$ pulling the vortices in the $x_{3}$ direction into regions where the grid is coarser. While this indicates that the simulation is not fully resolved at late times, the error is small, especially when one considers that dissipation takes place at the small scales. To test this, simulations were performed using twice as many modes in $x_{3}$ while maintaining the same core resolution. This has the effect of increasing the resolution at larger $x_{3}$ values and resulted in an error reduction of an order of magnitude at times when the long wavelength modes distort the vortices into these regions. For both cases, with and without axial flow, no discernible differences were seen in the observed phenomena during the time period of interest.

Another effect that must be considered is the influence of the axial domain size, since this imposes a periodicity onto the solution, restricting the spectrum of permissible instability modes. While this is a fundamental problem with the temporal

\footnotetext{
*only for an infinite number of axial wavelengths and/or periods of the array would the domain exhibit truly homogeneous direction(s).
} 
(a)

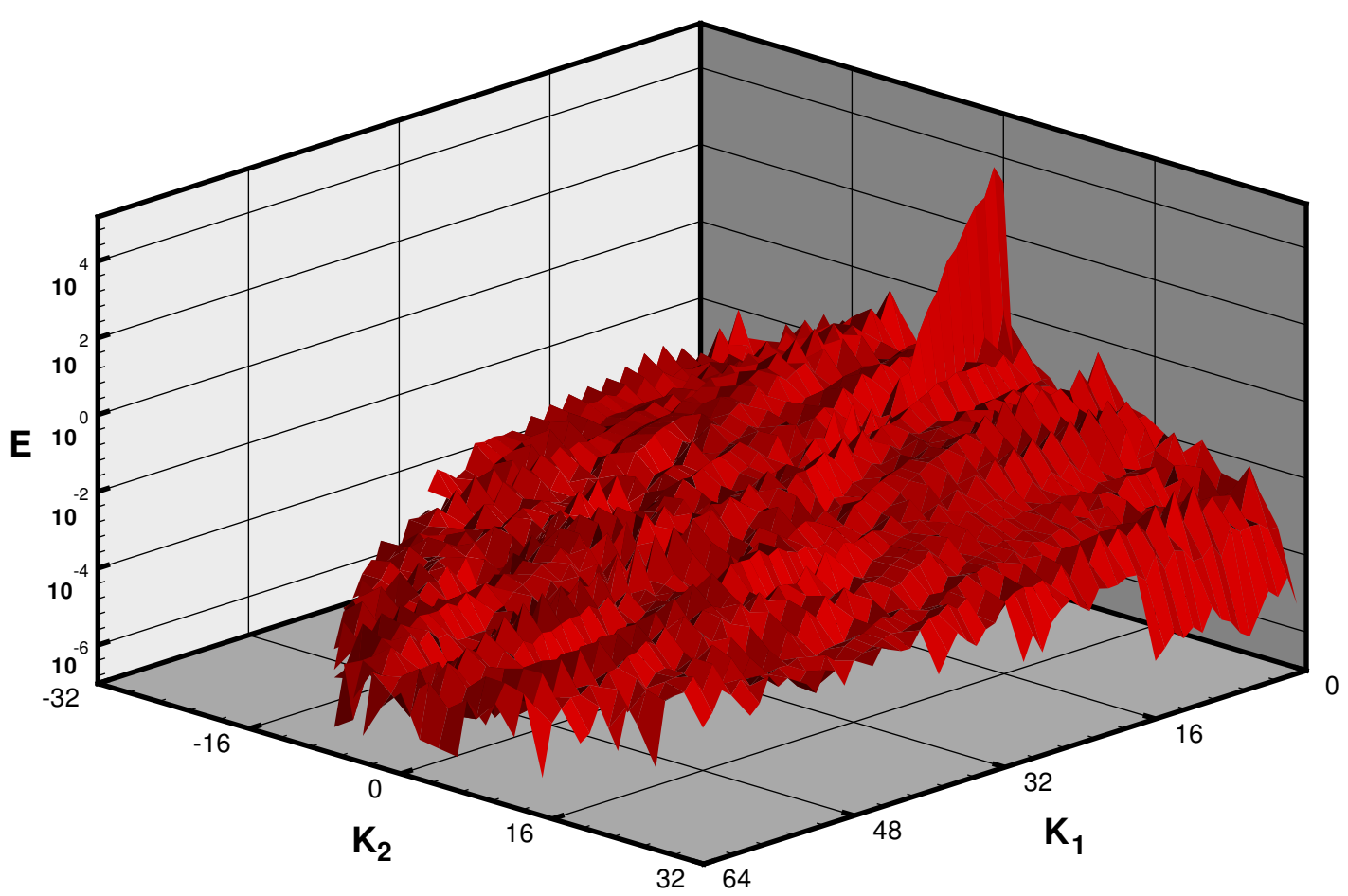

(b)

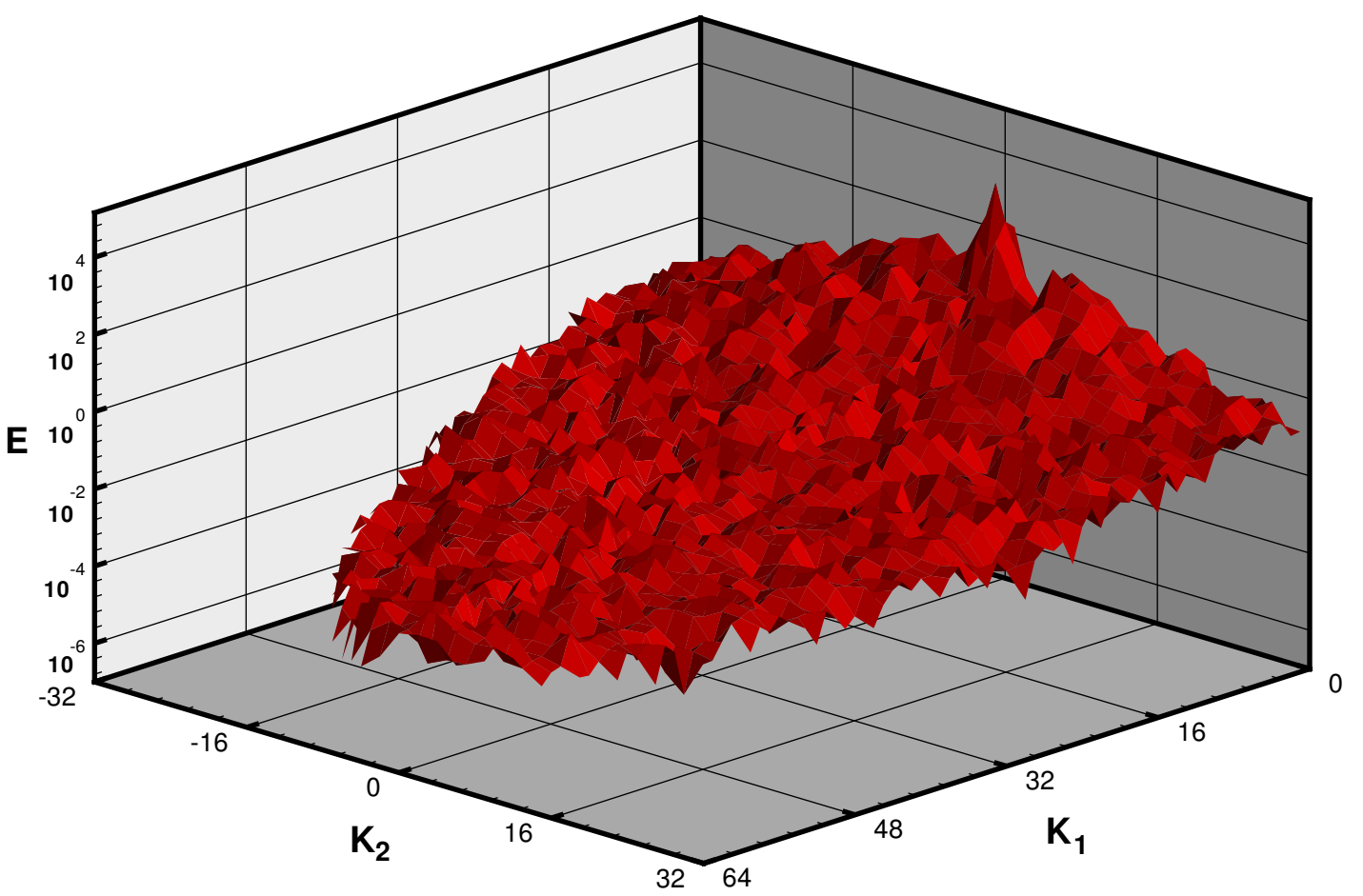

Figure 7.10: $k_{1}-k_{2}$ spectra in the plane $\mathrm{z}=0$. (a) $q_{0}=1, \mathrm{t}=12$. (b) $q_{0}=0, \mathrm{t}=45$. 
(a)
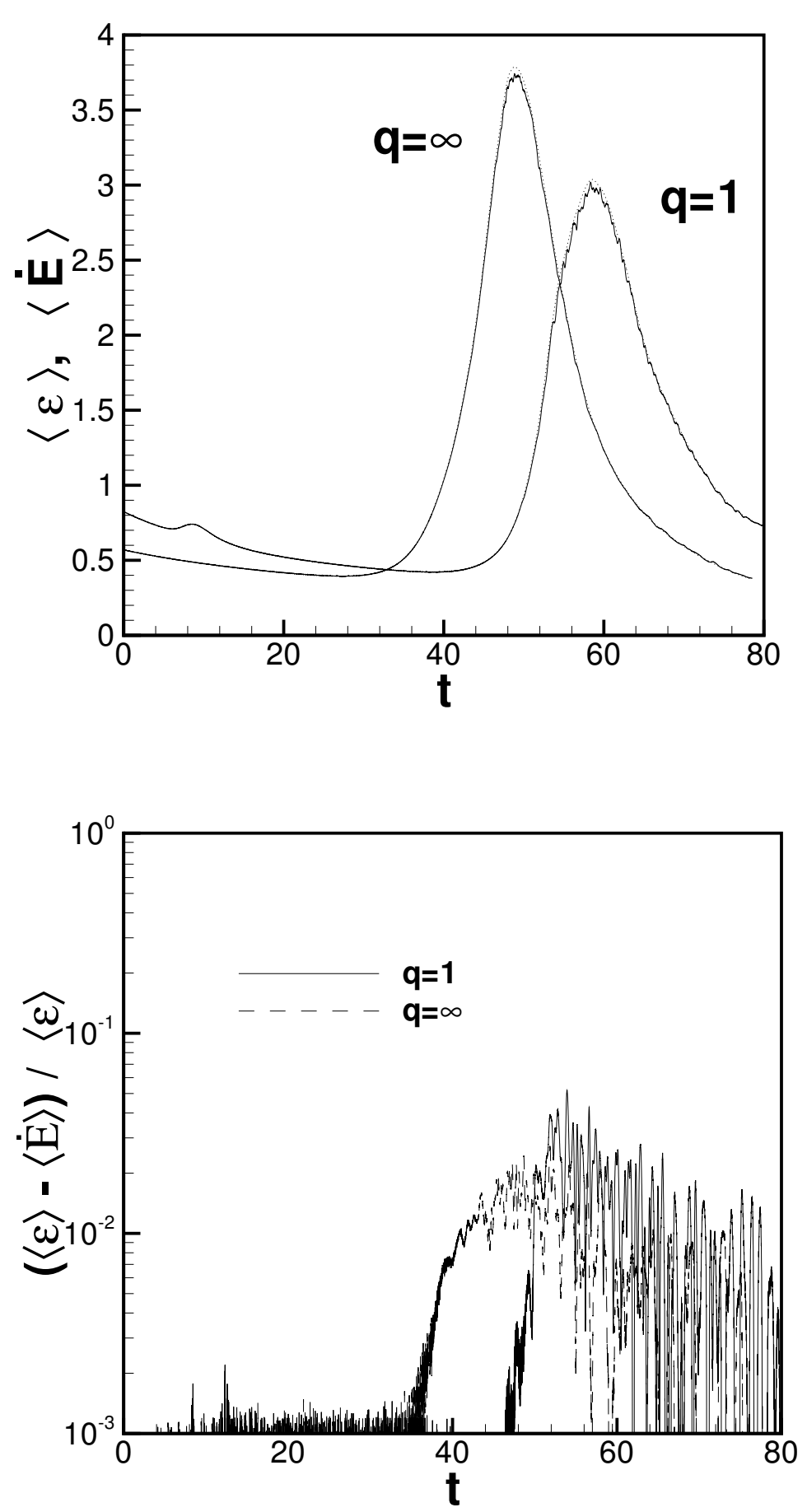

(b)

Figure 7.11: Resolution measurements for the $R e=\Gamma / 2 \pi \nu=1000 \mathrm{M} \& \mathrm{M}$ vortex with $C=0.9$ for $q_{0}=1$ and $q_{0}=\infty$ (a) rate of change of kinetic energy compared to dissipation. (b) relative error between the two. 
approach, simulations on a domain twice as long axially showed very similar results. This simulation still does not capture all the long wavelength modes; however, it is felt that the main role that this instability plays is in bringing parts of vortex pairs sufficiently close for disintegration of the vortex to initiate via the short wavelength modes. 


\section{Chapter 8}

\section{Large-Eddy Simulation}

The physical space formulation of the Stretched-Vortex Subgrid Scale model ${ }^{47}$ discussed in Chapter 3 is used to enable Large-Eddy Simulation (LES) to be performed at what theoretically could be arbitrarily high Reynolds numbers. The probability density function (PDF) that governs the alignment of the subgrid structures through $e_{i}^{v}$, is chosen to distribute proportionally between the direction of the vorticity vector $e_{i}^{\omega}$ and that of the most extensional eigenvector of the rate of strain tensor $\hat{e}_{i}^{3}$.

$$
P\left(e_{i}^{v}\right)=\mu \wp\left(e_{i}^{v} \mid \hat{e}_{i}^{3}\right)+(1-\mu) \wp\left(e_{i}^{v} \mid e_{i}^{\omega}\right),
$$

where $\wp\left(e_{i}^{v} \mid e_{i}\right)$ is the delta function probability density that $e_{i}^{v}$ is aligned with vector $e_{i}$. Presently, we use the model ansatz

$$
\mu=\frac{\lambda_{3}}{\lambda_{3}+\sqrt{\omega_{i} \omega_{i}}}
$$

This alignment model allows back scatter and has performed excellently on simulations of isotropic turbulence and Channel flow ${ }^{74}$. Physically, this is motivated by a compromise between $\mu=1$, which represents correlation of the small scale vorticity with the overall intermediate principal rate of strain (this includes the strong local strain rate provided by the subgrid vorticity), and $\mu=0$ corresponding to to alignment of the subgrid vortex with $\omega$ suggested by the idea of small scales generated through the instability of larger structures. For analysis purposes, the total 
dissipation integral for LES runs is computed as

$$
<\epsilon>=<\epsilon_{\nu}>+<\epsilon_{s g s}>
$$

and interpreted as a measure of the turbulence intensity in accordance with (7.5).

\subsection{Flow Evolution Summary}

Initially, simulations were performed at $R e=20,000$ as it is thought to be the limit where the mixing transition has occurred and fully-developed turbulence is obtained

16. Once again, the axial extent of the domain is set to contain unit wavelength of the most unstable Crow mode. Each vortex in the array (1/2 domain) is resolved by $64 \times 64 \times 256$, however, the initial core is still described by 24 points in the diameter, the extra resolution in the infinite direction included to reduce truncation errors associated with the grid stretching function; since the subgrid contains energy now.

Figure 8.1 shows a comparison of the evolution of $\langle\epsilon\rangle$ and Figure 8.2, energy and $\bar{\Gamma}$, for $q=\infty$ and $q=1$ Flow visualization through isosurfaces of $Q=5$ shaded with dissipation are shown at several snapshots in Figure 8.3. As in the DNS, the presence of axial flow initially causes each vortex to develop helical instability modes leading to increased levels of turbulence. This time, however, the increase in dissipation is greater and despite the weakening of the instability mechanism, it fails to drop to its original level before the cooperative instabilities take over. As observed in the DNS, the effect of axial velocity is to prolong the onset of vortex breakdown, but at larger $R e$ the delay is longer, presumably due to the greater intensity of the helical structure within each vortex. 


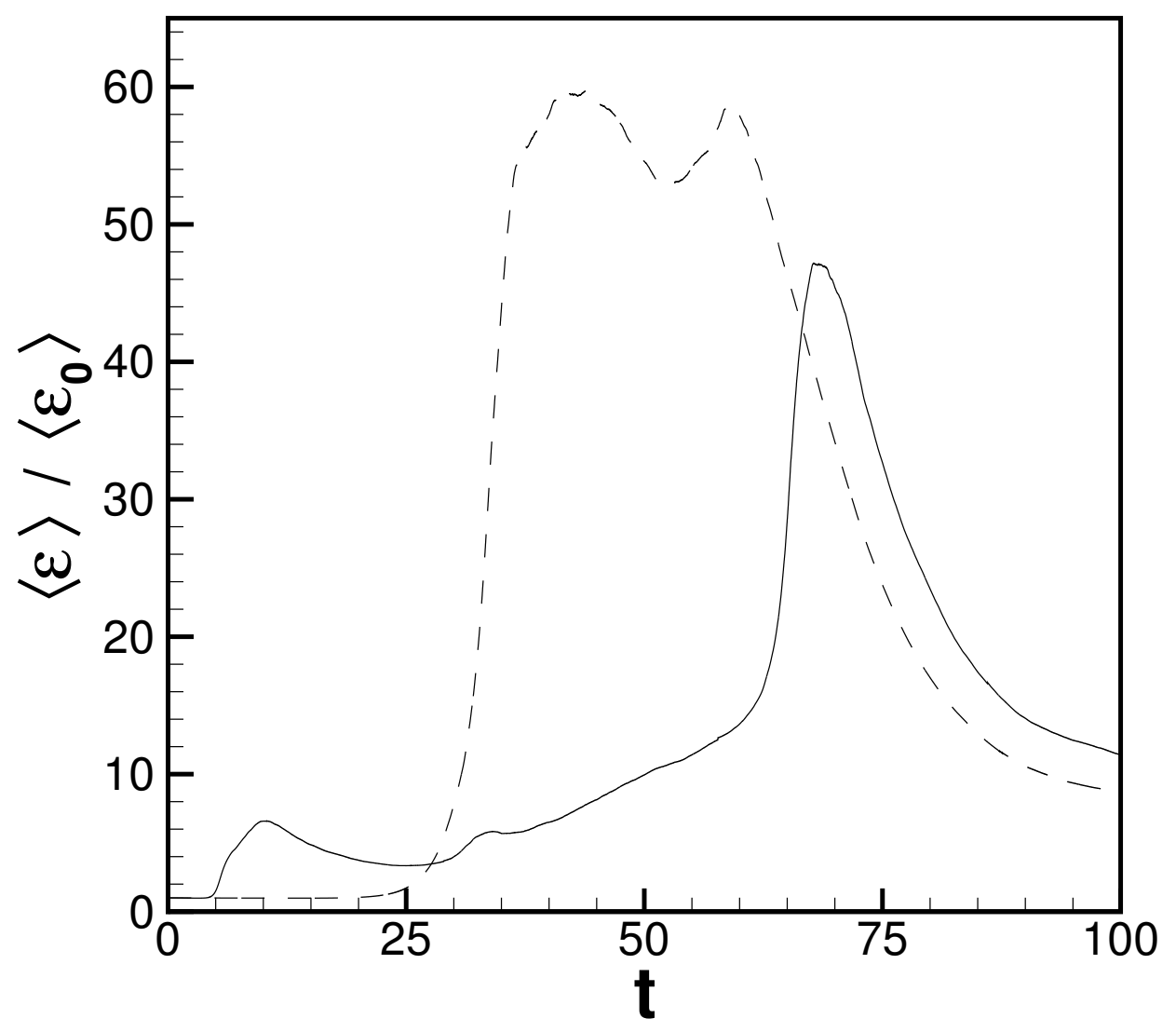

Figure 8.1: Evolution of dissipation for $R e=\Gamma / 2 \pi \nu=20,000$ with $q_{0}=1$ (solid lines) and $q_{0}=\infty$ (dashed lines). 


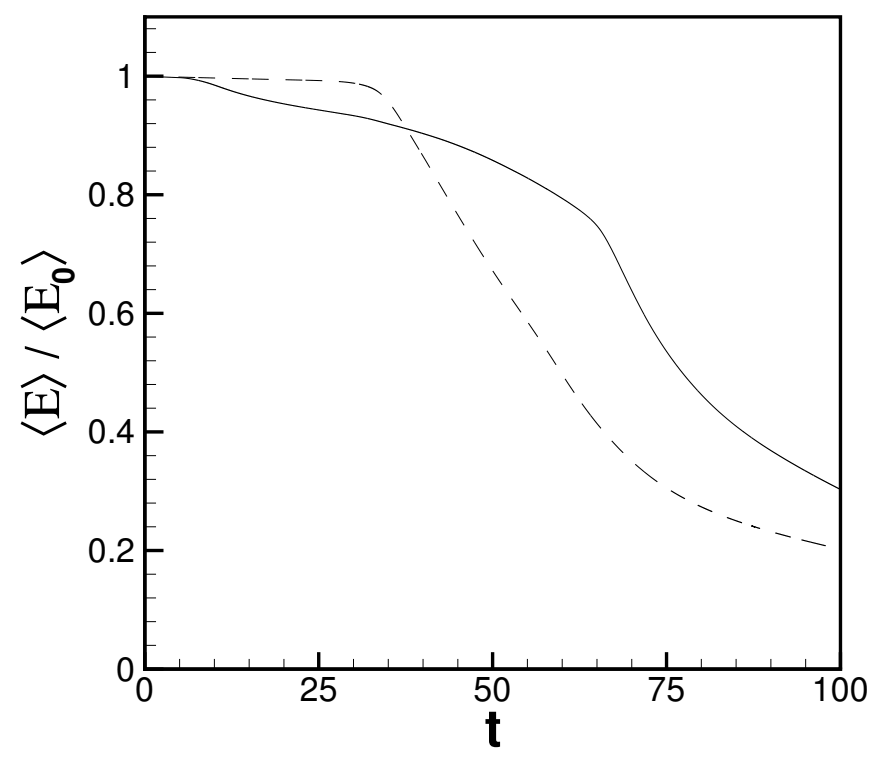

(a)

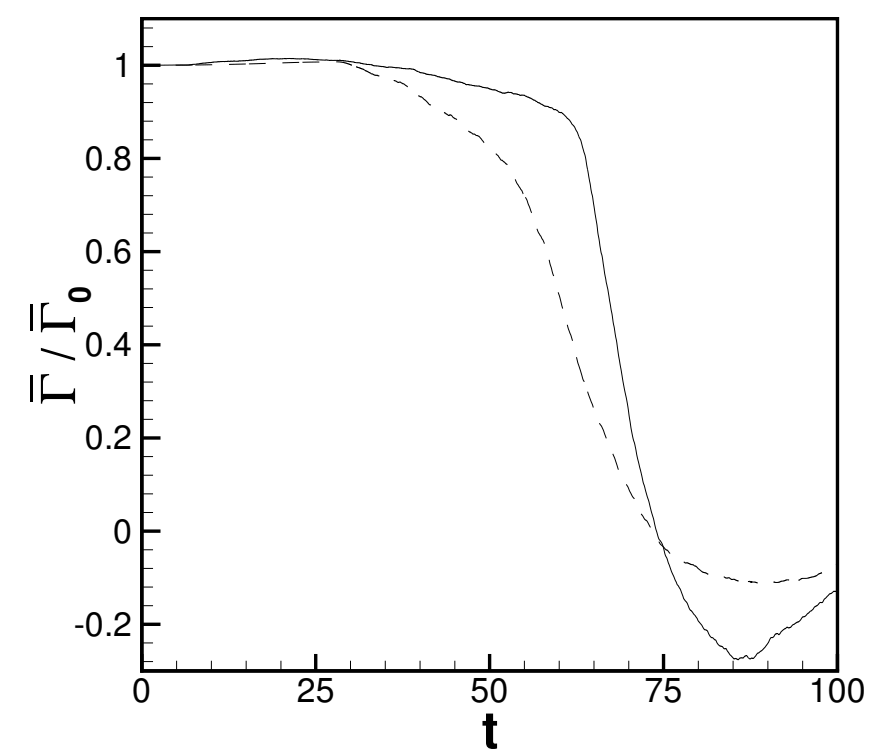

(b)

Figure 8.2: Evolution of (a) energy and (b) circulation for $R e=\Gamma / 2 \pi \nu=20,000$ with $q_{0}=1$ (solid lines) and $q_{0}=\infty$ (dashed lines). 

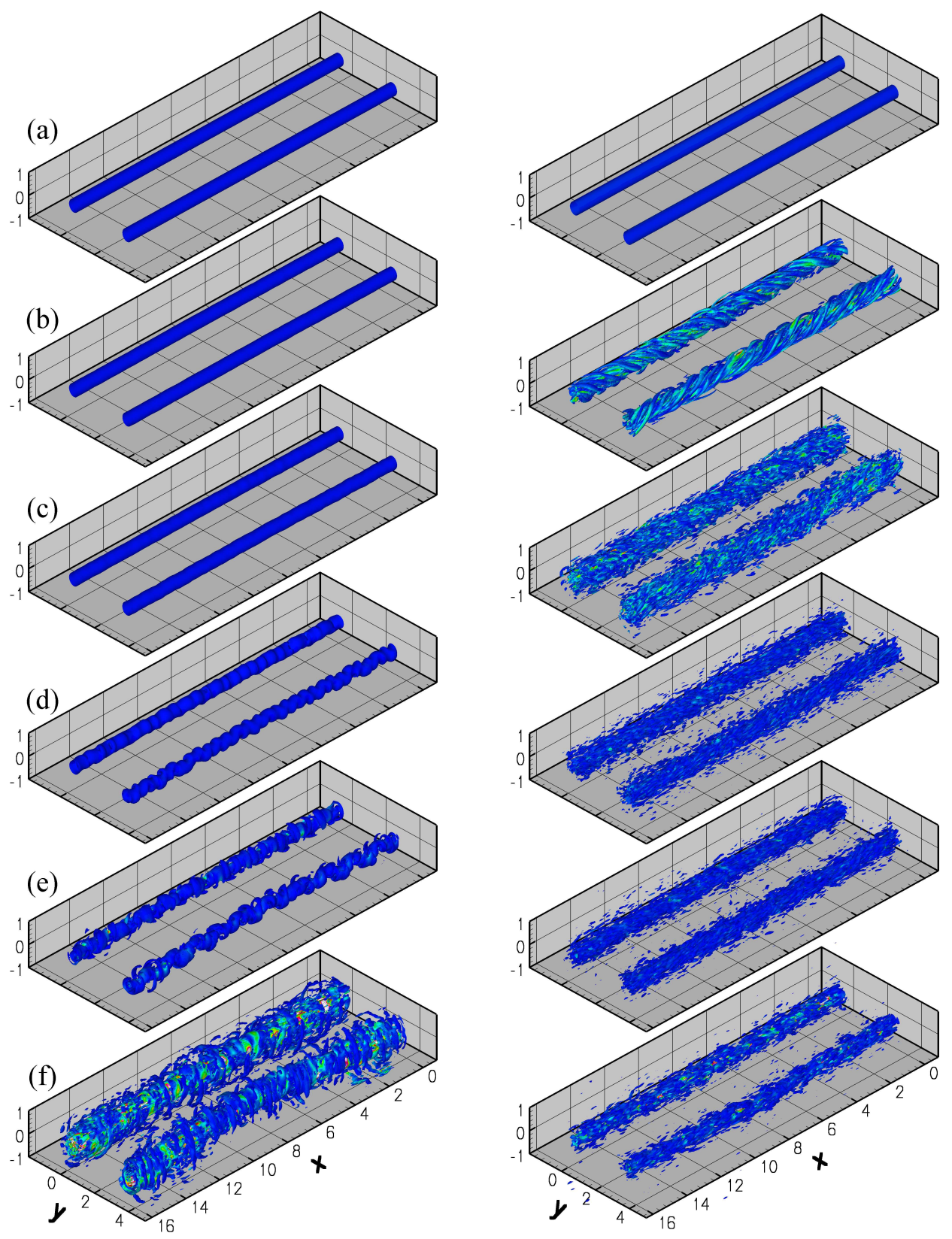

Figure 8.3: Flow visualization comparison through an isosurface with $Q=5$ on which contours of dissipation are plotted for the $R e=\Gamma / 2 \pi \nu=20,000$ LES of the $\mathrm{C}=0.9 \mathrm{M} \& \mathrm{M}$ vortex with $q_{0}=\infty$ left and $q_{0}=1$ right. Evolution is from top to bottom and shown at non-dimensional times (a) 0 (b) 6 (c) 11 (d) 20 (e) 26 (f) 32. 


\subsection{SGS model performance}

The performance of the subgrid model is shown in Figure 8.4 through the dissipation ratio $\epsilon_{\text {ratio }}$ defined as the proportion of the total dissipation that comes from the subgrid

$$
\epsilon_{\text {ratio }}=\frac{\left\langle\epsilon_{\text {sgs }}\right\rangle}{\left\langle\epsilon_{\nu}\right\rangle+\left\langle\epsilon_{\text {sgs }}\right\rangle} .
$$

It can be seen that, as expected, the model is most active during the WM enhanced mixing stage where the circulation decay occurs and in the case of axial velocity, the period of initial helical growth. At its peak, the model contributes approximately

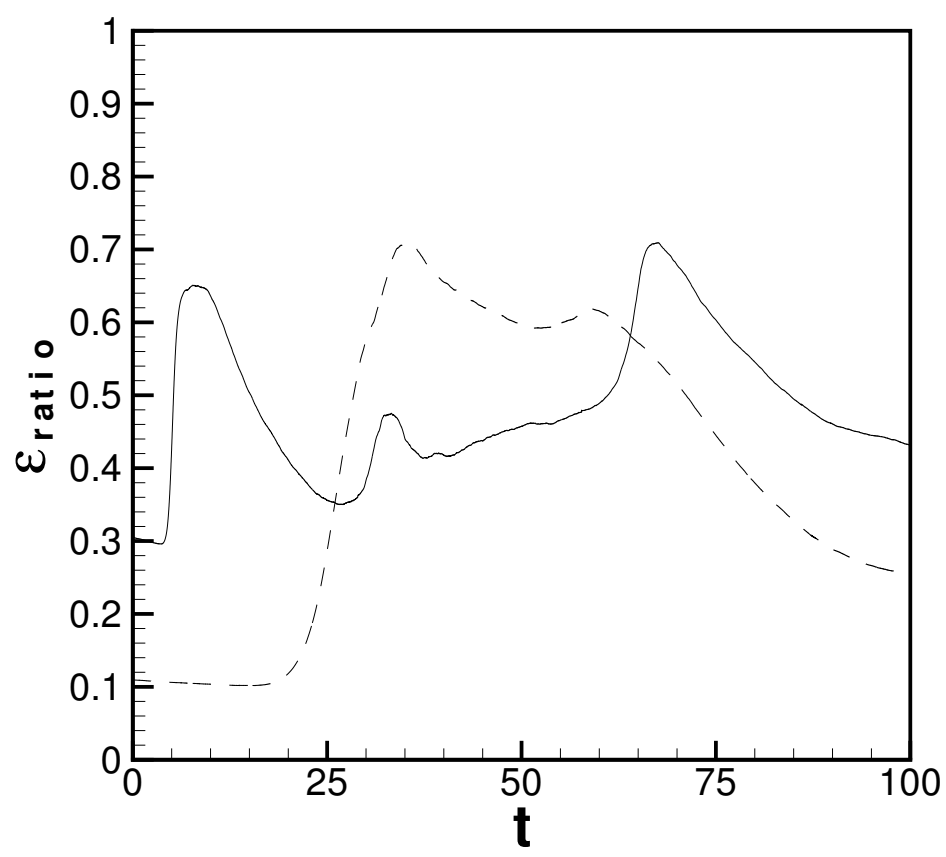

Figure 8.4: Evolution of $\epsilon_{\text {ratio }}$ for $R e=\Gamma / 2 \pi \nu=20,000$ with $q_{0}=1$ (solid lines) and $q_{0}=\infty$ (dashed lines).

$70 \%$ of the total dissipation in both cases. It is interesting to note that this is roughly the same as for the channel flow simulations of Voelkl et al. ${ }^{74}$ at $R e_{\tau}=1017$ which corresponds to a large scale Reynolds number (based on center line velocity) of around 20,000. While the macroscopic details of the two flows are quite different, 
the small scale details appear to be similar. Toward the end of the simulation, when the circulation decay is complete, the subgrid contribution diminishes indicating that the flow is becoming more resolved.

To gain further insight into the performance of the model, we consider the alignment statistics between the eigenvectors of $S_{i j}$ and the eigenvectors of the subgrid stress tensor. Tao et al. ${ }^{71}$ computed such alignment PDF's from their experiments of fully developed turbulent flow in a duct, by filtering their data to obtain resolved and subgrid scales. While it is unreasonable to expect the present subgrid model to reproduce these PDF's exactly, it is pleasing to note that the average angle between the most extensive stress and the most extensional strain rate is $\approx 39^{\circ}$, which is close to the preferred alignment angle of $34^{\circ}$ found by the experimental study.

\subsection{Early development}

Figure 8.5 shows, for $q=1$, the evolution of $\langle\epsilon\rangle /\left\langle\epsilon_{0}\right\rangle$ during this period for Reynolds numbers in the range 1000 to 20,000 . It can be seen that as $R e$ is increased so too does both the dissipation growth rate, and the peak dissipation level obtained. After this maximum, the dissipation decreases at a rate that is approximately independent of Reynolds number. At later times, the cooperative instabilities dominate as seen in the DNS; the CM pulls vortex pairs together where enhanced transport via the short-wavelength instabilities leads to the eventual disintegration of the vortex. The linear analysis suggests that the long wavelength modes and hence the timescale for initiation of this phenomena are independent of Reynolds number. We conclude that complete relaminarization of the vortex is not observed at the higher Reynolds number, as in the isolated vortex simulations of Sreedhar \& Ragab ${ }^{67}$, because that insufficient time passes for the smaller viscosity to damp out the turbulent fluctuations prior to the dominance of cooperative instabilities.

As with the DNS, the early evolution of each vortex is considered as independent from every other in the array and it is thus meaningful to speak in terms of cylindrical components. Further, radial distributions are obtained by averaging over the 


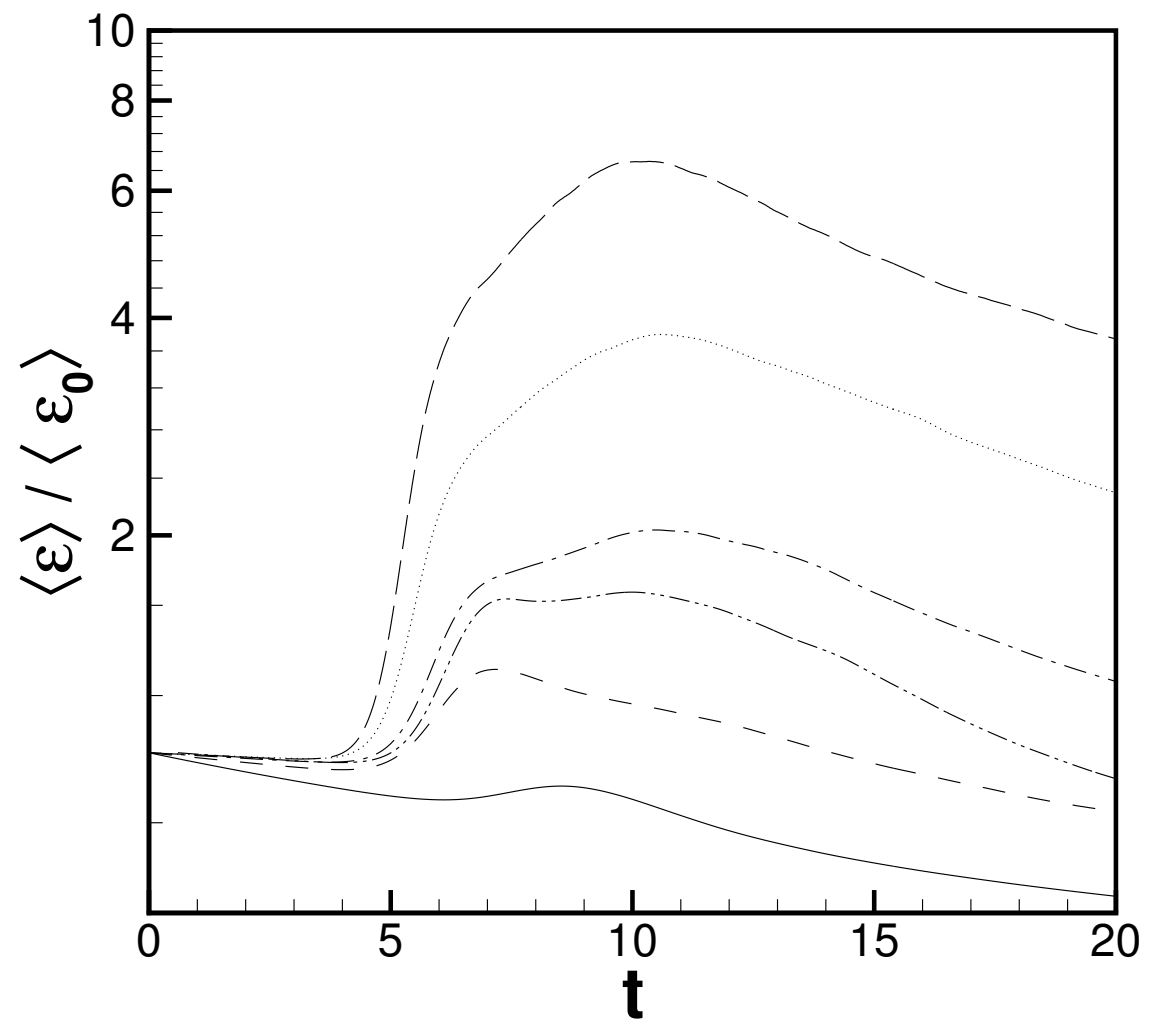

Figure 8.5: Effect of $R e$ on the early development of the vortex array with $q_{0}=1$. Dissipation is plotted at Reynolds numbers 1000, 2000, 3000, 4000, 8536, 20,000 (lower curve to upper curve). The first 3 were obtained from DNS and the latter 3 from LES.

azimuthal direction. The results are interpreted cautiously, however, since only for infinite vortex separation is this direction truly homogeneous. The distribution of circulation computed in this manner is shown in Figure 8.6. It can be seen that in accordance with the universal inner region of Hoffman and Joubert ${ }^{23}$ for turbulent vortices, a region of solid body rotation develops in the core of the vortex, followed by a transition to a region where the circulation varies logarithmically. Phillips ${ }^{50}$ considered the logarithmic region as only the limiting form for $r=r_{c}$ and formulated a similarity solution for the approximate form of the circulation as a function of radii for the turbulent trailing vortex. Good agreement is found with the current work to a radii of approximately $3 r_{c}$, beyond which the influence of the counter rotating 


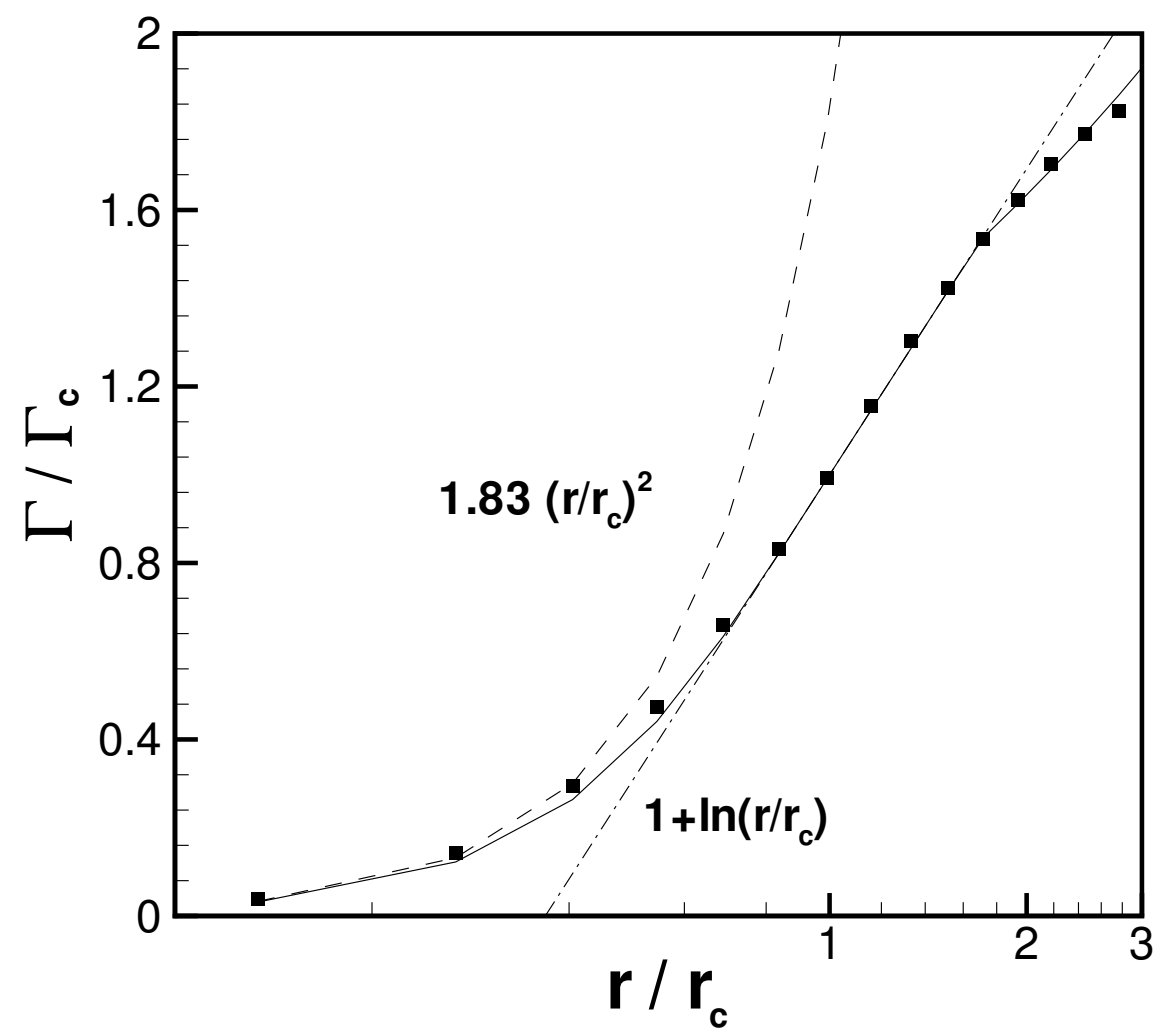

Figure 8.6: Circulation profile for $R e=\Gamma / 2 \pi \nu=20,000$ at $t=10$ (symbols) showing good agreement with the similarity solution of Phillips ${ }^{50}$ (solid line). Also shown are the inner and logarithmic regions predicted by Hoffman and Joubert ${ }^{23}$.

pair becomes substantial. Plots of the cylindrically averaged velocity and $\epsilon_{\text {ratio }}$ are shown in figure 8.7 for $R e=20,000$. As expected, the axial velocity maintains its initial bell shaped profile although its magnitude in the core decreases as it feeds turbulence generation in that region. Outside the core, however, the axial velocity remains relatively unchanged during the process. In sharp contrast to this, the model dissipation changes only slightly near the center, but becomes more active with time at larger radii, as turbulent fluctuations generated in the core propagate outwards. It is interesting that the model contribution does not die off at small radii, where the turbulent vortex is thought to be a region of laminar solid body rotation. While this appears to be true in an averaged sense, as seen from the circulation profile in 
(a)
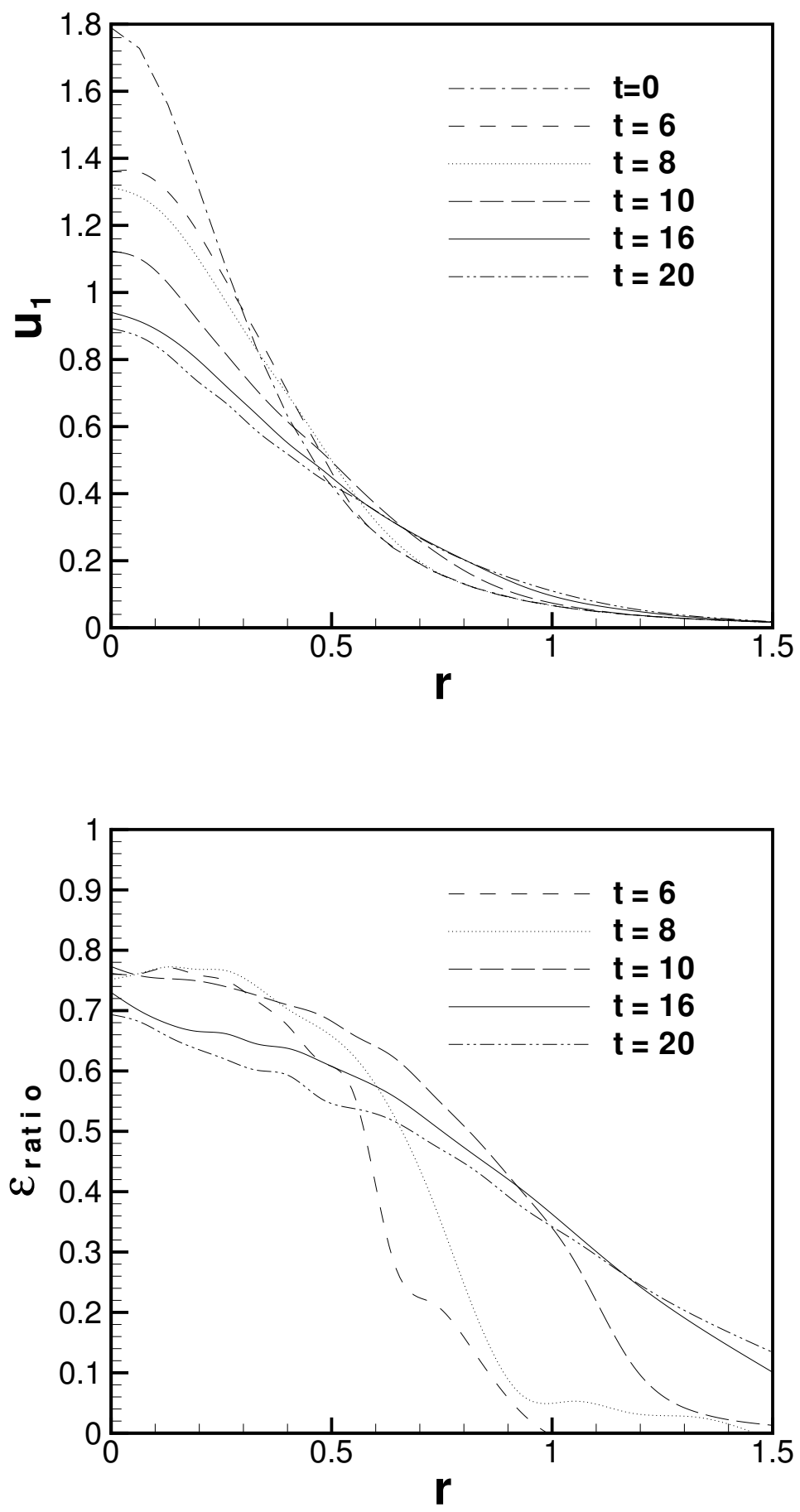

(b)

Figure 8.7: Radial distributions of (a) the $\theta$ averaged axial velocity and (b) $\epsilon_{\text {ratio }}$ at several instances in time for $q_{0}=1, R e=\Gamma / 2 \pi \nu=20,000$. 
Figure 8.6, it appears that, in agreement with previous simulations ${ }^{67}$, the inner region is characterized by high local fluctuations. This is also in keeping with linear theory, which predicts zero growth rate of small amplitude disturbances only at the very center ${ }^{25}$.

\subsection{Mixing Transition}

Konrad and Roshko ${ }^{34}\left(\mathrm{cf}\right.$. Breidenthal ${ }^{7}$ ), observed in their shear layer experiments that above a particular value of the local Reynolds number, the amount of small scale mixing is substantially increased. Dimotakis ${ }^{16}$ generalized this idea to other flows, proposing that above a critical Reynolds number, the flow transitions to a more well-mixed fully developed turbulent state. This mixing-transition takes place beyond the usual transition from laminar to unsteady flow that can often be understood in terms of hydrodynamic stability arguments. In shear layers for example it is observable as a well-identified transition in the flow; however, it is less conspicuous in other flows such as Jets which are three-dimensional even at low Reynolds numbers 16. A mixing-transition in such flows is qualitatively identifiable, however, through a Reynolds number independence of quantitative measures such as the scalar variance and viscous dissipation. Motivated by results from experiments and DNS of homogeneous turbulence in a spatially periodic cube ${ }^{68,69}$, we now consider the effect of $R e$ on $\langle\epsilon>$ as one means of identifying a mixing-transition during the early evolution of the vortex array.

Presently, velocity and length scales, $v^{*}$ and $L^{*}$, are chosen as the (time dependent) maximum azimuthal velocity component and the radial location at which it occurs. Figure 8.8 shows the dissipation non-dimensionalized in this way vs. $R e$ at several stages of the turbulent development initiated by the helical instability mechanism. The energy dissipation rate appears to be independent of viscosity at high $R e$. Further, for $R e \geq 1-2 \times 10^{4}$, it asymptotes to a value of approximately 0.1 ; somewhat higher at $t=10$. While this constant is generally recognized as a function of the flow geometry, the mixing transition concept suggests that the Reynolds number 


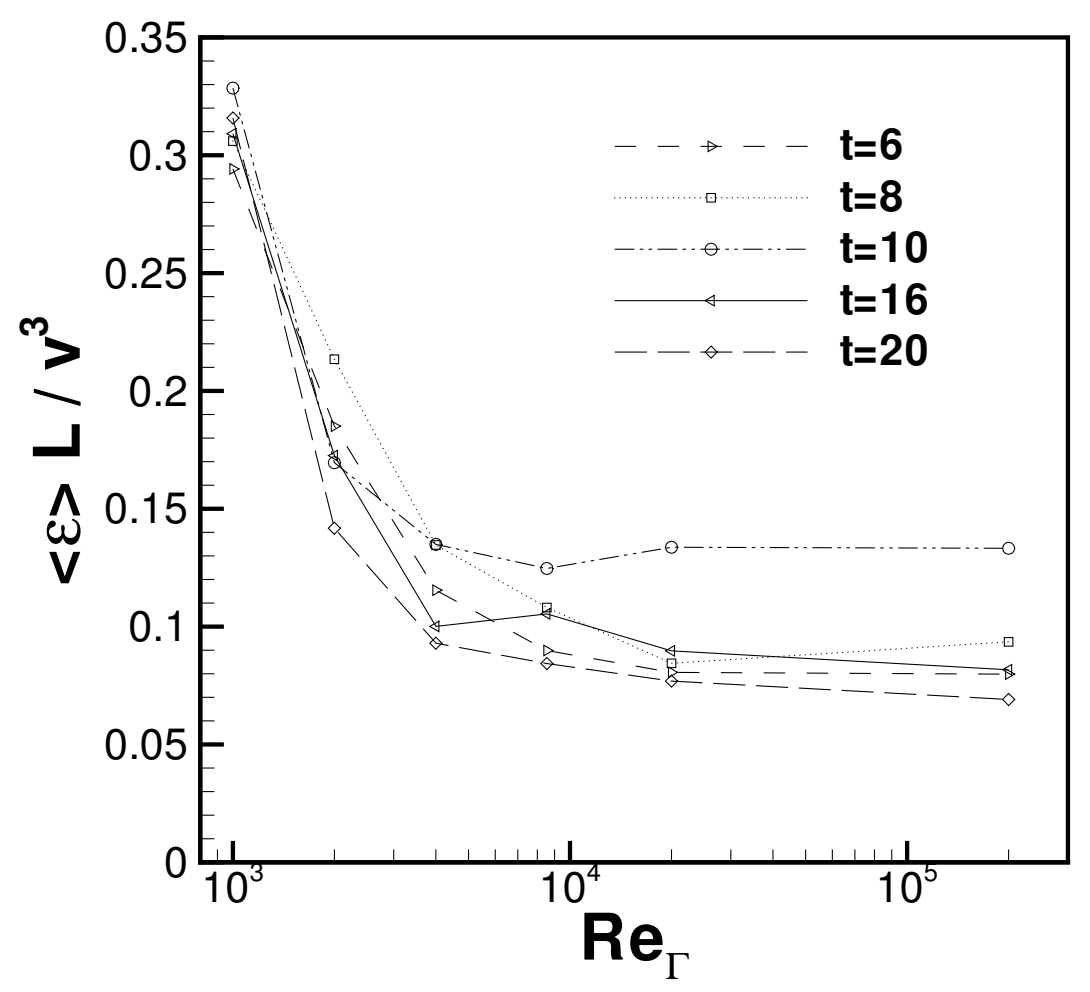

Figure 8.8: Dissipation normalized by the maximum swirl velocity and its radial location vs $R e$ at several instances in time.

above which it is reached is not. By considering experimental and numerical simulation data on a wide variety of flows, Dimotakis ${ }^{16}$ concludes that a mixing-transition occurs above a large scale Reynolds number of between $1-2 \times 10^{4}$. To support this hypothesis, the Reynolds number dependence on the vortex structure of the flow field is studied by considering isosurfaces of Q (close ups of Figures 7.2 and 8.3). Figure 8.9 (a-e) shows that while considerable difference is observed between $R e=1000$ and 20, 000, the structure remains almost unchanged when the Reynolds number is raised by an order of magnitude; two time instances have been shown for the lower viscosity cases to visualize the helical structure developing into smaller scale motions. This suggests that the mixing-transition in the present flow occurs by $R e=2 \times 10^{4}$ and more profoundly that the flow field at this value is representative of what would be obtained at all higher Reynolds numbers. 


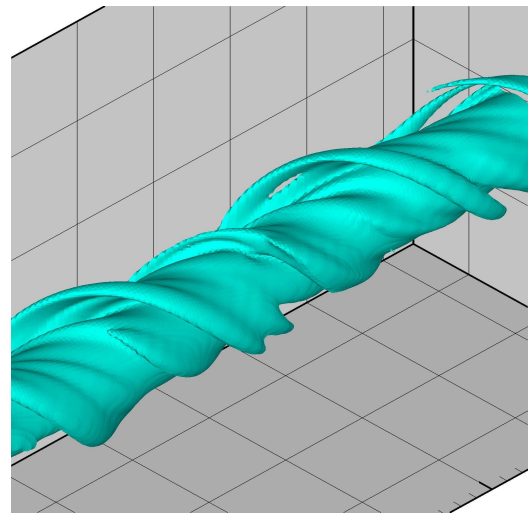

(a)

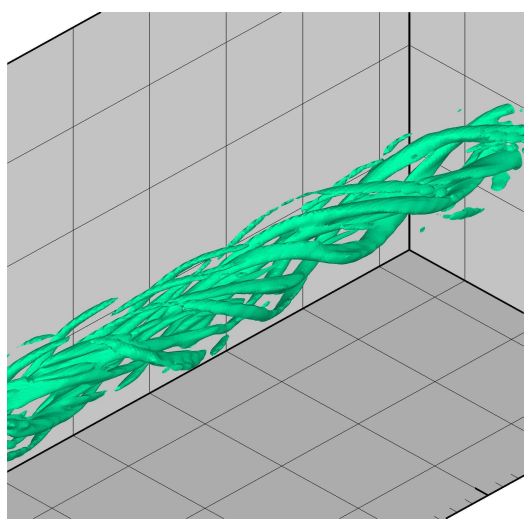

(b)

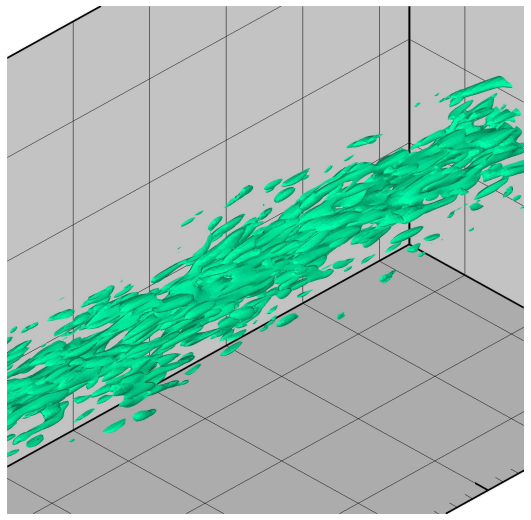

(d)

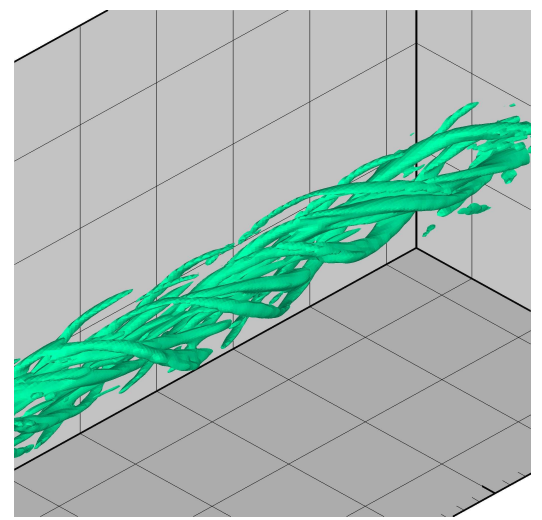

(c)

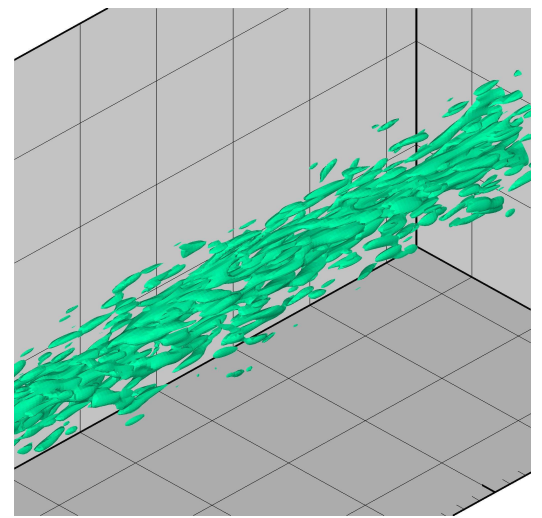

(e)

Figure 8.9: Enlarged views of a section of one vortex in the array showing the effect of $R e$ on the flow structure through an isosurface of $\mathrm{Q}$. (a) $R e=\Gamma / 2 \pi \nu=1000, Q=1, t=12$. (b) \& (c) $Q=5, t=6, R e=20,000$ and $R e=200,000$ respectively. (d) \& (e) $Q=5$, $t=10, R e=20,000$ and $R e=200,000$ respectively. 


\section{Chapter 9}

\section{Axial Pressure Gradients}

The presence of the engine structure, the fuselage and the horizontal stabilizer, as well as flap tips during the take-off phase of real aircraft create additional co- and counterrotating vortex systems that complicate the idealized picture of an airfoil wake. It is generally believed ${ }^{64}$ that all the vortices on one side of the symmetry plane merge, resulting in a pair of counter-rotating vortices; however, experimental evidence has shown that under some loading conditions several discrete vortex systems can remain many chords downstream ${ }^{15,64}$. Figure 9.1 shows an experimental study performed by de Bruin et al. ${ }^{15}$ on a Fokker airliner model in which multiple vortices can clearly be seen. The interaction between such co-rotating systems has been the focus of much work, although the vortices are typically treated as inherently two-dimensional. Bertenyi ${ }^{4}$ studied experimentally the three-dimensional interactions that occur during co-rotating vortex merger and found that merger occurred sooner than was predicted for the corresponding two-dimensional case. It is with this motivation that we consider in greater detail the processes that occur during the formation of the trailing vortex pair.

In the previous chapters, the effect of axial velocity within the core of each vortex was shown to play an important role and, if strong enough, could cause instabilities that grow on a timescale much shorter than that for the co-operative instabilities that eventually bring opposite signed pairs together. In the absence of viscosity, a jet-like profile (away from the wing) results from the pressure in the cores of the rolled 
up vortex being lower than the ambient pressure ahead of the plane; referred to as the Bernoulli effect ${ }^{64}$. Moore and Saffman ${ }^{48}$ showed analytically that the presence of

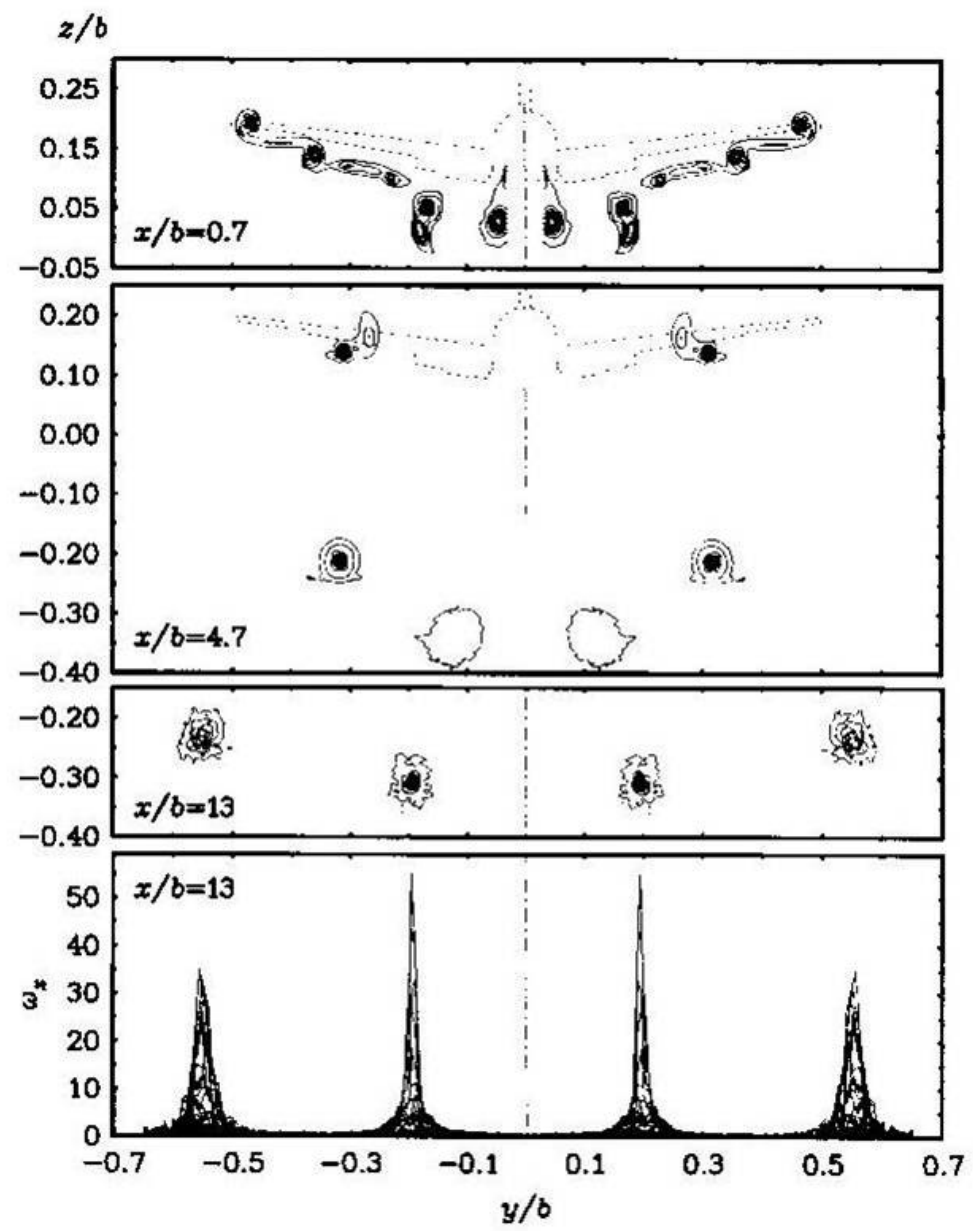

Figure 9.1: Experimental study performed by de Bruin et al. ${ }^{15}$ on a Fokker airliner model showing additional vortex systems.

viscosity acts to make this flow more wake like, and for light loading configurations $\left(\Gamma / U_{\infty} b \ll 1\right.$, where their theory is valid), a deficit in good agreement with experimental data results; the magnitude of the deficit was an order of magnitude less than the freestream. The wake-like profile is a result of the boundary layers that develop over the wing as well as the upstream thrust produced by the axial relaxation of the core pressure caused by radial diffusion of vorticity.

As the loading is increased, the influence of the Bernoulli effect becomes more 
important. This can be seen in the experimental study of Chow et al. ${ }^{13}$ that focused on a relatively high Reynolds number study of a low aspect ratio airfoil at a high angle of attack. The maximum azimuthal velocity of the resulting tip vortex was approximately equal to the free stream velocity and an axial velocity excess (jet) of similar size was observed. The acceleration of the jet-like velocity was accompanied by a measured drop in static pressure over the chord of the wing and almost constant total pressure, consistent with the Bernoulli effect. During the subsequent roll-up of the wake, the static pressure remained relatively constant and the axial velocity weakened slightly, indicating that viscous effects were roughly balanced by the Bernoulli effect in this region. While the swirl parameter for the resulting vortex is approximately 2 , which renders the isolated configuration stable, it is conceivable that higher loading could produce an unstable configuration.

\section{Experimental vs. Numerical configurations}

Experimental studies that investigate the nature of the turbulent vortex, as well as its interaction with co- or counter-rotating pairs, generally take one of two approaches. Analogous to the true aircraft wake, the first approach uses a lifting body to generate vortices, whose spatial development is tracked in the test section once a statistically steady state is reached ${ }^{13}$. The second approach, shown in Figure 9.2, is to generate spatially homogeneous vortices shed from a vortex generator as in the experiments of Leweke and Williamson ${ }^{40}$. The transient evolution of the pair is tracked and compared to the spatially developing wake by replacing t with $x / U_{\infty}$. The key difference between the two is that while three-dimensionality results in the latter from the ensuing instabilities, the base flow is two-dimensional in nature; obviously no mean axial flow results in this case. A more subtle yet related effect is in the axial pressure gradient that develops in the spatially developing wake due to intensification of the vortex during roll-up, and subsequent relaxation as it diffuses; these are transient phenomena in the later experiment and provide no forcing in the axial momentum equation.

From a computational perspective, it is difficult to simulate the full spatial evolu- 


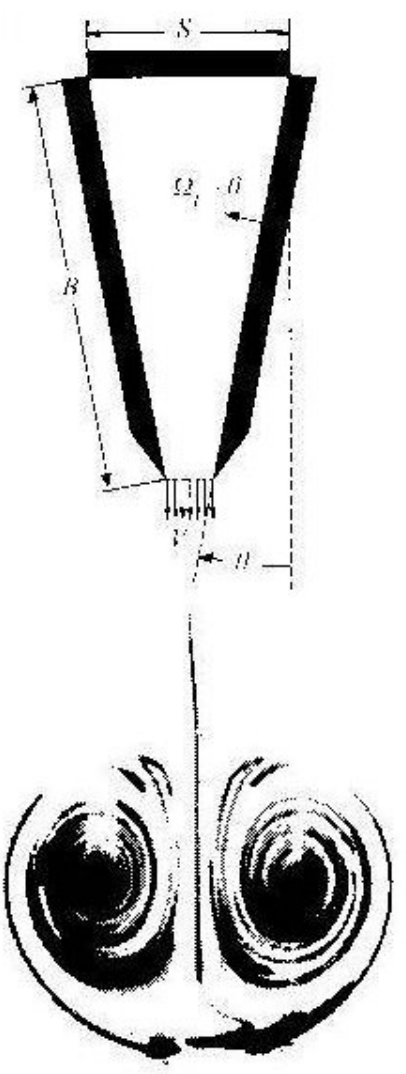

Figure 9.2: Illustration of a device used to generate a counter rotating pair that is essentially 2 dimensional. Figure adapted from $\mathrm{LW}^{40}$.

tion of the vortex and so simulations of the first experimental setup typically employ the temporal approximation as shown schematically in Figure 9.3. This typically has the effect of constraining the base flow to be two-dimensional and hence results in a simulation that is more closely related to the second experimental setup. Sreedhar and Ragab ${ }^{66,67}$ studied the effect of axial flow on a model of an isolated tip vortex using this formulation. They showed that for unstable values of the swirl parameter the vortex becomes turbulent before relaminarizing once the axial velocity had decreased sufficiently. While these simulations were in agreement with the spatial experimental study of Phillips and Graham ${ }^{51}$, the loss of the spatial nature of the flow made it impossible to distinguish between jet- and wake-like axial flow and hence reproduce the subtle differences detected in the experiment. 


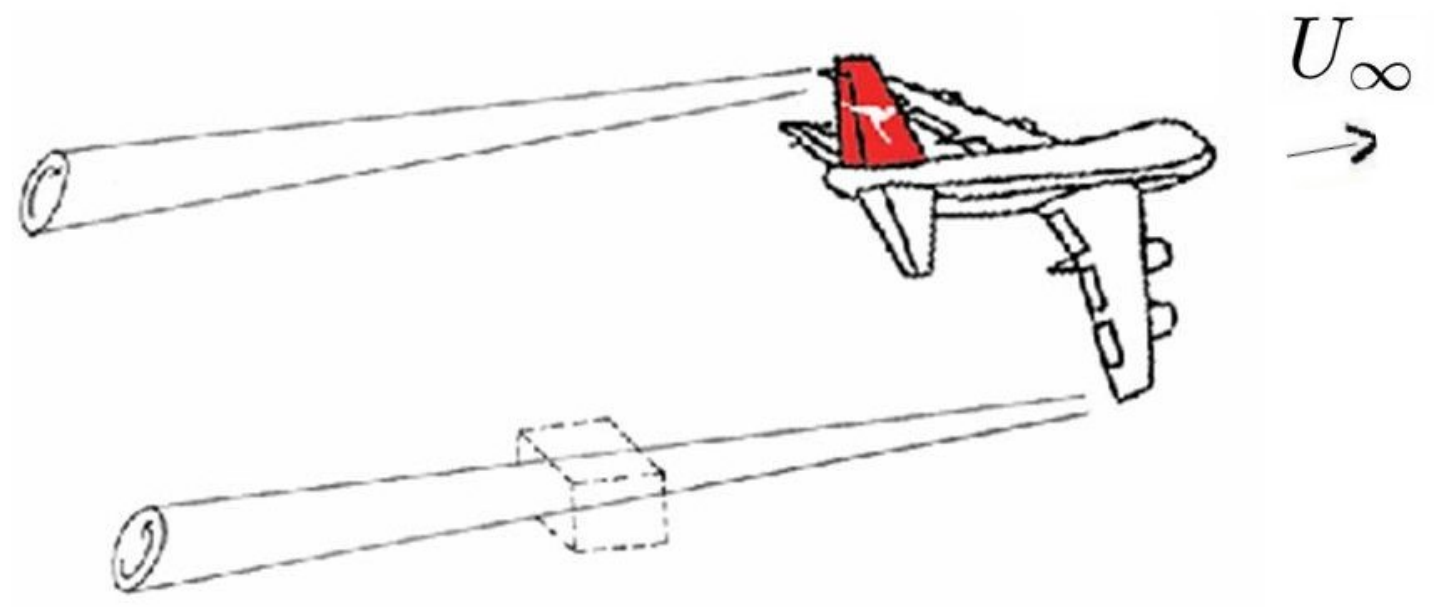

Figure 9.3: Schematic illustration of the temporal approximation. Axial periodicity is enforced on a small domain with a spatial length scale long enough to capture the turbulent motions. Evolution in time of the periodic vortex on this domain is likened to the spatial evolution of the real wake.

In the following section, a model is formulated to incorporate into the temporal simulation, the effect of axial pressure gradients that develop in the spatially developing vortex. It is hoped that by including the Bernoulli effect and retaining information about the flow's spatial nature, we will be able to create a better model of the true three-dimensional wake than is obtained by simply studying three-dimensional disturbances on inherently two-dimensional structures. This allows the distinction to be made between jet- and wake-like axial flow and provides a mechanism to prolong the core turbulence. Subsequently, we investigate how the Bernoulli effect influences the merger of co-rotating pairs and, in particular, the axial velocity that results.

\subsection{Modeling Axial Pressure Gradients}

In the true spatial evolution of a wing tip vortex, an axial pressure gradient develops in the core as the swirling velocity diffuses radially due to both viscous and turbulent diffusion. This pressure gradient produces an axial forcing, as can be seen from the 
axial momentum equation

$$
\frac{\partial u_{1}}{\partial t}+u_{j} \frac{\partial u_{1}}{\partial x_{j}}=-\frac{\partial p}{\partial x_{1}}+\frac{1}{R e} \frac{\partial^{2} u_{1}}{\partial x_{j}^{2}} .
$$

When the temporal approximation is used to replace the infinite axial extent of the physical domain with transient evolution of a finite periodic domain, this phenomenon is also confined to a transient nature, since periodicity prevents any average axial gradients in the velocity components from developing.

$$
\int_{0}^{2 \pi} \frac{\partial u_{i}}{\partial x_{1}} d x_{1}=u_{i}(2 \pi)-u_{i}(0) \equiv 0
$$

Since axial velocity appears to play a critical role in the transition to turbulence of a wing tip vortex ${ }^{64}$, we propose a model for the $x$-average pressure gradient that provides a driving force in the real flow but is absent from the axial momentum equation in the simulation. Such a model is possible in the periodic framework, since only the pressure gradient enters the formulation and not the pressure itself. Hence we can write

$$
\frac{\partial p}{\partial x_{1}}=\frac{\overline{\partial p}}{\partial x_{1}}\left(x_{2}, x_{3}, t\right)+\frac{\partial p^{\prime}}{\partial x_{1}}\left(x_{1}, x_{2}, x_{3}, t\right)
$$

where the first term represents the average pressure gradient that we intend to model and the second term fluctuating pressure gradients with zero mean, $p^{\prime}\left(x_{1}=L\right)=$ $p^{\prime}\left(x_{1}=0\right)$.

It is important to note that the first term must be imposed externally, equivalent to a body force, in a manner similar to as is done in channel flow simulations ${ }^{75}$, i.e., it is not a result of the base flow since all velocity components are axially periodic. We model this term by using the Taylor approximation to replace the mean axial gradient by a temporal derivative included as a source term in the axial momentum equation.

$$
\frac{\overline{\partial p}}{\partial x_{1}}=\frac{1}{L} \int_{0}^{L} \frac{\partial p}{\partial x_{1}} d x_{1} \longrightarrow \frac{1}{L U_{\infty}} \int_{0}^{L} \frac{\partial p}{\partial t} d x_{1}
$$


Hence the axial momentum equation with modeled term reads

$$
\frac{\partial u_{1}}{\partial t}+u_{j} \frac{\partial u_{1}}{\partial x_{j}}=-\frac{1}{L U_{\infty}} \int_{0}^{L} \frac{\partial p}{\partial t} d x_{1}-\frac{\partial p^{\prime}}{\partial x_{1}}+\frac{1}{R e} \frac{\partial^{2} u_{1}}{\partial x_{j}^{2}}
$$

Once again, it should be noted that such a model is not necessary for simulating the experiments of LW since their vortex generator creates a transiently evolving vortex of finite extent similar to what is actually simulated in the code. Before (9.4) can be applied, an assumption must be made about the velocity of the lifting surface, $U_{\infty}$. Assuming lifting line theory and an elliptically loaded airfoil, the lift coefficient $C_{L}$ can be shown ${ }^{46}$ to be

$$
C_{L}=\frac{\pi A_{r} \Gamma_{0}}{2 b U_{\infty}}
$$

where $A_{r}$ is the aspect ratio of the wing of span $b$ with circulation about the central section of the wing $\Gamma_{0}$. Shifting focus now to the trailing vortex pair which is fully rolled up by $\approx \mathcal{O}(10)$ chord lengths, it can be argued from lifting line theory that each has a circulation $\Gamma_{0}$. Further, if these vortices are approximated by Rankine vortices $\left(\Gamma_{0}=2 \pi r_{c} V_{c}\right)$ it can be shown ${ }^{46}$ that

$$
\frac{2 r_{c}}{b} \approx 0.171
$$

Combining these results

$$
U_{\infty}=\frac{0.171 \pi^{2}}{2} \frac{A_{r}}{C_{L}} V_{c}
$$

For typical values of $A_{r}$ and $C_{L}$ (8 and 0.7 respectively), $U_{\infty} \approx 10 V_{c}$, which is similar to the observed values of maximum axial velocity in the experiments of Phillips and Graham $^{51}$. Equations (9.4) and (9.8) in conjunction with an appropriate numerical differencing approximation for the first derivative operator form a tractable model that can be applied to the NS code; the presence of a point at infinity fixes the absolute value of the pressure and hence allows the temporal pressure derivative in (9.4) to be defined in the incompressible framework. It is the retention of physics of the spatial nature of the true flow through this modeled forcing that allows a jet-like 
axial profile to be distinguished from a wake. Imposing the modeled pressure body force as described fixes the positive axial direction as pointing away from the wing; the pressure drop in the core of an isolated vortex, diffusing spatially, causes a force towards the airfoil. A wake-like flow is defined as one in which the axial velocity is in the same direction as this force, while a jet has opposite sense. Finally, the preceding analysis shows that while a typical aircraft flies at around Mach 0.9, this study of the wake is performed in a reference frame where the important velocity scale is an order of magnitude less than the plane speed, and hence the assumption of incompressibility is valid.

\subsection{Effect on Isolated Vortices}

The effect of superimposing jets and wakes on an isolated turbulent vortex was studied by Phillips and Graham ${ }^{51}$. In an attempt to model these experiments, LES were performed at their Reynolds number of 8,536 using the model for axial pressure gradients. The time scale for co-operative instabilities to influence the M\&M initial condition is large compared to that for the axial flow phenomena being investigated and hence the early development of each vortex is considered as if it were isolated. The modeled pressure gradient provides not only a means for distinguishing between the two flows but also a mechanism for altering the rate at which the axial velocity decays and hence the period over which the core of each vortex is unstable to disturbances. Figure 9.4 shows the evolution of $\langle\epsilon\rangle$ for both jet and wake scenarios with $q=1$. While the pressure gradient acts to keep the instability mechanism active longer for the wake-like flow, the dissipation levels are lower than for a jet. If one views the early flow development as characterized by a transfer of energy from the axial flow to turbulent fluctuations in the core, then it is reasonable to assume that the pressure forcing enhances or retards the rate of transfer for jets or wakes respectively. To investigate the differences in the turbulent structure of the vortex for each scenario, the cylindrically-averaged mean flow is first computed and then radial profiles of the fluctuating components of velocity determined. Figure 9.5 shows the resulting profiles 


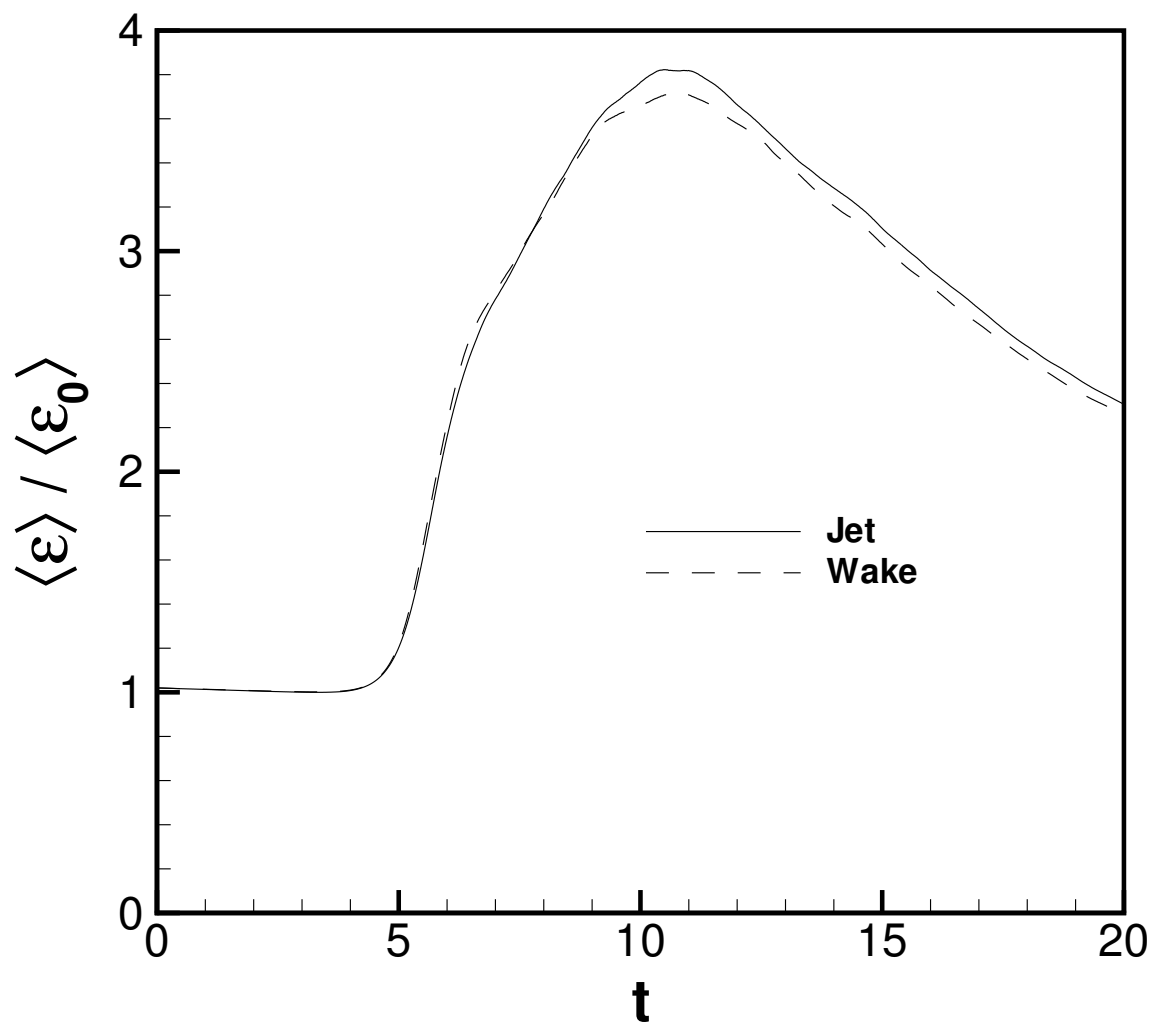

Figure 9.4: Comparison of the evolution of the dissipation, between $q=1$ jet- (solid lines) and wake-like (dashed lines) axial profiles for the LES of the $R e=8,536 \mathrm{M} \& \mathrm{M}$ vortex with $C=0.9$.

at two different instants in time corresponding to $q \approx 1.8$. It can be seen that, in agreement with the experiments ${ }^{51}$, the turbulence quantities attain higher values, extending over greater radii, for jet-like flows than for wake-like flows. It should be noted that a smaller region of the core is considered for the radial and azimuthal components since the flow can only be approximated as cylindrical at small radii.

While the presence of pressure gradients acts to preserve the wake-like velocity profile, hence prolonging the period of helical instability, the axial velocity still decreases and the vortex evolves towards a relaminarized state. Before this state is reached, however, the cooperative instabilities take over, leading to eventual breakdown as discussed in Chapters 7 and 8. While it can be hypothesized that the stronger 
(a)

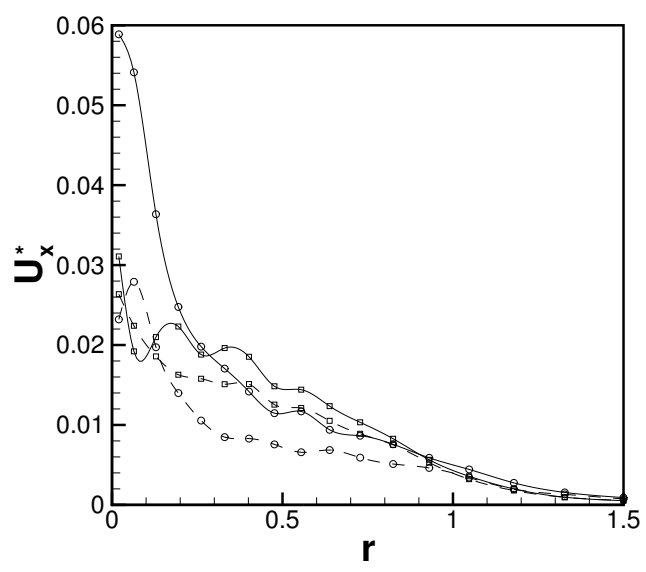

(b)

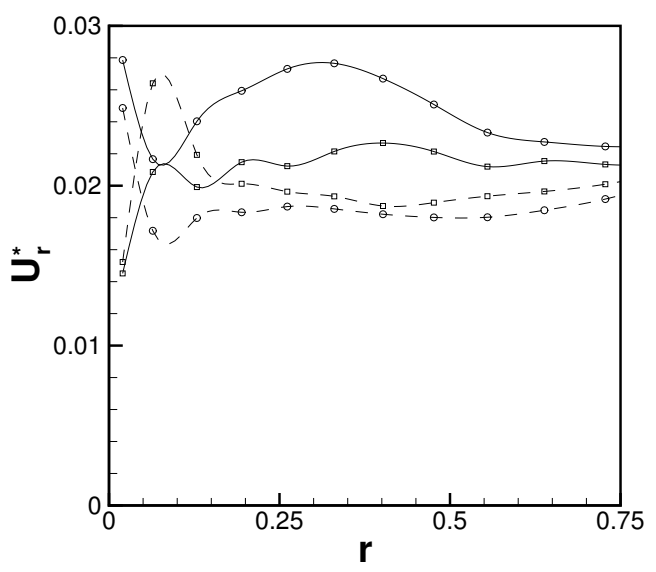

(c)

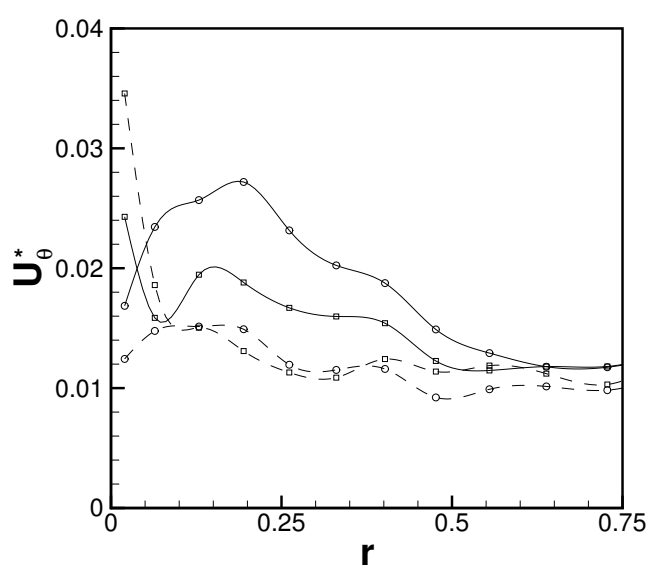

Figure 9.5: Radial distributions of the $\theta$ averaged fluctuating components of (a) axial velocity, (b) radial velocity and (c) azimuthal velocity at $\mathrm{t}=10.8$ (squares) and $\mathrm{t}=15.8$ (circles) for the $q=1, R e=8,536 \mathrm{M} \& \mathrm{M}$ initial condition. Solid lines are for a jet like axial flow and dashed lines for wakes. 
axial velocities resulting for the jet would delay the eventual vortex breakdown (as seen earlier), the meaning of the pressure model becomes unclear once axial homogeneity is lost. Finally, the experiments of Phillips and Graham were conducted at relatively low airfoil loading; the unmodified flow had a wake-like profile in-keeping with Moore and Saffman. Equation 9.8 suggests that these effects would be magnified as the loading is increased. Such a configuration is likely to occur during take off and landing. This phase is of particular interest due to the presence of additional vortex systems resulting from the flap tips, the consequences of which are presented in the following section.

\subsection{Effect on Co-rotating vortex merger}

The merger of co-rotating vortices presents an interesting problem to investigate further, the role that axial pressure gradients play in spatially evolving wakes. Bertenyi ${ }^{4}$ studied this problem experimentally and observed vortex merger significantly sooner than was predicted by two-dimensional simulations. The rotation rate at which the vortices orbit one another increased, causing them to move closer together; the differences were therefore attributed to three-dimensional phenomena. During the take off and landing phase of aircraft, where this problem is of interest, the wing loading is much higher and the swirling velocities in the wake are of the same order as the plane speed. This coupled with the the pressure change during vortex merger leads to a much larger 'modeled' contribution than occurred in the light loading single vortex experiments.

We perform three-dimensional simulations using an initial condition comprising two axisymmetric vorticity distributions, like-signed, and separated by approximately three core radii, similar to as discussed in Section 4.5. On top of this is superposed an axial flow on each vortex with a stable swirl number of $q=1.6$. Image flows associated with the boundary conditions imposed at about six core radii have a non-negligible affect on the flow; however, in line with the previous chapters, these instability mechanisms occur on a much longer time scale than are of interest currently. Figure 9.6 
shows the evolution of the pressure minima for $R e=1000$. Initially, the pressure re-

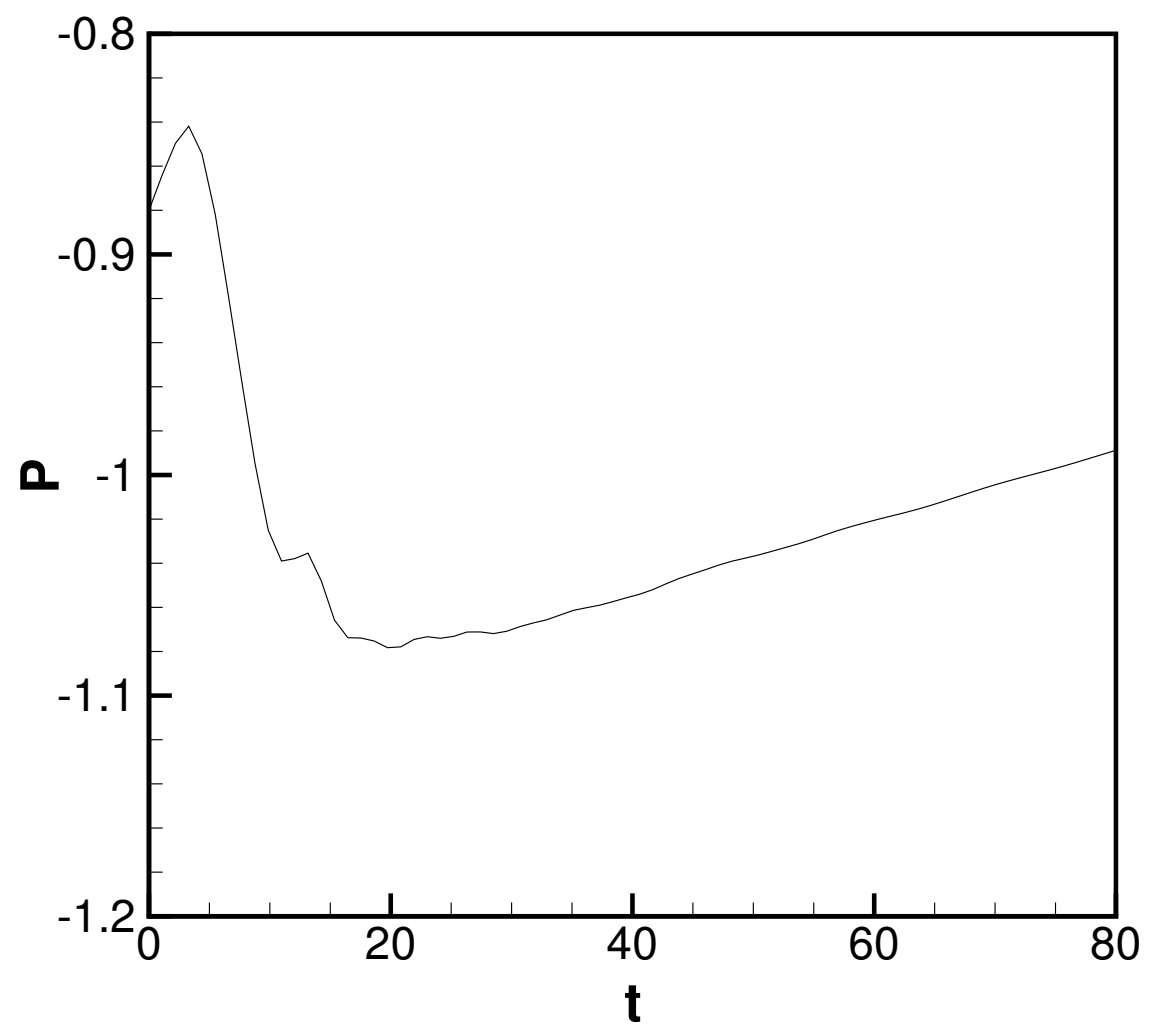

Figure 9.6: Evolution of pressure minima for the temporal merger of co-rotating vortices, $R e=1000$.

laxes as each vortex diffuses; however, merger begins and the pressure drops suddenly as the pair merge into a single larger vortex. Once the cores have merged by about $\mathrm{t}=20$, diffusion once again causes the pressure to relax towards the ambient.

This rapid drop in pressure as the cores merge produces a thrust force that acts to increase the magnitude of the axial jetting. To investigate this effect, the simulation is repeated using the pressure gradient model with $U_{\infty}=U_{\theta_{\max }}$. Figure 9.7 shows a comparison between the two simulations through vorticity contours taken on a slice in the plane of the vortex. In conjunction with Figure 9.9, which shows the evolution of the swirl number, it can be seen that without the modeled pressure gradient the vortices merge in essentially a two-dimensional manner, the axial velocity behaving 
effectively as a passive scalar. In contrast however, the pressure forcing accelerates the axial flow, resulting in a corresponding decrease in the swirl number into the unstable regime. The generation of small scale motions through this instability mechanism ensue and cause the vortex to become turbulent. Once again, the instability mechanism weakens and the vortex relaminarizes as a single vortex at later times.

These simulations identify a mechanism for enhancing the axial flow within trailing vortices to the extent that the vortex can become unstable, resulting in the observed helical instability structure. It is hypothesized that the eventual cooperative instabilities that result from neighboring pairs will be similar to those seen in the previous chapter, whereby the helical structure persists and the onset of the circulation decay is prolonged. This emphasizes the important differences that can result between the spatial evolution and standard temporal approximation. Unfortunately, it seems unlikely that this mechanism alone can explain the faster merger times seen in the experiments, since the axial thrusting does not appear to affect the relative rotation rate between the two vortices. Perhaps this mechanism, coupled with the spatial spiraling of the vortices about one another, could cause the observed result. It is unclear, however, how this effect can be included in the temporal simulation. 

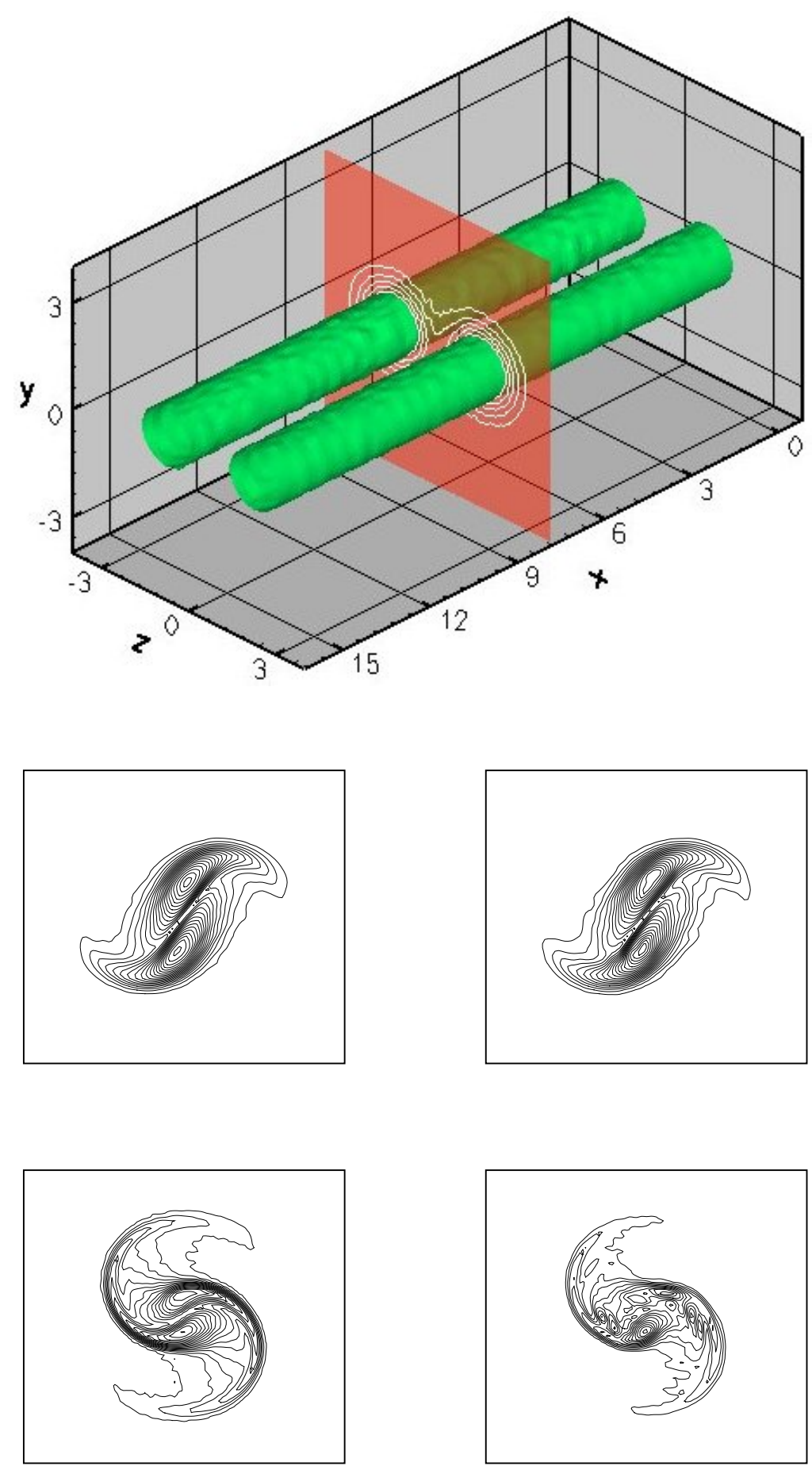

Figure 9.7: Visualization of co-rotating vortex merger through contours of vorticity on a slice in the plane of the vortex. The initial condition is shown (top) with the visualization plane defined. The flow state at $\mathrm{t}=5$ and $\mathrm{t}=10$ is shown both with (right) and without (left) the pressure gradient model activated. $R e=1000$. 

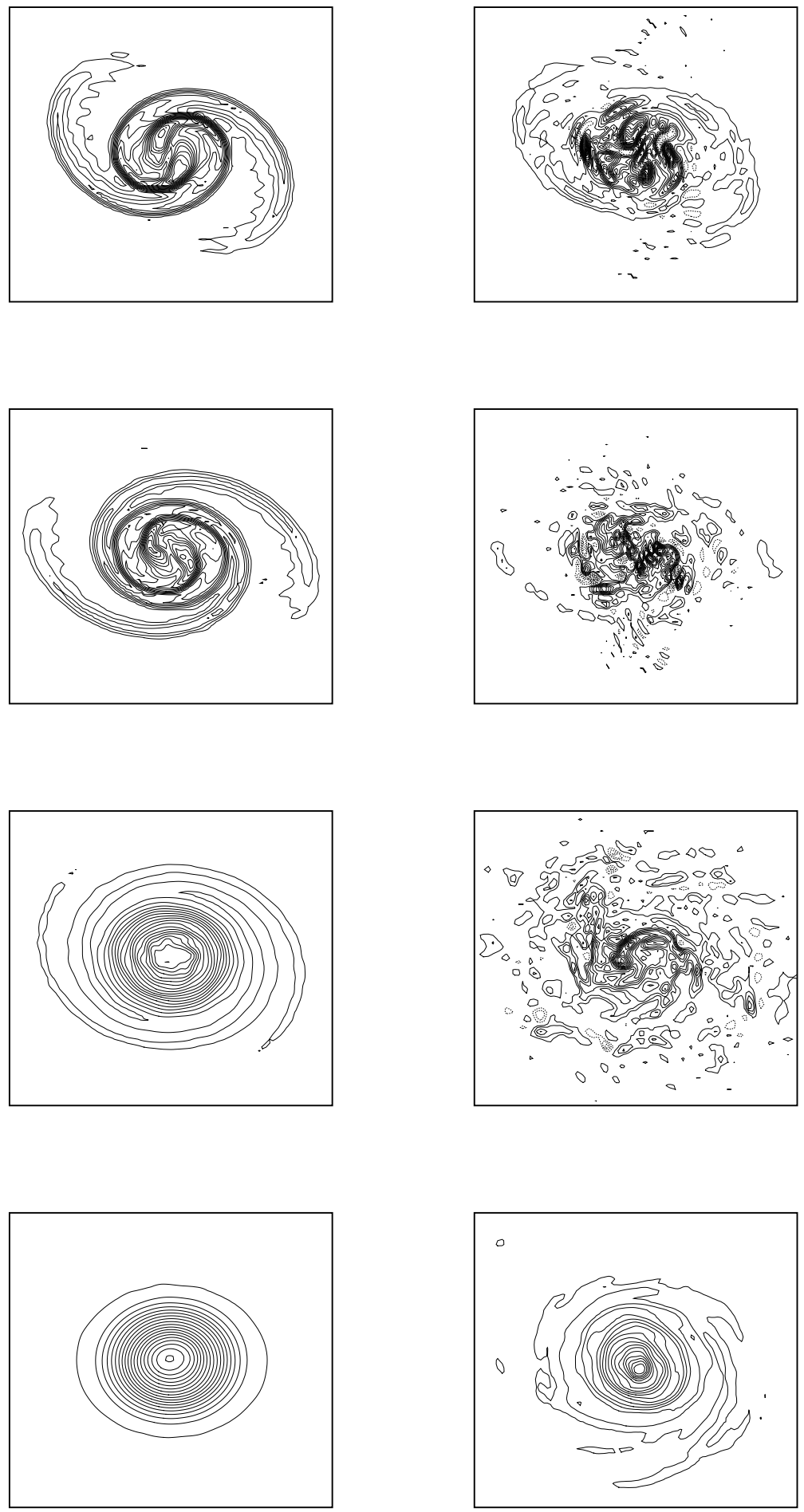

Figure 9.8: Contours of vorticity on the visualization plane defined in Figure 9.7. The flow state is shown at four times corresponding to $\mathrm{t}=15,20,40$ and 80 (top to bottom) both with (right) and without (left) the pressure gradient model activated. $R e=1000$. 

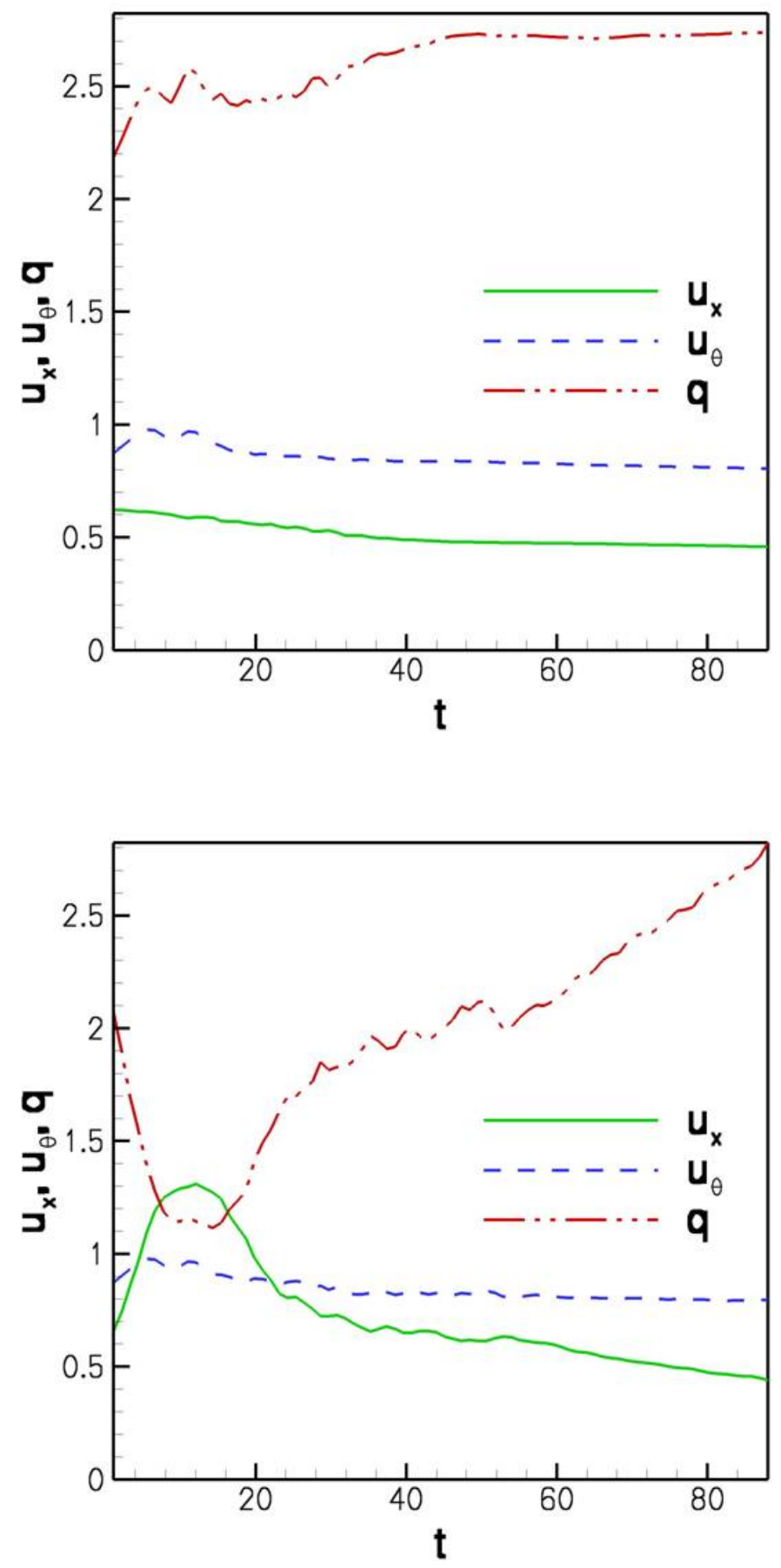

Figure 9.9: Evolution of the maximum swirl and axial velocity component, as well as the swirl parameter $q$, during the merger of co-rotating vortices both with (bottom) and without (top) the model for axial pressure gradients. $R e=1000$. 


\section{Chapter 10}

\section{Concluding Remarks}

This study has focused primarily on a model for the trailing vortex wake comprising an array of counter rotating structures with a superposed axial velocity representative of the roll-up process. The particular form of the initial condition chosen is an exact solution of the 2D-3C steady Euler equations on a domain of infinite extent perpendicular to the plane of the array, thus enabling the linear stability properties to be investigated. We find that, in addition to the slowly growing co-operative elliptic instabilities, the presence of sufficient axial flow leads to modes with significantly higher growth rates. DNS show these modes to be helical in nature and they appear confined to each structure independent of the array. In agreement with theoretical predictions and previous DNS of a model of an isolated trailing vortex, the turbulence generated as a result of these modes decays. This leads eventually to relaminarization owing to a reduction in the axial flow and hence the underlying instability mechanism. At later times the co-operative instabilities take over. The periodic analogue of the Crow instability pulls portions of each pair together where vortex breakdown is initiated via mixing caused by the short-wavelength Widnall modes. Despite causing higher initially linear growth rates, the presence of axial flow results in structures that appear to resist this break-down phenomenon for longer periods than for vortices without axial flow. Analysis of the PDF's of alignment between the vorticity and the eigenvalues of the rate of strain tensor show an evolution from an initially organized state towards a final state in which preferential alignment with the intermediate 
eigenvector is observed. This is similar to what is seen in isotropic turbulence and is further evidence that vortex-disintegration has occurred rather than the reconnection expected in the absence of the Widnall modes.

These trends were investigated at higher Reynolds numbers by LES using the stretched vortex subgrid model. The presence of large scale rotation and an infinite domain provided a challenging flow regime to test the physical-space version of the model beyond the benchmark test cases of box turbulence and channel flow. Once again, two fundamentally different flow regions are identified; however, while the turbulence in each vortex, generated by the fast axial velocity related instabilities, decreases significantly, relaminarization is not observed. Presumably, significant growth of the co-operative modes occurs before the lower viscosity has damped out the weakening helical modes. This configuration results in delaying the disintegration of the vortex longer than at lower Reynolds numbers. By comparing the dissipation, nondimensionalized by the vortex core radius and velocity at different Reynolds numbers, a mixing transition was identified at $R e=1-2 \times 10^{4}$. This indicates that beyond this threshold the flow is less influenced by Reynolds number; an idea supported by flow visualization. Future work as computational resources improve would be to compute a DNS at $\mathrm{Re}=20,000$, hence providing the basis for a comprehensive understanding of the behavior of the true aircraft wake at higher Reynolds numbers.

Finally, we considered the differences between studying three-dimensional instabilities on two-dimensional vortices, as is typically done numerically, and the real three-dimensional spatial evolution of the airfoil wake. One difference between the two is that imposing axial periodicity discretizes the spectrum of supportable axial instability modes. The current simulations however, appear relatively insensitive to this as long as the most unstable Crow mode is captured; the role of the long modes appears to be predominantly associated with pulling neighboring vortices together where mixing occurs via the short wavelength instability. While only a couple of such long modes can be captured, many small wavelength modes exist to describe the non-linear interactions at this scale. A more subtle difference is in the axial pressure gradient that develops as the spatial vortex diffuses radially. A model for 
this phenomenon was formulated, and hence aspects of the spatial nature of the real flow incorporated into the simulation. This made it possible to distinguish between wake-like and jet-like axial flow and also provide a mechanism to enhance the period of turbulence within the vortex core. For light loading configurations it was found, in agreement with Phillips and Graham ${ }^{51}$, that a jet resulted in higher turbulence intensities. Pressure gradients were not strong enough to sustain turbulence, and relaminarization of the isolated vortex can be predicted in further agreement with the experiments.

For light loading conditions, a wake-like axial velocity profile with magnitude below that required to render the isolated vortex unstable is typical. During the take-off and landing phase however, the higher loading results in swirling velocities of similar magnitude to the plane velocity. The associated drop in pressure can cause strong jet-like axial flow to be present. While experiments at high angles of attack show that the axial velocity is still too low to cause the isolated instabilities, it is possible that merger of the co-rotating systems resulting from flap tips can cause a further drop in a pressure and hence increased axial flow. Such a case was considered and highlights the short-comings of the unmodified temporal approximation whereby the axial velocity behaves essentially as a passive scalar mixed by the two-dimensional base flow. Incorporating the model for axial pressure gradients causes the axial flow to strengthen during the merger process and three-dimensional instabilities to develop. The characteristic weakening of the instability mechanism occurs at later times, causing the merged vortex to relaminarize. While the model allows some aspects of the spatial nature of the wake to be realized in the temporal framework, it appears that the spatial spiraling of the vortices about each other must be taken into account for accurate 'time-to-merger' predictions to be made. For such a prediction the constraints imposed by the temporal approach seem inescapable and the full spatial evolution needs be simulated.

The main findings of this research can then be summarized as:

- Superposition of a sufficiently strong axial flow on the M \& M vortex array causes instability modes that develop independently on each vortex in the ar- 
ray, the associated growth rates being much stronger than for the cooperative instabilities.

- The modes associated with these instabilities are helical in nature, and extract energy from the axial flow.

- The axial flow decreases, weakening the instability mechanism to the point where relaminarization can be observed before the cooperative modes take over.

- At higher Reynolds numbers (presumably also for smaller values of the separation parameter $\mathrm{C}$ ), the cooperative instabilities take over before relaminarization occurs.

- A mixing transition for this initial phase is observed at around $R e=20,000$.

- The helical nature of the initial instability persists and retards mixing via the short wavelength cooperative modes.

- The effect of axial pressure gradients consequent of a spatially decaying line vortex can be modeled in the framework of the temporal approximation, allowing jet- and wake-like axial flows to be distinguished. Higher turbulence intensities result from the former in accordance with previous experimental work ${ }^{51}$.

- Axial pressure gradients provide a mechanism for enhancing the axial-flow related instabilities to the point where a pair of co-rotating vortices, each initially stable with respect to this mechanism, merge in a three-dimensional manner. 


\section{Appendix A}

\section{Point Vortex Induced Strain}

A derivation of the strain at the center of a point vortex, induced from either a single counter rotating point vortex or an array of counter rotating point vortices is presented to examine the effect of image flow arising from imposing periodic boundary conditions. The complex potential for flow in the plane of a line vortex of negative orientation at the point $(\mathrm{x}, \mathrm{y})=(\mathrm{a}, 0)$ is given by

$$
F_{p t}(z)=i \frac{\Gamma}{2 \pi} \log (z-a)
$$

from which the complex velocity is computed as

$$
W_{p t}(z)=u-i v=\frac{d F}{d z}=i \frac{\Gamma}{2 \pi z}
$$

To determine the strain induced at the center of a counter rotating neighbor located at the origin, consider the rate of strain tensor $S_{i j}$ given in matrix form as

$$
\left(\begin{array}{cc}
0 & \frac{\Gamma}{2 a^{2} \pi} \\
\frac{\Gamma}{2 a^{2} \pi} & 0
\end{array}\right)
$$

This has orthogonal eigenvectors at \pm 45 degrees to the $\mathrm{x}$ axis with corresponding

eigenvalues $\mp \frac{\Gamma}{2 a^{2} \pi}$. Now consider an array of co-rotating line vortices of $\mathrm{x}$ period $2 \mathrm{a}$, 
having complex potential

$$
F_{c o}(z)=-i \frac{\Gamma}{2 \pi} \log \left(\sin \left(\frac{\pi z}{2 a}\right)\right)
$$

We form the counter rotating array by superposing an opposite sign array offset by distance a.

$$
F_{c r}(z)=-i \frac{\Gamma}{2 \pi} \log \left(\sin \left(\frac{\pi z}{2 a}\right)\right)+i \frac{\Gamma}{2 \pi} \log \left(\sin \left(\frac{\pi(z-a)}{2 a}\right)\right)
$$

To determine, the strain induced by the array at the center of the vortex located at the origin, the point vortex complex potential (of positive sense) is subtracted and the complex velocity evaluated as

$$
W_{c r}(z)=i \frac{\Gamma}{2 \pi z}-i \frac{\Gamma}{4 a} \cot \left(\frac{\pi z}{2 a}\right)+i \frac{\Gamma}{4 a} \cot \left(\frac{\pi(z-a)}{2 a}\right) .
$$

This is expanded about the origin and the leading order computed as

$$
W_{c r}(z)=-i \frac{\Gamma \pi z}{12 a^{2}}+O\left[z^{2}\right]
$$

with the associated rate of strain tensor

$$
\left(\begin{array}{cc}
0 & \frac{\Gamma \pi}{12 a^{2}} \\
\frac{\Gamma \pi z}{12 a^{2}} & 0
\end{array}\right)
$$

Once again the eigenvectors are at \pm 45 degrees to the $\mathrm{x}$ axis, however, this time the magnitude of the strain is about $65 \%$ higher with eigenvalues $\mp \frac{\Gamma \pi z}{12 a^{2}}$.

Switching now to the M\&M vortex array, $S_{i j}$ can be evaluated at the origin from the dimensional version of 5.5 with period 2 a giving

$$
\left(\begin{array}{cc}
0 & \frac{\Gamma \pi C}{4 a^{2}} \\
\frac{\Gamma \pi C}{4 a^{2}} & 0
\end{array}\right)
$$

with the same eigenvectors as before, and eigenvalues $\mp \frac{\Gamma \pi C}{4 a^{2}}$. This however, is the total 
strain, included that associated with the vortex at located at the origin. An isolated point vortex has no self induced strain, however, Moore and Saffman ${ }^{49}$ calculated that an exterior imposed strain distorts the Rankine vortex into an ellipse, resulting in a total strain at the origin for the pair of

$$
\frac{\Gamma}{\pi a^{2}} .
$$

Finally, the ratio of the M\&M strain to the vortex pair total strain is calculated as

$$
\frac{c \pi^{2}}{4} .
$$




\section{Appendix B}

\section{Coefficients for Time Integration}

The coefficients $\alpha, \beta$ and $\gamma$ of the stiffly stable integration scheme used for the time integration of N-S as presented in section 4.4.1 are given in the table below. These can be derived formally using Taylor series expansions as discussed in Karniadakis et $a l .{ }^{30}$

Table B.1: Coefficients for stiffly-stable schemes

\begin{tabular}{cccc}
\hline \hline Coefficient & 1st order & 2nd order & 3rd order \\
\hline \hline$\gamma_{0}$ & 1 & $3 / 2$ & $11 / 6$ \\
\hline$\alpha_{0}$ & 1 & 2 & 3 \\
$\alpha_{1}$ & 0 & $-1 / 2$ & $-3 / 2$ \\
$\alpha_{2}$ & 0 & 0 & $1 / 3$ \\
\hline$\beta_{0}$ & 1 & 2 & 3 \\
$\beta_{1}$ & 0 & -1 & -3 \\
$\beta_{2}$ & 0 & 0 & 1 \\
\hline \hline
\end{tabular}




\section{Bibliography}

[1] Wm. T. Ashurst, A. R. Kerstein, R. M. Kerr, and C. H. Gibson. Alignment of vorticity and scalar gradient with strain rate in simulated navier-stokes turbulence. Phys. Fluids, 30(8):2343-2353, 1987.

[2] E. J. Avital, N. D. Sandham, and K. H. Luo. Stretched cartesian grids for solution of the incompressible navier-stokes equations. International journal for numerical methods in fluids, 33:897-918, 2000.

[3] B. J. Bayly. Three-dimensional instability of elliptic flow. Phys. Rev. Lett., 57:2160-2163, 1986.

[4] T. Bertenyi. Merger of Aircraft Wake Vortices. Ph.D. thesis, University of Cambridge, 2001.

[5] J. P. Boris, F. F. Grinstein, E. S. Oran, and R. L. Kolbe. New insights into large eddy simulation. Fluid Dyn. Res., 10:199-228, 1992.

[6] G. Bosch and W. Rodi. Simulations of vortex shedding past a square cylinder with different turbulence models. Int. J. Numer. Meth. Fluids, 28:610, 1998.

[7] R. Breidenthal. Structure in turbulent mixing layers and wakes using a chemical reaction. J. Fluid Mech., 109:1-24, 1981.

[8] J. C. Buell. A hybrid numerical method for three-dimensional spatiallydeveloping free-shear flows. Journal of computational physics, 95:313-338, 1991.

[9] J. D. Buntine and D. I. Pullin. Merger and cancellation of strained vortices. J. Fluid Mech., 205:263-295, 1989. 
[10] A. B. Cain, J. H. Ferziger, and W. C. Reynolds. Discrete orthogonal function expansions for non-uniform grids using the fast fourier transform. J. Comp. Phys., 56:272-286, 1984.

[11] C. Canuto, M. Y. Hussaini, A. Quarteroni, and T. A. Zang. Spectral Methods in Fluid Dynamics. Springer-Verlag, New York, 1988.

[12] M. S. Chong, A. E. Perry, and B. J. Cantwell. A general classification of threedimensional flow fields. Phys. Fluids. A, 2:765-777, 1990.

[13] J. S. Chow, G. G. Zilliac, and P. B. Bradshaw. Mean and turbulent measurements in the near field of a wingtip vortex. AIAA J., 35(10):1561-1567, 1997.

[14] S. C. Crow. Stability theory for a pair of trailing vortices. AIAA J., 8(12):21722179, 1970.

[15] A. C. de Bruin, S. H. Hegen, P. B. Rohne, and P. R. Spalart. Flow field survey in trailing vortex system behind a civil aircraft model at high lift. In Presented at Advis. Group Aerosp. Res. Dev. (AGARD) Symp., Trondheim, Norway, volume 25, pages 1-12, 1996.

[16] P. E. Dimotakis. The mixing transition in turbulent flows. J. Fluid Mech., 409:69-98, 2000.

[17] P. A. Durbin. Separated flow computations using the $k-\epsilon-\nu^{2}$ model. AIAA J., 33(4):659, 1995.

[18] M. Frigo and S. G. Johnson. Fftw: An adaptive software architecture for the fft. In ICASSP conference proceedings, volume 3, pages 1381-1384, 1998.

[19] M. Germano, U. Piomelli, P. Moin, and W. H. Cabot. A dynamic subgrid-scale eddy viscosity model. Phys. Fluids A, 3(7, 12(E)):1760-1765, 3128(E), 1991.

[20] S. Ghosal, T. S. Lund, P. Moin, and K. Akselvoll. A dynamic localization model for large-eddy simulation of turbulent flows. J. Fluid Mech., 286:229-255, 1995. 
[21] S. Girimaji. Partially averaged navier-stokes (pans) method for turbulence: A rans to dns bridging model. In GALCIT fluid mechanics seminar series, Jan 7 2005.

[22] S. P. Govindaraju and P. G. Saffman. Flow in a turbulent trailing vortex. Phys. Fluids, 14(10):2074-2080, 1971.

[23] E. R. Hoffman and P. N. Joubert. Turbulent line vortices. J. Fluid Mech, 16:395$411,1963$.

[24] J. C. R. Hunt, A. A. Wray, and P. Moin. Eddies, stream, and convergence zones in turbulent flows. In Center for turbulence research report CTR-S88, page 193, 1988.

[25] L. Jacquin and C. Pantano. On the persistence of trailing vortices. J. Fluid Mech, 471:159-168, 2002.

[26] J. Jeong and F. Hussain. On the identification of a vortex. J. Fluid Mech., 285:69-94, 1995.

[27] J. Jimenez, A. A. Wray, P. G. Saffman, and R. S. Rogallo. The structure of intense vorticity in isotropic turbulence. J. Fluid Mech., 255:65-90, 1993.

[28] S. Julien, J.-M. Chomaz, and J.-C. Lasheras. Three-dimensional stability of periodic arrays of counter-rotating vortices. Phys. Fluids, 14(2):732-743, 2002.

[29] Y. Kaneda, T. Ishihara, M. Yokokawa, K. Itakura, and A. Uno. Energy dissipation rate and energy spectrum in high-resolution direct numerical simulations of turbulence in a periodic box. Phys. Fluids, 15:L21-L24, 2003.

[30] G. E. Karniadakis, M. Israeli, and S. A. Orszag. High-order splitting methods for the incompressible navier-stokes equations. J. Comp. Phys., 97:414-443, 1991.

[31] Kelvin. On the vibrations of a columnar vortex. Phil. Mag., 5(10):155, 1880. 
[32] R. M. Kerr. Higher-order derivative correlations and the alignment of small-scale structures in isotropic numerical turbulence. J. Fluid Mech., 153:31, 1985.

[33] S. Kida and H. Miura. Identification and analysis of vortical structures. Eur. J. Mech. B/Fuids, 17(4):471-488, 1998.

[34] J. H. Konrad. An experimental investigation of mixing in two-dimensional turbulent shear flows with applications to diffusion-limited chemical reactions. Ph.D. thesis, Caltech, 1977.

[35] B. Kosovic, D. I. Pullin, and R. Samtaney. Subgrid-scale modeling for large-eddy simulations of compressible turbulence. Phys. Fluids, 14(4):1511-1522, 2002.

[36] S. Leibovich and K. Stewartson. A sufficient condition for the instability of columnar vortices. J. Fluid Mech, 126:335-356, 1983.

[37] A. Leonard. Energy cascade in large-eddy simulations of turbulent fluid flows. In F. N. Frankiel and R. E. Munn, editors, Advances in Geophysics, volume 18A, pages 237-249. Academic, 1974.

[38] A. Leonard and A. Wray. A new numerical method for the simulation of threedimensional flow in a pipe. Technical memorandum 84267, NASA, 1982.

[39] M. Lesieur and O. Metais. New trends in large-eddy simulations of turbulence. Annu. Rev. Fluid Mech., 28:45-82, 1996.

[40] T. Leweke and C. H. K. Williamson. Cooperative elliptic instability of a vortex pair. J. Fluid Mech, 360:85-119, 1998.

[41] H. J. Lugt. Vortex flows in nature and technology. John Wiley \& Sons, Inc., 1983.

[42] T. S. Lundgren. Strained spiral vortex model for turbulent fine structure. Phys. Fluids., 25(12):2193-2023, 1982. 
[43] R. Mallier and S. A. Maslowe. A row of counter-rotating vortices. Phys. Fluids A, 5(4):1074-1075, 1993.

[44] T. Matsushima and P. S. Marcus. A spectral method for polar coordinates. Journal of computational physics, 120:365-374, 1995.

[45] T. Matsushima and P. S. Marcus. A spectral method for unbounded domains. Journal of computational physics, 137:321-345, 1997.

[46] L.M. Milne-Thomson. Theoretical Aerodynamics. New York, Dover Publications Inc, 1958.

[47] A. Misra and D. I. Pullin. A vortex-based subgrid stress model for large-eddy simulation. Phys. Fluids, 9(8):2443-2454, 1997.

[48] D. W. Moore and P. G. Saffman. Axial flow in laminar trailing vortices. R. Soc. Lond. A., 333:491-508, 1973.

[49] D. W. Moore and P.G. Saffman. Structure of a line vortex in an imposed strain. In Aircraft Wake Turbulence, pages 339-354. Plenum, 1971.

[50] W. R. C. Phillips. The turbulent trailing vortex during roll-up. J. Fluid Mech., 105:451-467, 1981.

[51] W. R. C. Phillips and J. A. H Graham. Reynolds-stress measurements in a turbulent trailing vortex. J. Fluid Mech, 147:353-371, 1984.

[52] R. T. Pierrehumbert. Universal short-wave instability of two-dimensional eddies in an inviscid fluid. Phys. Rev. Lett., 57:2157-2159, 1986.

[53] S. B. Pope. Ten questions converning the large-eddy simulation of turbulent flows. New J. Phys., 6(35):1-24, 2004.

[54] D. S. Pradeep and F. Hussain. Core dynamics of a strained vortex: instability and transition. J. Fluid Mech., 447:247-285, 2001. 
[55] D. I. Pullin and P. G. Saffman. Reynolds stresses and one-dimensional spectra for a vortex model of homogenous anisotropic turbulence. Phys. Fluids, 6(5):17871796, 1994.

[56] J. H. Qin. Numerical simulation of a turbulent axial vortex. Ph.D. thesis, Purdue university, 1998.

[57] O. Reynolds. On the dynamical theory of incompressible viscous fluids and the determination of the criterion. Phil. Trans. R. Soc. A, 186:123, 1895.

[58] W. C. Reynolds. The potential and limitations of direct and large eddy simulations. In J. L. Lumley, editor, Wither Turbulence? Turbulence at the Crossroads (Lecture notes in Physics vol 357, page 313. Berline: Springer, 1990.

[59] A. C. Robinson and P. G. Saffman. Stability and structure of stretched vortices. Stud. Appl. Maths, 70:163-181, 1984.

[60] P. G. Saffman. A model of vortex reconnection. J. Fluid Mech., 212:395-402, 1990.

[61] P. Sagaut. Large eddy simulation for incompressible flows. Springer, 2002.

[62] E. D. Siggia. Numerical study of small scale intermittency in three-dimensional turbulence. J. Fluid Mech., 107:375-406, 1981.

[63] J. Smagorinksy. General circulation experiments with primitives equations. Mon. Weather Rev., 91:99-164, 1963.

[64] P. R. Spalart. Airplane trailing vortices. Annu. Rev. Fluid Mech., 30:107-138, 1998.

[65] P. R. Spalart. Strategies for turbulence modelling and simulations. Int. J. Heat and Fluid Flow, 21:252-263, 2000.

[66] M. Sreedhar and S. Ragab. Large eddy simulation of longitudinal stationary vortices. Phys. Fluids, 6(7):2501-2514, 1994. 
[67] M. Sreedhar and S. Ragab. Numerical simulation of vortices with axial velocity deficits. Phys. Fluids, 7(3):549-558, 1995.

[68] K. R. Sreenivasan. An update on the energy dissipation rate in isotropic turbulence. Phys. Fluids., 10(2):528-529, 1998.

[69] Katepalli R. Sreenivasan. On the scaling of the turbulent energy dissipation rate. Phys. Fluids., 27:867, 1984.

[70] J. T. Stuart. On finite amplitude oscillations in laminar mixing layers. J. Fluid Mech., 29:417-440, 1967.

[71] B. Tao, J. Katz, and C. Meneveau. Geometry and scale relationships in high reynolds number turbulence determined from three-dimensional holographic velocimetry. Phys. Fluids., 12(5):941-944, 2000.

[72] G. I. Taylor. Statistical theory of turbulence. Proc. R. Soc. London Ser. A, 151:421, 1935.

[73] H. Tennekes and J. L. Lumley. A first course in turbulence. MIT Press, Cambridge, Mass., 1972.

[74] T. Veolkl, D. I. Pullin, and D. C. Chan. A physical-space version of the stretchedvortex subgrid-stress model for large-eddy simulation. Phys. Fluids., 12(7):1810$1825,2000$.

[75] T. Voelkl. A physical-space version of the stretched-vortex subgrid-stress model for large-eddy simulation of incompressible flow. Ph.D. thesis, California Insititute of Technology, 2000.

[76] S. E. Widnall, D. B. Bliss, and C. Y. Tsai. Instability of short waves on a vortex ring. J. Fluid Mech, 66:35, 1974. 